\title{
The influence of common infections on clinical course and neurodegeneration in an animal model of Multiple Sclerosis
}

\author{
Dissertation \\ For the award of the degree \\ "Doctor rerum naturalium" (Dr. rer. nat.) \\ Division of Mathematics and Natural Sciences \\ of the Georg-August-Universität Göttingen
}

Submitted by

Prateek Kumar

From Purnia (India)

Göttingen, 2014 


\section{Thesis committee members}

\section{Prof. Dr. Mathias Bähr}

(1st Reviewer, advisor and member of the thesis committee)

Dept. of Neurology, University Medical Center,

Georg August University of Göttingen

Prof. Dr. Mikael Simons

(2nd Reviewer)

Cellular Neuroscience, Max Planck Institute of Experimental Medicine,

Department of Neurology, Georg August University of Göttingen

Prof. Dr. Klaus-Armin Nave

Department of Neurogenetics, Max Planck Institute of Experimental Medicine, Georg August University of Göttingen

\section{Extended thesis committee members}

Prof. Dr. Wolfgang Brück

Prof. Dr. Tiago Fleming Outeiro

Dr. Camin Dean 


\section{Dedicated to my mother...!}




\section{Declaration}

I hereby declare that the thesis "The influence of common infections on clinical course and neurodegeneration in an animal model of Multiple Sclerosis" has been written independently with no other sources and aids otherwise quoted.

Prateek Kumar,

Göttingen, June 2014. 


\section{Table of Contents}

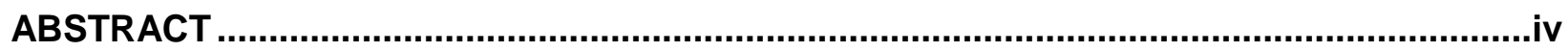

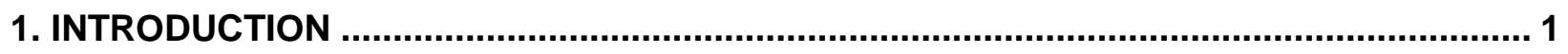

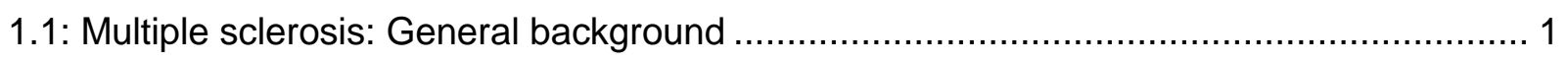

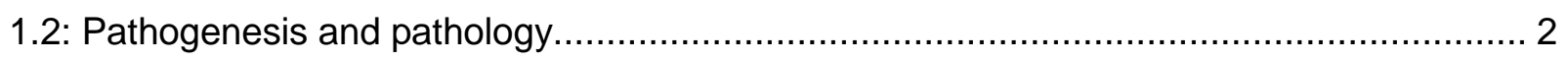

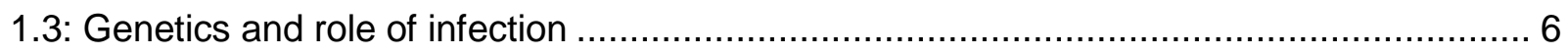

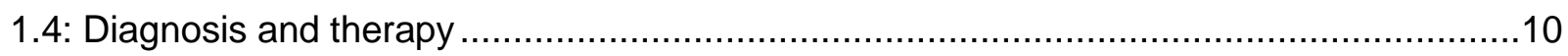

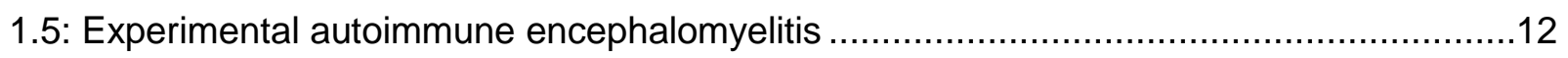

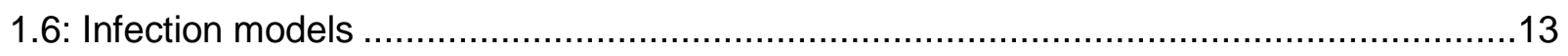

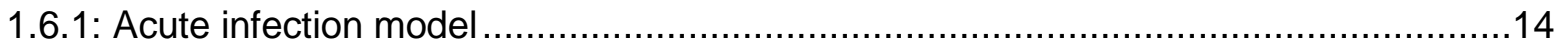

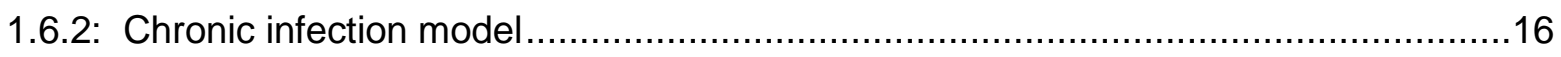

2. AIM OF THIS WORK

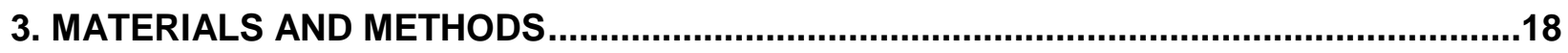

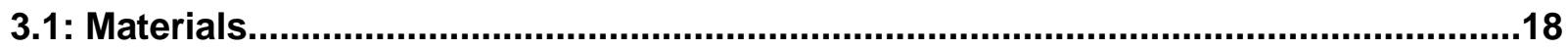

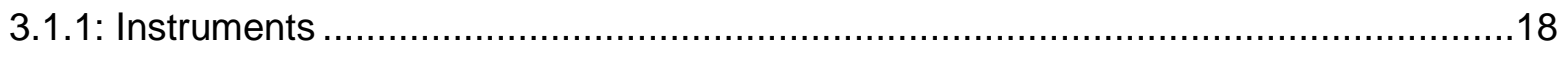

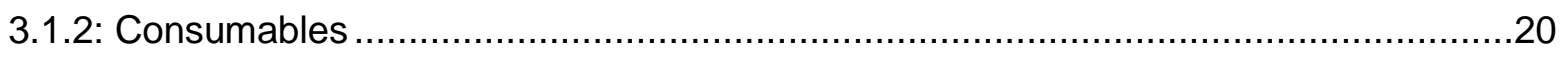

3.1.3: Chemicals, miscellaneous reagents and commercial kits. ..................................21

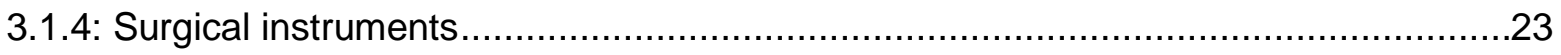

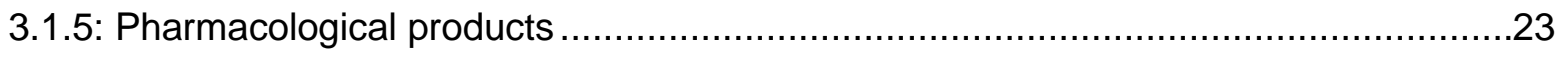

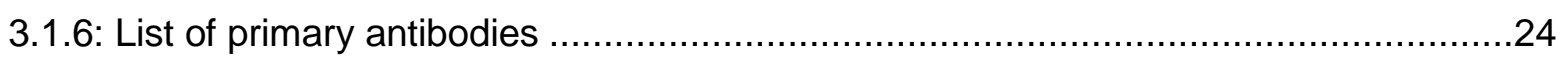

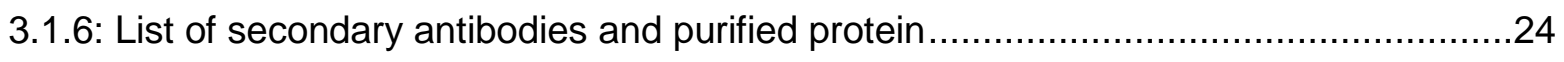

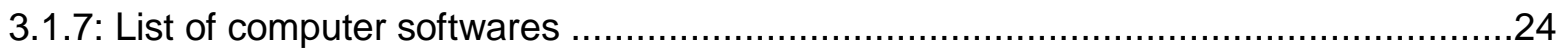

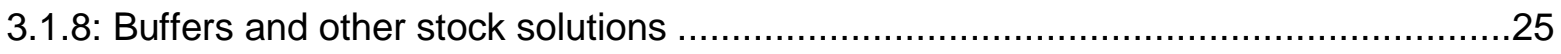

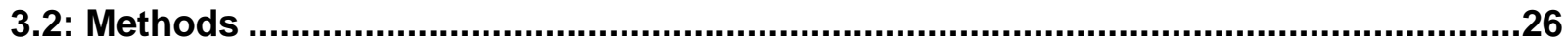

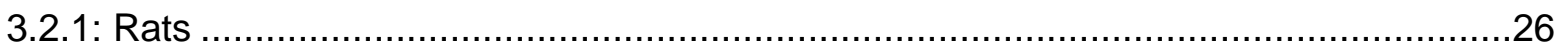

3.2.2: Immunogen

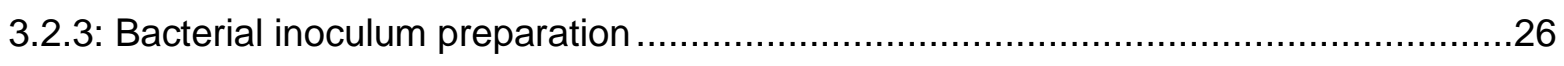

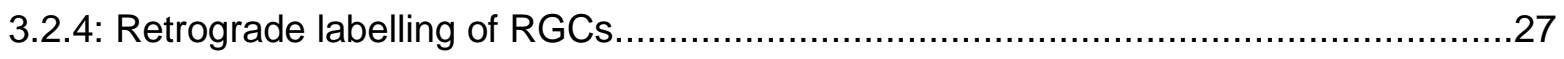




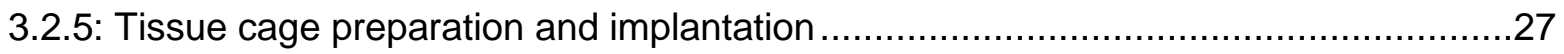

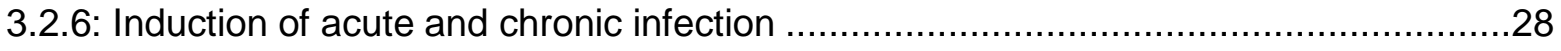

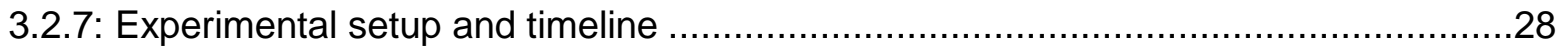

3.2.7.2: Experimental design for chronic infection model and timeline ..........................30

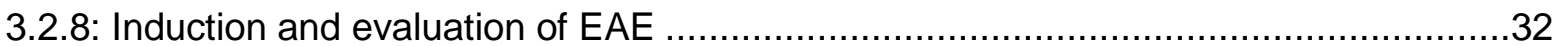

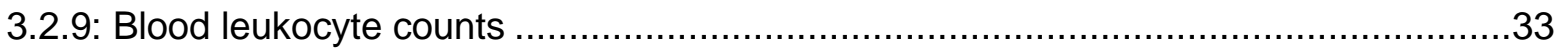

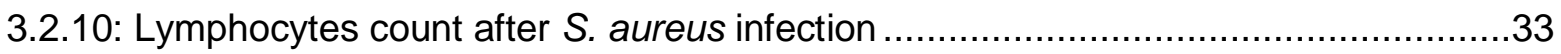

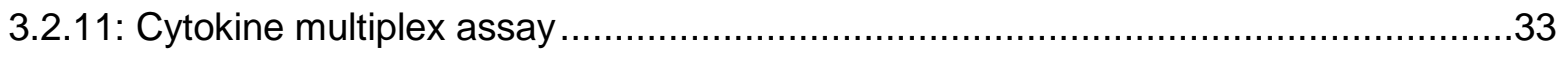

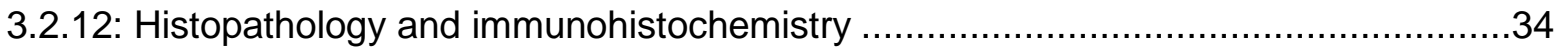

3.2.12.1: Staining and quantification of demyelination ............................................. 34

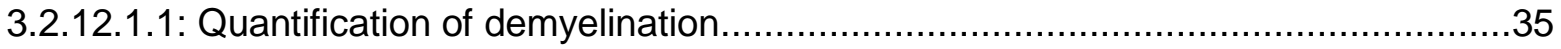

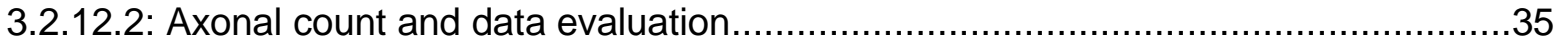

3.2.12.2.1: Quantification of axonal densities in optic nerve ....................................36

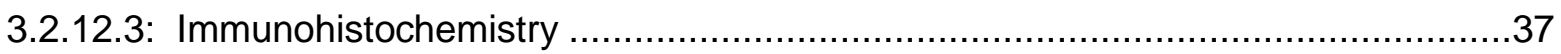

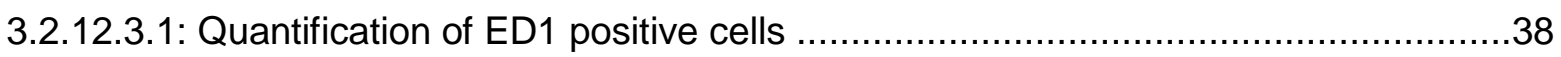

3.2.12.3.2: Quantification of T-cell infiltrates in the optic nerve ..................................38

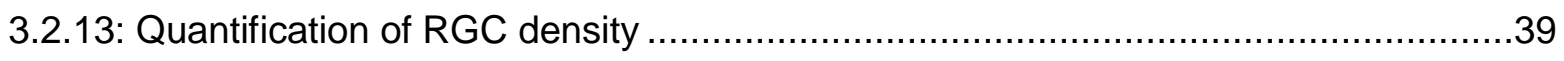

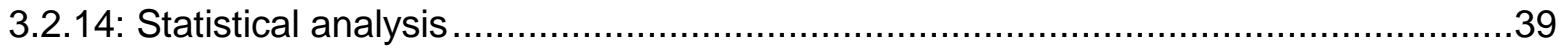

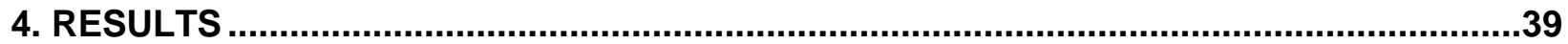

4.1: Acute E. coli infection and EAE.......................................................................39

4.1.1: Intraperitoneal infection with $E$. coli induces systemic inflammation .......................39

4.1.2: The influence of $E$. coli infection on clinical course of MOG-EAE .......................40

4.1.3: Infection with E. coli and neuropathological damage of the optic nerve .................41

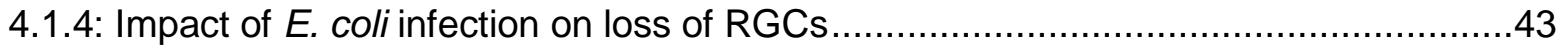

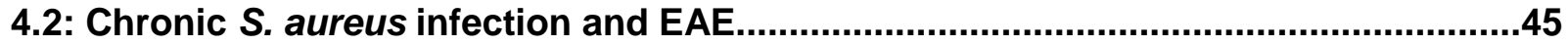

4.2.1: Local infection with $S$. aureus leads to persistent systemic inflammation................45

4.2.2: Infection with $S$. aureus enhanced lymphocyte counts in peripheral blood .............47

4.2.3: Chronic S. aureus infection and disease activity in MOG-EAE ...........................49

4.2.4: Effect of $S$. aureus infection on neurodegeneration ....................................49

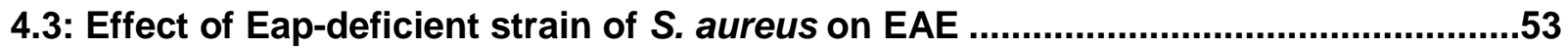

4.3.1 Infection with S. aureus strain deficient for Eap and systemic inflammation .............53

4.3.2: Infection with $S$. aureus and cytokines release in the systemic circulation...............54 
4.3.3: S. aureus infection and its impact on clinical manifestation of the disease

4.3.4: Neuropathological damage and RGCs survival after Infection with Eap-deficient strain of $S$. aureus.

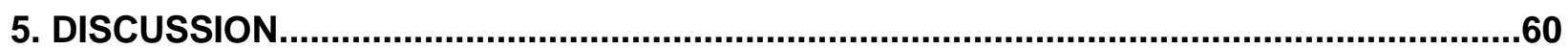

5.1: Infection and MS............................................................................................60

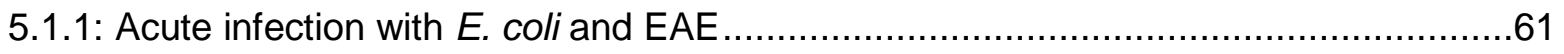

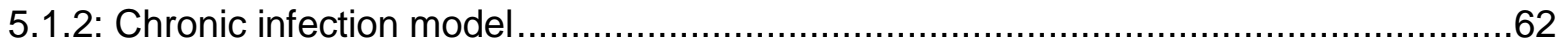

5.1.3: Chronic infection with S. aureus, induces systemic inflammation .......................63

5.1.4: Chronic infection with S. aureus and cytokine release ...................................65

5.1.5: The role of adhesion molecules in transendothelial migration...........................66

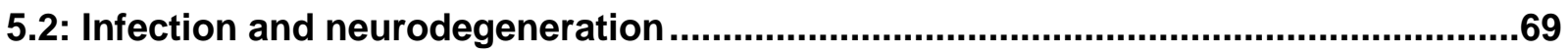

5.3: A clear distinction between the animal model of MS is important.........................71

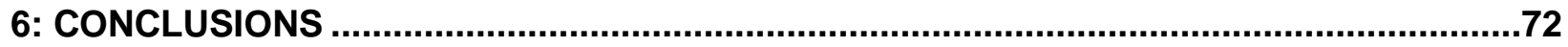

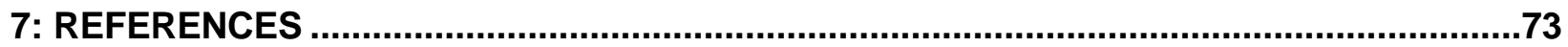

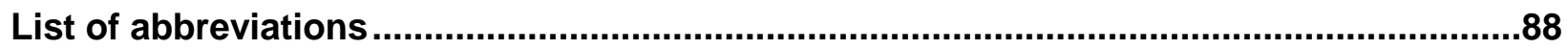

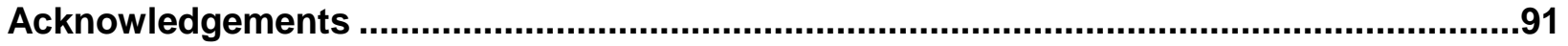

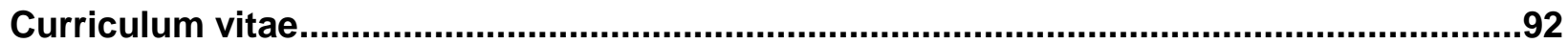




\section{ABSTRACT}

The course of Multiple Sclerosis (MS), a chronic autoimmune disease, can be influenced by systemic infections. The common notion is that bacterial infections worsen disease symptoms and lead to enhanced neurodegeneration. However, the underlying biological mechanisms by which bacterial infections may lead to neurodegeneration in MS are complex and poorly understood. Here, we assessed the disease-modulating effect of an acute Escherichia coli and a chronic Staphylococcus aureus infection on the clinical course and the extent of neurodegeneration in experimental autoimmune encephalomyelitis (EAE), an animal model of MS.

In this study, we did not observe any disease modulating effect of acute $E$. coli infection on EAE when infection was induced in both the preclinical and in the clinical phase of the disease. However, we observed that local application of $S$. aureus in the preclinical phase of the disease induced a chronic systemic inflammatory response with increased in T- and B-cell counts as well as systemic production of pro-inflammatory cytokines. Unexpectedly, the infection with $S$. aureus completely prevented the development of clinical EAE. Moreover, it markedly decreased the extent of inflammatory infiltrates and demyelination of the optic nerve and increased the number of surviving retinal neurons. Using a $S$. aureus strain deficient for the extracellular adherence protein (Eap) we identified this protein to be at least partly responsible for the inhibitory effect of $S$. aureus infection on autoimmune inflammation of the central nervous system. Thus our results show for the first time a beneficial effect of a chronic bacterial infection on neurodegeneration in EAE and open the avenue for the design of new neuroprotective therapy in MS. 


\section{INTRODUCTION}

\section{1: Multiple sclerosis: General background}

Multiple sclerosis (MS) is an autoimmune, inflammatory, demyelinating disease of the central nervous system (CNS), which often manifests with optic neuritis (Hickman et al., 2002). MS was first described in detail by Dr. Jean-Martin Charcot in 1866 and called it as "sclerose en plaques" (Compston, A. 1998). The main target of damage in MS is the myelin sheaths which are wrapped around the neurons and make efficient conduction of nerve impulse. During the disease course, there is a destruction of the myelin sheath which may lead to the impairment of impulse along the axons which further cause neurological abnormalities and typically affect motor, sensory and visual functions (Compston and Coles, 2008).

It is estimated that about 2.3 million people are affected by MS and the number of patients are increasing over the time (MS Atlas 2013). MS is present worldwide, but the prevalence of MS varies in different parts of the world. Earlier studies have indicated that the incidence of the disease increased with the distance from the equator (Kurtzke, 2005; Miller et al., 1990; Simpson et al., 2011). The highest prevalence of MS is seen in people from North America and Europe (>100/100 000) and it is rare amongst Asian and African (2/100 000) (MS Atlas 2013). However, the prevalence of MS also varies considerably within regions (Compston and Coles, 2008).

MS is the most common neurological disease of young adults and females are twice more affected than males (Alonso and Hernan, 2008; Rezaali et al., 2013). Although with lower incidence rate of MS in male, they show slower recovery and show more aggressive nature of the disease course than females (Koutsis et al., 2010; Leibowitz and Alter, 1970; Runmarker and Andersen, 1993; Weinshenker et al., 1991). Due to early onset, the disease has a huge impact on the socioeconomic status of an individual. The majority of MS patients are diagnosed between the age of 20 and 50 years (National MS society). The first clinical symptoms can include fatigue, headache, limb weakness, vision abnormalities, impairment of bladder, depression and problems 
with balance and coordination (Schneider, 2005). Clinical onset of disease is characterized by the neurological relapses followed by remission and this type of disease is referred as relapsing-remitting type of MS (RRMS) and found amongst 80 $85 \%$ of MS patients (Gold et al., 2010; Rovaris et al., 2006). RRMS patients are clinically stable between the relapse for several years. However, there is an increasing evidence to suggest that the axonal and neuronal loss occurs in the early phase of MS (Kornek and Lassmann, 1999; Trapp et al., 1998; Trapp et al., 1999). The majority of RRMS patients further develop secondary progressive MS, (SPMS) where patients show axonal loss and neurological deficit without relapses (Fitzner and Simons, 2010; Rovaris et al., 2006). This could lead permanent cause of neurological disability of MS patients. Approximately $10-15 \%$ of patients has no remitting phase and show progressive worsening of the disease from the start and called as primary progressive MS, (PPMS) (Andersson et al., 1999; Miller and Leary, 2007). Recent advances in magnetic resonance imaging techniques improved all over the world which added the value in the diagnosis of MS patient by characterizing the lesions size and locations directly into the white matter of the brain in very early stage of disease (Patsopoulos et al., 2011). Interferon-beta (IFN- $\beta$ ), glatiramer acetate (GA), and natlizumab, are some common medication used for the treatment of MS. Since 2010 fingolimod (FTY 720) was approved for the treatment of MS as a first oral drug. These drugs slow down the progression of disease, but they could not prevent the neuronal cell loss and in the consequences of this ongoing progression of neuronal loss patients develop the long term disability. Therefore there is a need to design of new neuro-protective therapy in MS.

\section{2: Pathogenesis and pathology}

MS predominantly assumed to be a T-cell mediated autoimmune disease (Wekerle, 2008) involving both $\mathrm{CD}^{+} \mathrm{T}$ helper-cell and $\mathrm{CD} 8^{+} \mathrm{T}$ cytotoxic-cell (McFarland and Martin, 2007). In the recent years the role of B-cells is also emphasized in MS pathogenesis (Hirotani et al., 2010). Although the sequential event taking place in the pathogenesis of MS is hardly known, but activation of auto reactive T-cells is supposed to be the initial step in the disease pathogenesis (Markovic-Plese et al., 2004). 
However, factors which trigger this phenomenon are not exactly known. From several clinical studies myelin reactive T-cells has been shown to be present in every individual. But these myelin-reactive T-cells, reactive to myelin basic protein (MBP), were found to be significantly higher in MS patients compared to healthy controls (Zhang et al., 1994). Thus it is presumed that some environmental factors, especially the microbial antigens, which probably supposed to cross-activate T-cells specific for myelin epitopes through a mechanism called molecular mimicry in susceptible individuals (Wucherpfennig and Strominger, 1995). During the activation cascade T-cell receptor present on T-cell recognizes and binds to the myelin specific antigen present on major histocompatibility complex (MHC) of an antigen-presenting cell (APC) which delivers co-stimulatory signal leading to activation of myelin specific T-cells (Fig 1.1). These activated T-cells cross the blood-brain barrier (BBB), a physical barrier formed by the endothelial cells that form the walls of the capillaries, and enter into the CNS.

The BBB maintains highly controlled microenvironment of the CNS by regulating several functions across the CNS like passive diffusion of nutrition, maintenance ionic balance, prevention of macromolecules and neurotoxins to enter into the CNS. During several neurological disorders and in traumatic brain injury the BBB is breached, which allows the inflammatory cells to enter into the CNS and impair the neuronal function (Rosenberg, 2012). The interactions between the adhesion- and signaling-molecules present on leukocyte and endothelial cells lining the BBB leads to the recruitment of pro-inflammatory cells to the CNS (Rossi et al., 2011). That includes the involvement of cytokines, chemokines and adhesion molecules expressed by both the activated endothelium and the immune cells, which especially involve the very late antigen-4 Vascular cell adhesion molecule-1 (VLA-4 - VCAM-1) and the lymphocyte functionassociated antigen-1-intercellular adhesion molecule-1 (LFA-1 - ICAM-1) adhesion systems (Xie et al., 2006). Monoclonal antibody like natalizumab is in therapeutic use for MS, which targets interaction between VLA-4 - VCAM-1, and subsequently block the migration of activated T-cells across the BBB and reduces the severity of disease. Although of a majority of T-cells are crossing the BBB primarily through VLA-4 - VCAM1 adhesion pathway, but the LFA-1 - ICAM-1 also plays an important role in downstream to this pathway. 


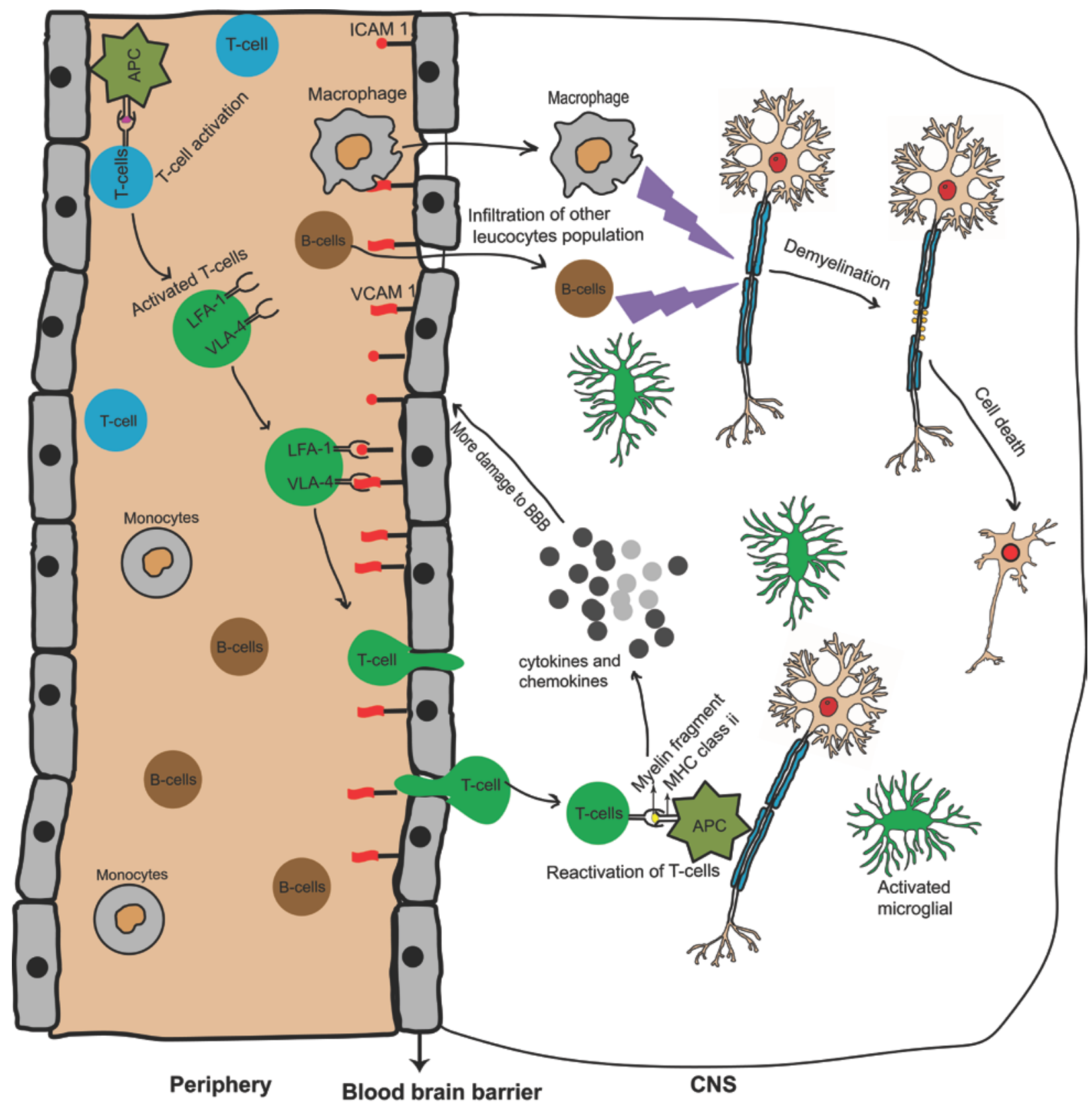

Fig 1.1: Immunopathogenesis of MS: Initial activation of T-cells takes place in the periphery by antigen presenting cells (APCs) followed by transendothelial migration of activated T-cells across the blood-brain barrier by use of different adhesion system. After crossing the BBB these cells undergo reactivation within the CNS and induce the release of several pro-inflammatory cytokines, which further cause damage to the BBB. The damaged BBB allows the other blood cells to enter into the CNS. That together with other brain cells contribute to the damage of the myelin sheath and ultimately to the death of the neuronal cell bodies. 
It has been also suggested that, the integrity of the BBB could be protected by blocking the infiltration of inflammatory cell recruitment secondary to the activated T-cells (Xie et al., 2006). Several studies indicated the role of ICAM in pathogenesis of MS (Bullard et al., 2007; Steiner et al., 2010). Eventually there is no current therapy targeting the LFA1 - ICAM-1 adhesion pathway. However, some microbial products like extracellular adhesion protein (Eap), secreted from staphylococcus aureus (S. aureus) have been shown to have potential which specifically targeted LFA-1 - ICAM-1 but not VLA-4 VCAM-1 mediated T-cell recruitment at the BBB in an animal model of MS (Xie et al., 2006).

After crossing the BBB, T-cells get reactivated by antigen presented on resident brain antigen presenting cells (APCs), which triggers the release of pro-inflammatory cytokines (Th1 cytokine profile) like Interleukin (IL)-1, IL-2, IL-6, IL-12, IFN-gamma and TNF- $\alpha$ that further disrupt the BBB and resulting in a massive infiltration of other inflammatory cells from the peripheral blood circulation. The inflammatory cells further destroy the myelin sheath of the neurons and subsequently cause neurodegeneration and plaque formation in the CNS (Fig 1.1).

Since multiple sclerosis is clinically, genetically and pathologically heterogeneous disease, one can assume that more than one pathogenic mechanism leading to the disease progression. It has been shown that axonal injury and neuronal cell loss occurs in the early phase of MS lesion formation and is most pronounced in active demyelinating phase of the disease (Kuhlmann et al., 2002). This slow and continuous axonal destruction may account more to the global axonal loss in MS.

A pathological hallmark of MS is multiple focal areas of myelin loss called plaques, especially in the white matter of the CNS (Ge, Y. 2006). Inflammation, demyelination, oligodendrocyte injury, astrogliosis, neurodegeneration and axonal loss are the main factors associated with the plaque formation. MS plaques, may develop anywhere in the CNS, but most frequently affected areas are the optic nerves, spinal cord, brainstem, peri-ventricular white matter regions and cerebellum (Popescu and Lucchinetti, 2012). Plaques can be in acute or in chronic form that depends on the degree of inflammation present within the plaques. Acute plaques are more common in 
patient with RRMS, which shows robust inflammatory infiltration with lymphocytes (predominantly T-cell) macrophages and distributed throughout the lesions and shows parenchymal edema (Frohman et al., 2006) that can be detected by gadolinium enhancement on MRI. Moreover, these plaques also contain a considerable number of oligodendrocyte precursor cells, which probably help the CNS in the restoration of myelin (Wolswijk, 1998).

Once the inflammation in the acute plaque resolves in the brain or spinal cord it forms scars and turned into a chronic form of plaque and accumulates throughout the disease course. Chronic plaques are more common in patients with progressive MS (Kutzelnigg and Lassmann, 2005). These plaques show complete demyelination, substantial loss of oligodendrocytes and axons, astrogliosis and less infiltration of lymphocytes and macrophages (Popescu and Lucchinetti, 2012).

\section{3: Genetics and role of infection}

The exact cause of this highly complex disease is still unknown. It has been suggested that both genetic and environmental factors are the two major determinants which contribute to the disease susceptibility. However, the precise impact of these factors is not very clear so far and that can be either harmful or protective.

Demographic studies and data from the epidemiology suggest a strong influence of genetic factors in pathogenesis of MS (Barcellos et al., 2006; Chataway et al., 2001; Dyment et al., 1997; Sawcer et al., 2011; Weinshenker, 1994). Human leukocyte antigen (HLA) locus, present on the short arm of chromosome 6, in major histocompatibility complex (MHC) region was discovered as a first potential genetic risk factor associated with the pathogenesis of MS in 1970s (Munoz-Culla et al., 2013). These genes encode the cell surface glycoproteins, which are the key components of the immune system. Since 1970s a great deal of effort has been made to understand the functional and mechanistic role of the genes present in this locus (McElroy and Oksenberg, 2011). From a collaborative effort of Wellcome Trust Case Control Consortium 2 and International Multiple Sclerosis Genetics Consortium, containing large data set, it has been shown that HLA DRB1*15: 01 loci have the strongest association 
with multiple sclerosis and also identified 29 novel susceptibility loci (Sawcer et al., 2011). Even though with the strong genetic evidences it is also noticed that in $75 \%$ of the population only one sibling getting affected with MS which signifies the role of additional factors which play an important role in the pathogenesis of MS (Ebers et al., 1986; Sadovnick, 1993; Sadovnick et al., 2009).

The idea of MS pathogenesis and the role of infection came in late 1800s by Pierre Marie, and since then a number of different pathogens have been associated and then discarded due to lack of evidences. Many clinical studies have shown that common bacterial and viral infections that cause respiratory infections and urinary tract infections may alter the immune status of an individual. However, in regards to MS and its animal model, it is observed previously that infections with these bacteria and viruses significantly influence the course and severity of MS and experimental autoimmune encephalomyelitis (EAE) especially immediately after viral and bacterial infections (Tauber et al., 2007).

Epstein Barr virus (EBV) infection is most promising candidates, which are gaining support for being involved in MS pathogenesis. The link between EBV and MS was come in picture from a study of Warner and Carp in 1981, who suggested that the young adults are at highest risk of acquiring MS where the chances of infectious mononucleosis are at most (Warner and Carp, 1981). EBV infection occurs in over 90\% of the general population, but in an epidemiological study conducted in the past 30 years revealed that MS patients are almost universally, seropositive for EBV infection (Pender, 2011). Antibodies against EBV antigens particularly, Epstein-Barr nuclear antigen-1 was detected in the cerebrospinal fluid (CSF) of the MS patients in significantly higher amount compared to the control (13\% of controls against $85 \%$ of MS patients, p<0.001) (Bray et al., 1992; Cepok et al., 2005; Jaquiery et al., 2010; Rand et al., 2000). There are several other viral studies which are linked to the etiology of MS like human herpesvirus-6 (Challoner et al., 1995; Kim et al., 2000; Opsahl and Kennedy, 2005; Panitch, 1994), adenoviruses (Andersen et al., 1993), picornavirus (Kriesel et al., 2004), varicella-zoster virus (Mancuso et al., 2007; Ordonez et al., 2004) and torque teno virus (Sospedra et al., 2005). 
Even though viral particle can cause the infection of the brain directly, but in most of the studies researchers could not found presence of viral particle in the brain. Moreover, several patients have been documented until now and they were sero-negative for these viruses. Hence these results suggest that these viruses are the strong risk factors for the pathogenesis of MS rather than a direct cause for acquiring this disease.

Earlier studies with bacterial infection and MS were not given importance due to development of thrust (Sibley and Foley, 1965). However, in recent time, studies with the animal model and MS patient shows that bacterial infection may lead to exacerbations in MS (Buljevac et al., 2002; Herrmann et al., 2006). The possible explanation for these exacerbations is that, an infection may increase the inflammatory activity in the peripheral vascular system which may lead to more sustained neurological damage over a period of time. Thus these bacterial components appear to play an important role in MS pathogenesis and were found also in the CNS of MS patients (Schrijver et al., 2001). In a study by Sriram and colleagues in 1999, it was observed that $97 \%$ of CFS samples from MS patients were found positive for Chlamydia pneumoniae (C. pneumoniae) major outer membrane protein gene against $18 \%$ of control. Moreover, from the culture of the CSF they showed the presence of $C$. pneumoniae in $64 \%$ of the MS patients against $11 \%$ from control patients. In contrast, some bacterial component seems to prevent the EAE (Ben-Nun et al., 1995; Ben-Nun and Yossefi, 1992).

In the last few decades with the increase in the sanitary condition in western countries showed a tremendous increase in the incidence of many immune disorders including MS (Bach, 2005). Epidemiological evidence indicates a strong correlation between improved sanitation and an increase in the number of patients with such immune disorders (Fig 1.2) (Bach, 2002; Christen and von Herrath, 2005). Hence this increase in the immune disorder against high sanitation level gives rise to new hypothesis known a hygiene hypothesis, first proposed by Strachan for allergic disorders (Strachan, 1989) and now correlates well with several other autoimmune disorders. According to the hygiene hypothesis, the children grows in the highly hygienic environments have negative effect on their immune development which may lead to an 
in the incidence of autoimmune disorders like MS (Fleming and Cook, 2006; Leibowitz et al., 1966). This finding also supported by migration studies which indicate that moving from a high- to a low-risk area in childhood reduces the risk of MS, while moving during late adulthood has no impact on susceptibility of MS. Migration in the opposite direction, does not seems to increase the MS risk substantially until the next generation (Ascherio and Munger, 2007; Gale and Martyn, 1995; McLeod et al., 2012; Orton et al., 2010).
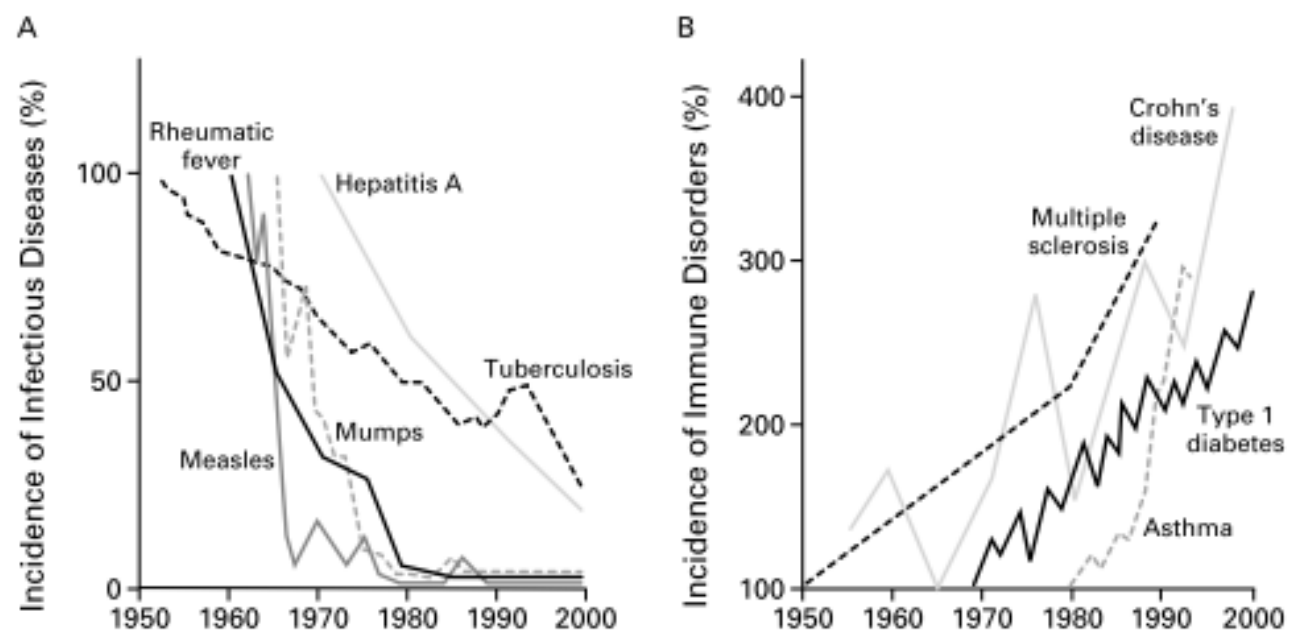

Fig 1.2: Infectious disease and autoimmune disorder: Epidemiological data indicate an inverse relation between the Incidence Infectious Diseases (A) and the Incidence of Immune Disorders (B) from 1950 to 2000 (Bach, 2002).

These data suggest that an environmental factor, like infection in childhood is important, to determine MS risk. The possible mechanism behind this protection might be that, infection may shift the immunological balance toward a more immunesuppressed state or infection may cause hyper activation of auto reactive cells which possibly lead to clonal deletion and ultimately reduces the number of aggressive cells. Recent epidemiological investigations with Trichuris trichiura, a common human pathogen, indicate that MS prevalence appears to fall sharply once a critical threshold of Trichuris trichiura is exceeded (Fleming and Cook, 2006). These studies in humans are validated by investigating the impact of some other parasites in animal models of MS. In one such study animals were pre-infected with the ova of Schistosoma mansoni which results in either attenuation or delayed onset of EAE (La Flamme et al., 2003). Studies also indicate that the infection with the ova of Schistosoma mansoni 
significantly down regulates the induction of some pro-inflammatory cytokine such as IL12, TNF-alpha (TNF- $\alpha$ ), IFN-gamma and express more anti-inflammatory cytokine like IL-10 and TGF-beta. Moreover, these studies also indicate the role of regulatory T- and B-cells in the disease onset. Although lots of research are ongoing on the parasitic infection and viral infection to their relation to the autoimmune diseases like MS, but eventually the role of bacterial infection and their relation to the MS still need to be explored.

\section{4: Diagnosis and therapy}

A careful medical history and a thorough neurological examination form the basis for treatment of MS patients. There are some defined criteria made in the diagnosis of MS which is changed and modified over time. First diagnostic criteria used for MS was proposed by Schumacher in 1956 (Ross et al., 1965) which were updated by Poser criteria in early 1980s. Poser criteria first emphasized the use of MRI for diagnosis of MS patient. This criteria also included the analysis of cerebro-spinal fluid analysis (CSF) examination (Poser et al., 1983). The poser criteria was used for several years and updated by "McDonald Criteria" in 2001 (McDonald et al., 2001) and revised time to time. The latest revision of McDonald criteria was made in 2010 by (Polman et al., 2011). According to the latest revision of this criteria if a patient has had more than two attacks, or having more than two lesions, or one lesion but with sufficient evidence of a prior attack, can be diagnosed, if the patient does not fulfill these criteria the further attack is needed for the secure diagnosis (Polman et al., 2011). The McDonald criteria make use of advancement in MRI techniques which allows the diagnosis to be accurate at earlier stage of disease, and therefore offer the potential for earlier treatment. The expanded disability status scale (EDSS) is used worldwide in clinics to evaluate the disease progression in MS, where higher EDSS scores indicate more disability. Since 2001 McDonald criteria made the foundation to diagnose MS in most of the countries.

Over the last 20 year, several therapeutic agents have been studied and tested for the MS treatment. However, immune suppressive therapy becomes the first choice for the attenuation the disease progression probably due to inflammatory nature of this 
disease. The first line treatment consists of interferon- $\beta$, which is either IFN $\beta-1 \mathrm{a}$ or IFN $\beta-1 b$ and glatiramer acetate (GA) for the RRMS.

IFN- $\beta$ treatment is administered sub-cutaneousaly showed a reduction in the relapse rate by $30 \%$ and reduce lesion load (Jacobs et al., 1996; Jacobs et al., 1995). Although the exact mechanism of action is not clearly understood until now, but it was suggested from several studies that INF- $\beta$ therapy reduces the expression of MHC class II on APCs, reduces proliferation of T-lymphocytes and the production of anti-inflammatory cytokines (Dhib-Jalbut and Marks, 2010; Genc et al., 1997; Jiang et al., 1995; Teleshova et al., 2000), increases cytotoxic T-lymphocyte and Fas surface molecule on $\mathrm{CD}^{+}$cells and promote apoptosis of aggressive cells (Hallal-Longo et al., 2007). Other disease modifying drugs heavily in use is glatiramer acetate (GA), a four-amino acid synthetic copolymer administered sub-cutaneoualy and have been shown to reduce the relapse rate by $29 \%$ (Johnson et al., 1995). The precise mechanism of action of GA is not clearly known, but several studies indicate that GA leads to an inhibition of the antigen- specific T- lymphocyte activation by binds directly to the portion of the APC. Due to lower efficacy of these drugs, escalation therapy for the treatment MS was introduced which primarily includes natalizumab (humanized monoclonal antibody) and fingolimod (sphingosine-1 phosphate receptor agonist). Natalizumab targets a component of the VLA-4, a4-integrin molecule which is present on lymphocytes thus prevent the interaction with VCAM-1 found on endothelial surfaces and subsequently blocks the infiltration of these lymphocytes across the BBB which results in reduced inflammation of the CNS. Due to a very high efficacy (Miller et al., 2007; Polman et al., 2006) and severe side effect of natalizumab (Bloomgren et al., 2012; Clifford et al., 2010), several other monoclonal antibody directed therapy (Alemtuzumab, Ocrelizumab) is under trial for MS treatment. A sphingosine-1 phosphate receptor agonist, Fingolimod (FTY720), which blocks leukocyte trafficking in lymph nodes, is the first oral disease-modifying treatment available for relapsing MS and reduces the relapse rate by $54 \%$ and shows a significant decline in MRI lesion (Kappos et al., 2010). Mitoxantrone an anthracenedione cytotoxic agent can cross the disrupted BBB and inhibits DNA replication and reduces lymphocyte proliferation. Although mitoxantrone is effective in MS treatment, it remains second line for RRMS due to serious adverse 
effects. The role of B-cell has been explored in the MS and several therapies are on trial now a day's targeting B-cell populations and such hypothesis leads to the development of Rituximab and Ocrelizumab (van Oers et al., 2010; Zhou et al., 2008).

Although in the last few years there is a huge intensification of MS therapy and have shown very high efficacy, especially in the RR form of MS but none of the marketed anti-inflammatory therapies are approved for PPMS or SPMS (Cree, 2014). Moreover, there is no treatment option available, which makes the neurological impairment reversed. More studies are needed to develop therapies which could control neurodegeneration, which is the main cause of permanent neurological disability.

\section{5: Experimental autoimmune encephalomyelitis}

Since the CNS is hardly accessible in humans for molecular and biochemical studies, therefore to assess the impact of a treatment or infection on MS various animal models have been used so far. These experimental animal models have provided important information in our understanding of various human autoimmune diseases which allow us to investigate the influence environmental and genetic factors associated with the disease progression. These animal models also provide a different experimental therapeutic approach to test the impact of a particular drug before going to trial in human.

Although none of the animal models replicates all the immunological and histopathological aspects of MS. However, experimental autoimmune encephalomyelitis (EAE) is one of the most commonly used models of MS (Wekerle et al., 1994) and shows several immunological and histopathological aspects of MS. EAE is an inflammatory autoimmune disease of CNS clinically manifested by the development of a tail and limb paralysis which reflect the histopathological resemblance to MS (Goverman et al., 1993; Swanborg, 1995). Depending on the strain of the animals an EAE can be induced in animals models by immunization with various myelin proteins like proteolipid protein (PLP), myelin oligodendrocyte glycoprotein and myelin basic protein (MBP) along with the adjuvants (Gold et al., 2006). The most commonly used adjuvant is Freund's adjuvant which contains mineral oil together with heat killed Mycobacterium 
tuberculosis. To induce EAE, pertussis toxin is also used in combination with myelin proteins several animal model. The EAE can also be induced by adoptive transfer of myelin specific activated T-cells (Baxter, 2007; Zamvil and Steinman, 1990).

MOG is a myelin surface antigen, highly conserved between species (Gardinier et al., 1992; Kroepfl et al., 1996) and present on the outer lamella of the myelin sheath and is recognized by activated T-lymphocytes and demyelinating antibodies. The precise biological function of MOG is not known, although it has been speculated that it has an adhesive function between myelin sheets and provides microtubule stability in oligodendrocytes. Immunization with recombinant myelin oligodendrocyte glycoprotein leads to the activation of MOG specific T-cells, which further reaches to the secondary lymph nodes where these cells get proliferated and migrated to the CNS by crossing the BBB (Cannella et al., 1990). Migration of these activated T-cells cause autoimmune inflammation of the CNS and release of pro-inflammatory cytokine which induces more damage to the BBB. The breakage of BBB allows other peripheral blood cells like Bcells, monocytes and macrophages to enter into the CNS, which primarily attack the myelin sheath of neuron (Traugott et al., 1986).

In this thesis dissertation, we have used MOG (extracellular domain of rat MOG amino acid sequence 1-125) immunized female Brown Norway (BN) rats as an animal model. After an active immunization with MOG this animal model shows severe optic neuritis with acute axonal degeneration of the optic nerve (ON) and consecutive apoptosis of retinal ganglion cells (RGCs) in more than $90 \%$ of the rat regardless of a clinical manifestation of the disease (Hobom et al., 2004; Maier et al., 2006; Meyer et al., 2001). Therefore, this EAE model provides a unique tool to investigate the morphological changes in neuronal cell bodies and in axons.

\section{6: Infection models}

Epidemiological studies suggest that MS is greatly influenced by environmental factors like infection. The role of viruses in MS etiology is highly convincing. Moreover, it is also shown that bacterial components like enterotoxins, LPS and peptidoglycans are able to influence the course of EAE (Moreno et al., 2011; Schiffenbauer et al., 1993; Soos et 
al., 1995). But the role of common bacterial infections has hardly been explored which reflect the true clinical situation. Although in most studies, the effects of acute infection were examined, but in the best of my knowledge the impact of chronic bacterial infection was never been evaluated which more closely mimic the clinical situation in humans. Therefore in this thesis dissertation we evaluated the impact of both acute and chronic infections in MOG immunized female BN rat.

\subsection{1: Acute infection model}

Despite of strong stimulatory effect of $S$. aureus enterotoxins on human (Langford et al., 1978; Peavy et al., 1970) and mouse (Janeway et al., 1989; White et al., 1989) Tlymphocyte subsets, it was unable to stimulate proliferative responses of rat Tlymphocytes in a dose dependent manner (Ben-Nun and Yossefi, 1992). The studies with these bacterial components induce different responses towards different animal models. Such studies cannot be correlated with the normal infectious condition in MS or EAE.

Du and coworkers (2002) showed in mice that intraperitoneal infection with $C$. pneumoniae can aggravate symptoms of EAE. This effect is likely to occur, since infection of the CNS increases the auto-reactive lymphocyte pool, hence influenced the development of autoimmune disease. However, in the same study infection with Chlamydia trachomatis did not influence the clinical course of disease. This study indicates that a CNS infection would thus essential for a worsening of EAE and it is also likely possible that the impact of infection on EAE varies with infection with different microorganisms. Hermann and colleagues in 2006 did an experiment where they infected the animals with Streptococcus pneumoniae at different time point after immunization in EAE. They observed that infection was able to exacerbate EAE only in case when the infection was made on day 7 post immunization (early asymptomatic phase). However, they did not observe any influence of infection on EAE when infection was made on 2 days prior to immunization or during the late phase of the disease (day 21 pi). This study not only indicates the influences of infection on EAE rather the importance of infection induced at different time points during the course of the disease 
are equally important. Furthermore, they also observed that bacterial infection enhances the release of pro-inflammatory cytokines in peripheral blood circulation. As indicated in previous studies that TNF- $\alpha$ (Murphy et al., 2002; Ruddle et al., 1990) alpha and IL-6 (Samoilova et al., 1998) plays an important role in worsening of EAE. The increase in the level of TNF- $\alpha$ and IL- 6 was also observed in the study of Herman et al.

Research evidences over a period of time also suggested that infectious agents may play a protective role in autoimmune diseases like MS. The inhibitory effect of bacterial infection in EAE and MS has also been observed (Ben-Nun et al., 1995; BenNun and Yossefi, 1992). Ben-Nun and collogues has shown that the SJL/J mice preexposed to Mycobacterium tuberculosis (Mt) and some other bacterium, developed long-lasting resistance to EAE and also identified a 12-kDa protein fraction, belongs to heat shock protein family, to be responsible for this protective effect. They also speculated that the protection in these mice from EAE arises due to the result of similar T-cell epitopes between mycobacterial heat shock protein and different myelin proteins. Moreover, some role of parasitic infection also has proven protective in EAE, which generally shift the immunological balance towards $\mathrm{Th}_{2}$-cell type. From the above research evidences it could be speculated that infections increases the natural propensity of these animal models, hence they developed resistant towards an autoimmune disease.

To induce a mild infection and to increase the survival of animals after infection, they are generally treated with antibiotics. Antibiotic treatment seems to affect the EAE in both ways, some antibiotics seems to be protective in EAE while some are not (Wang et al., 2009). The precise mechanism of how antibiotic treatment influences EAE is not clear so far. Through a short term treatment in any case seems to have no direct neuroprotective effect in the EAE (Herrmann et al., 2006). However, antibiotic treatment like ceftriaxone may cause rapid degradation of bacterial components which may probably do not reflect the normal infectious condition. Therefore the impact of infection in animal models should be evaluated without intervention of any antibiotic treatment. Hence for that reasons, chronic infection model without any antibiotic treatment would be more appropriate to evaluated impact of infection in EAE. 


\subsection{2: Chronic infection model}

Infections in prostheses lead to serious complications. This is due to the formation of a bacterial biofilm, antibiotic tolerance and an altered activity of phagocytic cells associated with the foreign body. The main causative agent for this is $S$. aureus. Therefore, by taking advantage of this microorganism Werner Zimmerl and co-workers in 1982 established an experimental model chronic foreign body infection in guinea pig to study pathogenesis of foreign body Infection (Zimmerli et al., 1982). Later on Lucet et al established this model in Wistar rat. A chronic infection is achieved by implantation of a foreign body, in which the bacteria can adhere (Lucet et al., 1990; Murillo et al., 2006). In the study of Lucet et al tissue cages were implanted under the skin and infected with S. aureus to test the effect of different antibiotics. The inoculum dosage of $10^{5}-10^{6}$ colony forming unit (CFU) was used to establish this infection model. However, this inoculums dosage may vary with the depending on the strain of the rat.

Shapira and colleagues (2002) implanted tissue cages made of titanium wire in mice and immunized them 2 weeks later with mouse spinal cord homogenate (MSCH). Five days after immunization, animals were infected with heat killed Porphyromonas gingivalis, which resulted in a more severe EAE (Shapira et al., 2002). They concluded that this effect is due to more peripheral inflammation. However, infection with heat killed bacterium does not represent the true infectious condition and does this infection leads to the chronic systemic inflammation was also not clear. Hence in best of my knowledge, there is no such study with the true chronic bacterial infection on an animal model of MS has been performed until now. 


\section{AIM OF THIS WORK}

The course of Multiple Sclerosis, a chronic autoimmune disease, can be influenced by systemic infections. The common notion is that, bacterial infections worsen disease symptoms and to lead to enhanced neurodegeneration. However, the underlying biological mechanisms by which bacterial infections influence the disease course and neurodegeneration in MS are complex and poorly understood. Most of the previous experimental studies on animal model used the bacterial component to induce the systemic inflammation to mimic the situation of viable bacterial infection. Further, the Inflammation induced by the bacterial components or by a bacterial infection induces similar effects on the course of the disease has not yet proven. Furthermore, the kind of infection (acute and chronic) even more the type of bacteria used may also a have varying impact on the course of disease and neurodegeneration.

So our objective in this study was

1. To investigate the influence of acute Escherichia coli (E. coli) infection on the clinical course and the extent of neurodegeneration in experimental autoimmune encephalomyelitis, when infection was induced during preclinical and clinical phase of disease

2. Establish a chronic infection model in female $\mathrm{BN}$ rats

3. To investigate the influence of chronic Staphylococcus aureus (S. aureus) on the clinical course and the extent of neurodegeneration in experimental autoimmune encephalomyelitis, when infection was induced in the preclinical phase of disease 


\section{MATERIALS AND METHODS}

3.1: Materials: - Materials used in this thesis are listed in the following tables. Unless otherwise stated, materials were purchased from companies located in Germany.

\subsection{1: Instruments}

Cell counting chamber

Neubauer cell chamber

Superior-Marienfeld

\section{Centrifuges}

Allegra $\times 22 \mathrm{R}$

Allegra

$5415 \mathrm{C}$

Eppendorf

$5425 \mathrm{R}$

Eppendorf

\section{Cryotome}

CM 30505

Leica

\section{Luminex}

Bio-Plex 200

Bio-Rad

Fridge and freezers

GNP $3656\left(4^{\circ} \mathrm{C}\right)$

Liebherr

KDR40420GB/01 $\left(4^{\circ} \mathrm{C}\right)$

Bosch

GSS $3626\left(-20^{\circ} \mathrm{C}\right)$

Liebherr

KSD 3142 Premium $\left(-20^{\circ} \mathrm{C}\right)$

Liebherr

GNP $3056\left(-80^{\circ} \mathrm{C}\right)$

Liebherr

\section{Flat cooler}

TUC2

Pathhisto

\section{Flow cytometer}


High thermal flattening table

HI 1220

Leica

Hood

Laminar air hood

Thermo Scientific

Magnetic stirrer

$\mathrm{RH}$ basic

IKA

IKAmag reo

IKA

\section{Microscope}

Axiopaln 2

Carl Zeiss

axiovert 200

Carl Zeiss

Axiostar plus

Carl Zeiss

Stemi $2000-C$

Carl Zeiss

Examiner Z1

Carl Zeiss

\section{Microtome}

SM2000R

Leica

\section{Pipette}

$2.5 \mu \mathrm{l}, 10 \mu \mathrm{l}, 100 \mu \mathrm{l}, 200 \mu \mathrm{l}, 1 \mathrm{ml}, 5 \mathrm{ml}$

Eppendorf

$2.5 \mu \mathrm{l}, 10 \mu \mathrm{l}, 100 \mu \mathrm{l}, 200 \mu \mathrm{l}, 1 \mathrm{ml}$

Gilson

$50 \mu \mathrm{l}, 200 \mu \mathrm{l}$ (Multichannel)

Eppendorf

pH Meter

PB-20

Sartorius

\section{Vortex}

MS 3 basic

IKA

Vortex-Genie 2

IKA

\section{Weighing balance}

BL2105

Sartorius

BL 3100

Sartorius 


\subsection{2: Consumables}

\begin{tabular}{|c|c|}
\hline Name & Company \\
\hline Aluminium foil & Carl Roth \\
\hline Blood agar plates & Department of Microbiology Göttingen \\
\hline Centrifuge tubes (0.5-, 1.5-, 2-ml) & Eppendorf \\
\hline Centrifuge tubes $(15-50 \mathrm{ml})$ & BD Falcon \\
\hline Cover slip $(24 * 48,24 * 60)$ & Menzel-glaser \\
\hline Cuvette & Carl Roth \\
\hline Filter tip (10-, 200-, 1000-,1250- $\mu$ l) & Greiner Bio One, Sarstedt \\
\hline Gloves & Meditrade \\
\hline Microscope slides (Superfrost plus) & Thermo Scientific \\
\hline Microtome blade & Feather \\
\hline Needles (28G, 23G, 27G) & BD Eclipse \\
\hline Nitrile gloves & Meditrade \\
\hline Nunc 96 well plates & Apogent \\
\hline Para film & Pechiney Plastic Packaging \\
\hline Pasture pipette bulb & Carl Roth \\
\hline Petri dish 15 mm & BD Biosciences \\
\hline Pipette tips (10-, 100-, 200-, 1000- $\mu$ l) & Sarstedt \\
\hline Plastic wares & Vitlab \\
\hline PMMA cover slip for implant (Self made) & Carl Roth \\
\hline Safety needle (26G) & Sarstedt \\
\hline Syringes ( $2 \mathrm{ml}, 5 \mathrm{ml}, 10 \mathrm{ml}$ ) & BD Biosciences \\
\hline
\end{tabular}




\subsection{3: Chemicals, miscellaneous reagents and commercial kits.}

\begin{tabular}{|c|c|c|}
\hline Name & $\begin{array}{l}\text { Molecular formula } \\
\text { / Abbreviations }\end{array}$ & Company \\
\hline ABC-peroxidase solution & & Vector Labs \\
\hline Agar & & MERCK \\
\hline Albumin bovine serum & & Sigma-Aldrich \\
\hline Ammonium chloride & $\mathrm{NH}_{4} \mathrm{Cl}$ & Sigma-Aldrich \\
\hline Ammonia & & Merck \\
\hline $\begin{array}{l}\text { Bio-Plex Pro }{ }^{\mathrm{TM}} \text { Rat Cytokine } \\
\text { Th1/Th2 Assay \#171-K1002M }\end{array}$ & & Bio-Rad \\
\hline Brain-heart infusion medium & & $\begin{array}{c}\text { Department of Microbiology } \\
\text { Goettingen }\end{array}$ \\
\hline Bromphenol blue & $\mathrm{C}_{19} \mathrm{H}_{9} \mathrm{Br}_{4} \mathrm{NaO}_{5} \mathrm{~S}$ & Fluka \\
\hline Chloral hydrate & $\mathrm{C}_{2} \mathrm{H}_{3} \mathrm{Cl}_{3} \mathrm{O}_{2}$ & Fluka \\
\hline Citric acid & & Merk \\
\hline DAB substrate Kit & & Vector Labs \\
\hline Disodium hydrogen phosphate & $\mathrm{Na}_{2} \mathrm{HPO}_{4} \star 2 \mathrm{H}_{2} \mathrm{O}$ & MERCK \\
\hline Ethyl alcohol 99\% pure & $\mathrm{C}_{2} \mathrm{H}_{5} \mathrm{OH}$ & Chemie-Vertrieb GmbH \\
\hline Ethyl alcohol ultra pure & $\mathrm{C}_{2} \mathrm{H}_{5} \mathrm{OH}$ & Merk \\
\hline Ethylenediaminetetraacetic acid & EDTA & Carl Roth \\
\hline Formalin & & Merck \\
\hline Fluorogold & & Fluorochrome \\
\hline Glacial acetic acid & $\mathrm{C}_{2} \mathrm{H}_{4} \mathrm{O}_{2}$ & Merk \\
\hline Glucose 5\% & & B. Braun \\
\hline Glycerin & $\mathrm{C}_{3} \mathrm{H}_{8} \mathrm{O}_{3}$ & MERCK \\
\hline $\mathrm{HCl}$-ethanol & & Merk \\
\hline $\begin{array}{l}\text { Mycobacterium tuberculosis } \\
\text { (Heat inactivated) (strain H } 37 \\
\text { RA) }\end{array}$ & & Difco Laboratories \\
\hline Hydrochloric acid (32\%) & $\mathrm{HCl}$ & Carl Roth \\
\hline
\end{tabular}




\begin{tabular}{|c|c|c|}
\hline Hydrogen peroxide $30 \%$ & $\mathrm{H}_{2} \mathrm{O}_{2}$ & MERCK \\
\hline Immersion oil for microscopy & & Zeiss \\
\hline Isopropanol & $\mathrm{C}_{3} \mathrm{H}_{8} \mathrm{O}$ & Carl Roth \\
\hline Luria Broth & & Fluka \\
\hline Lithium carbonate & $\mathrm{Li}_{2} \mathrm{CO}_{3}$ & Fluka \\
\hline $\begin{array}{l}\text { Myelin oligodendrocyte } \\
\text { glycoprotein }\end{array}$ & MOG $^{\text {lgd }}$ & $\begin{array}{c}\text { Institute of Neuropathology, } \\
\text { Göttingen }\end{array}$ \\
\hline Mount medium (IF) & & Thermo Scientific \\
\hline Mount medium (IHC) & & Carl Roth \\
\hline Nitric acid & $\mathrm{HNO}_{3}$ & J.T.Baker \\
\hline Paraformaldehyde & PFA & Carl Roth \\
\hline Periodic acid & & Sigma-Aldrich \\
\hline Potassium phosphate & $\mathrm{K}_{2} \mathrm{HPO}_{4}$ & Sigma-Aldrich \\
\hline Potassium chloride & $\mathrm{KCl}$ & MERCK \\
\hline Potassium phosphate & $\mathrm{KH}_{2} \mathrm{PO}_{4}$ & Sigma-Aldrich \\
\hline Schiff's reagent & & Sigma-Aldrich \\
\hline Silver nitrate & $\mathrm{AgNO}_{3}$ & Carl Roth \\
\hline Sodium azide & $\mathrm{N}_{3} \mathrm{Na}$ & Sigma-Aldrich \\
\hline Sodium chloride $(0.9 \%)$ & & B. Braun \\
\hline Sodium citrate & $\mathrm{Na}_{3} \mathrm{C}_{6} \mathrm{H}_{5} \mathrm{O}_{7} * \mathrm{H}_{2} \mathrm{O}$ & MERCK \\
\hline Sodium thiosulfate & $\mathrm{Na}_{2} \mathrm{O}_{3} \mathrm{~S}_{2}$ & Fluka \\
\hline Sodium chloride & $\mathrm{NaCl}$ & MERCK \\
\hline Sodium phosphate dibasic & $\mathrm{Na}_{2} \mathrm{HPO}_{4}$ & Sigma-Aldrich \\
\hline Solvent blue 38 & & Sigma-Aldrich \\
\hline Sucrose & $\mathrm{C}_{12} \mathrm{H}_{22} \mathrm{O}_{11}$ & Fluka \\
\hline Triton X-100 & & Sigma-Aldrich \\
\hline Vaseline & & Lever Faberge \\
\hline
\end{tabular}




\subsection{4: Surgical instruments}

\begin{tabular}{|l|c|}
\hline \multicolumn{1}{|c|}{ Instruments } & Company \\
\hline Cauterizer & Fine Science Tools \\
\hline Forceps & Fine Science Tools \\
\hline Hamilton syringe & Hamilton Messtechnik GmbH \\
\hline Hemostatic clamp & Fine Science Tools \\
\hline Infuse/withdraw syringe pump (KDS 310) & KD Scientific \\
\hline Light source micrcoscope KL 1500 LCD & Foredom \\
\hline Micro motor (FM3545) & Fine Science Tools \\
\hline Rongeurs & Fine Science Tools \\
\hline Scalpel & Fine Science Tools \\
\hline Scissors & Kopf \\
\hline Steriotactic frame (Model 963, Ultra Precise ) & Ethicon \\
\hline Suture & Carl Roth \\
\hline Teflon tube & \\
\hline
\end{tabular}

\subsection{5: Pharmacological products}

\begin{tabular}{|l|c|c|}
\hline Product & Company & Application \\
\hline Ceftriaxone & DesitinArzneimittel GmbH & Wound cream \\
\hline Desitin & B. Braun Melsungen & Infusion (Energy) \\
\hline Glucose 5\% & Abbott & Inhalation Anesthesia \\
\hline Isoflurane & Atarost & Anesthesia \\
\hline Ketamine & Serum-Werk & Pain killer \\
\hline Metapyrin & Pfizer & Painkiller \\
\hline Rimadyl & Albrecht & Anesthesia \\
\hline Xylazine (2\%) & & \\
\hline
\end{tabular}




\subsection{6: List of primary antibodies}

\begin{tabular}{|l|c|c|c|}
\hline Antibody & Conjugate & Company & Application \\
\hline CD134 (OX-40) & FITC & Biolegend & Flow-cytometry \\
\hline CD3 & Alexa fluor 647 & Biolegend & Flow-cytometry \\
\hline CD3 & Biotin & Biozol & IHC \\
\hline CD4 & PE-Cy5 & Biolegend & Flow-cytometry \\
\hline CD45-RA & PE & Biolegend & Flow-cytometry \\
\hline CD8 & PE & Biolegend & Flow-cytometry \\
\hline CD 68 (ED1) & Biotin & AbDserotec & IHC \\
\hline
\end{tabular}

\subsection{6: List of secondary antibodies and purified protein}

\begin{tabular}{|l|c|c|c|}
\hline Antibody (species) & Company & Conjugate & Application \\
\hline Goat anit-mouse IgG & Vector Lab & HRP & IHC \\
\hline Goat anit-rabbit IgG & Vector Lab & HRP & IHC \\
\hline Goat anit-mouse IgM & Vector Lab & HRP & IHC \\
\hline $\begin{array}{l}\text { Streptavidin (for } \\
\text { biotinylated primary Ab }\end{array}$ & Biolegend & PE-Cy5 & Flow-cytometry \\
\hline
\end{tabular}

\subsection{7: List of computer softwares}

\begin{tabular}{|l|c|c|}
\hline Program & Company & Application \\
\hline Adobe Illustrator CS2 & Adobe Systems & Image processing \\
\hline Adobe Photoshop 4.0 & Adobe Systems & Image processing \\
\hline AxioVision & Carl Zeiss & Image processing \\
\hline Definiens Developer XD software & Definiens & Image processing \\
\hline FACSCalibur & BD bioscience & Flowcytometric analysis \\
\hline Bio-Plex Manager 6.0 & Biorad & Cytokine analysis \\
\hline Office Excel & Microsoft office 2007 & Data analysis \\
\hline Prism & GraphPad & Data analysis \\
\hline SPSS & IBM & Data analysis \\
\hline
\end{tabular}




\subsection{8: Buffers and other stock solutions}

\subsubsection{A: Phosphate buffer $10 x$ for 1 liter}

\begin{tabular}{|l|l|l|}
\hline \multicolumn{2}{|l|}{ Chemicals } & Weight \\
\hline 1 & Sodium chloride & $80 \mathrm{~g}$ \\
\hline 2 & Potassium chloride & $2 \mathrm{~g}$ \\
\hline 3 & Disodium hydrogen phosphate & $21.6 \mathrm{~g}$ \\
\hline 4 & Potassium phosphate & $2 \mathrm{~g}$ \\
\hline
\end{tabular}

All the components were mixed in one liter of distilled water and $\mathrm{pH}$ (7.4) was adjusted by using 1 molar $\mathrm{NaOH}$ and $\mathrm{HCl}$.

\subsubsection{B: Citrate buffer}

$6 \mathrm{~g}$ Sodium citrate was added in three liter of water and $\mathrm{pH}$ was adjusted to 6.0.

\subsubsection{C: Developer stock solution for Bielschowsky silver staining}

\begin{tabular}{|l|l|l|}
\hline \multicolumn{2}{|c|}{ Chemicals } & Weight $/$ Volume \\
\hline $\mathbf{1}$ & Formalin & $10 \mathrm{ml}$ \\
\hline $\mathbf{2}$ & Citric acid & $0.25 \mathrm{~g}$ \\
\hline $\mathbf{3}$ & $6 \%$ nitric acid & 2 drops \\
\hline $\mathbf{4}$ & Distilled water & $50 \mathrm{ml}$ \\
\hline
\end{tabular}

\subsubsection{D: RBC lysis buffer}

5.349 grams of ammonium chloride mixed in 1 liter of PBS, pH was adjusted to 7.4.

\subsubsection{E: FACS buffer}

\begin{tabular}{|l|l|l|}
\hline \multicolumn{2}{|l|}{ Chemicals } & Weight $/$ Volume \\
\hline $\mathbf{1}$ & Bovine serum albumin & $10 \mathrm{~g}$ \\
\hline $\mathbf{2}$ & Sodium azide & $1 \mathrm{~g}$ \\
\hline $\mathbf{3}$ & PBS & 1 liter \\
\hline
\end{tabular}




\section{2: Methods}

\subsection{1: Rats}

Female brown Norway rats, 8 to10 weeks of age, weighing between 150-175 grams were used in all experiments. They were obtained from Charles River (Sulzfeld; Germany) and kept under environmentally controlled and pathogen-free conditions. They were given water and standard rodent chow ad libitum. In case animals had severe EAE they were given $5 \mathrm{ml}$ of glucose and sterofudin (1:1) and softened food inside side the cage during the experiment. All experiments involving animal use were performed in accordance with the relevant laws and institutional guidelines of University Medicine Göttingen. These experiments have been approved by the local authorities of Braunschweig, Germany.

\subsection{2: Immunogen}

Recombinant rat MOG ${ }^{\text {Igd }}$, corresponding to the N-terminal sequence of rat MOG (aa 1125), was expressed in E. coli and purified to homogeneity by chelate chromatography (Weissert et al., 1998). The purified protein was then dialyzed against $0.01 \mathrm{M}$ sodium acetate $(\mathrm{pH} 3)$ to obtain a preparation that was stored at $-80^{\circ} \mathrm{C}$ for further use.

\subsection{3: Bacterial inoculum preparation}

\subsubsection{1: Escherichia coli}

The E. coli (Strain $\mathrm{k} 1$, originally isolated from the CSF of a child with meningitis) inoculum was provided by $\mathrm{Dr}$. Roland Nau, Institute of Neuropathology, University Medicine Göttingen. A single colony of $E$. coli was inoculated into Luria Broth medium. Bacteria were harvested after 4 hours of incubation at $37^{\circ} \mathrm{C}$. To determine the exact concentration of bacteria, the inoculum was serially diluted in $0.9 \% \mathrm{NaCl}$ and plated on blood agar plate (Provided by Medical Microbiology, University Medical Center Göttingen) and incubated for $24 \mathrm{~h}$ at $37^{\circ} \mathrm{C}$. Bacterial concentrations were determined by quantitative plating on blood agar plates and aliquots were kept at $-80^{\circ} \mathrm{C}$ until further use. 


\subsubsection{2: Staphylococcus aureus}

The S. aureus strains ATCC 29213 (kindly provided by Raimund Lugert from the University Medicine Göttingen), Newman ATCC 25904 and the extracellular adherence protein (Eap) deficient strain of ATCC 25904, AH12 (kindly provided by Markus Bischoff from the Institute for Medical Microbiology and Hygiene, University of Saarland; Germany) were used in these experiments. A single colony of the respective $S$. aureus strain was inoculated into brain heart infusion medium and incubated for 20 hours at $37^{\circ} \mathrm{C}$ in a shaking incubator. Afterwards, $50 \mu \mathrm{l}$ of the bacterial culture grown for 20 hours were inoculated into $10 \mathrm{ml}$ of fresh brain heart infusion medium and again incubated at $37^{\circ} \mathrm{C}$ in a shaking incubator. Bacteria were harvested after 6 hours of incubation at the time when the bacterial culture reached the log phase of growth. Bacterial concentrations were determined by quantitative plating on blood agar plates, and aliquots were kept at $-80^{\circ} \mathrm{C}$ until further use.

\subsection{4: Retrograde labelling of RGCs}

By retrograde axonal transport, the RGCs were selectively labeled and visualized for fluorescence microscopy. Retrograde labelling of retinal ganglion cells (RGCs) and tissue cage implantations were done during a single anaesthesia 2 to 3 weeks before the MOG-immunization. Rats were anesthetized by intraperitoneal injection of ketamine $(0.95 \mathrm{ml} / \mathrm{kg})$ and xylazine $2 \%(0.25 \mathrm{ml} / \mathrm{kg})$ and positioned in a stereotaxic frame. The skin was incised mediosagittally, and holes were drilled into the skull above each superior colliculus ( $6.8 \mathrm{~mm}$ dorsal and $2 \mathrm{~mm}$ lateral from bregma). $2 \mu \mathrm{l}$ of fluorescent dye fluorogold (5\% in distilled water) were injected into both superior colliculi at a rate of $0.50 \mu \mathrm{l}$ per min. For pain relief, animals received metapyrin in drinking water ( $3 \mathrm{ml} / \mathrm{L}) 2$ days before the start of surgical procedure, which were continued until day 8 of post surgical procedure.

\subsection{5: Tissue cage preparation and implantation}

Rigid polytetrafluoroethylene (Teflon) tissue cages (internal and external diameter of 8 and $10 \mathrm{~mm}$ respectively, length $25 \mathrm{~mm}$ ) perforated by around 100 regularly spaced holes (diameter $1 \mathrm{~mm}$ ) containing two polymethylmethacrylate (PMMA) cover slips per 
tissue cage sealed at both ends with a suture, were used as a foreign body implant. A cutaneous incision in the left flank was made under anaesthesia, and one autoclaved tissue cage was subcutaneously implanted under sterile conditions into each animal. After fluorogold (FG) injection and tissue cage implantation, the wounds were sutured at place of implantation and given $6 \mathrm{ml}$ of a glucose $5 \%$ / Sterofundin solution (1:1) (1/3 intraperitoneally and 2/3 subcutaneously). After tissue cage implantation and colliculus injection animals were treated with Rimadyl 10 $\mu \mathrm{l} / 100 \mathrm{~g} /$ animals (analgesia) followed by metapyrin $(3 \mathrm{ml} / \mathrm{L})$ in drinking water until 8 days post surgical procedure.

\subsection{6: Induction of acute and chronic infection}

To study the effect of acute infection in EAE animals were infected intraperitoneally with $10^{6} \mathrm{CFU}$ of $E$. coli in $200 \mu \mathrm{l}$ of $0.9 \%$ saline. Control rats received an equal volume of saline. The chronic infection with S. aureus was induced by injection of $200 \mu \mathrm{l}$ of bacterial culture diluted in $0.9 \%$ saline containing $2 \times 10^{7}$ colony forming units (CFU) into the agar of the tissue cage. Control animals received $200 \mu \mathrm{l}$ saline into the tissue cage.

\subsection{7: Experimental setup and timeline}

\subsubsection{1: Experimental design for acute infection model}

Number of animals were used in these experiments was 57. Immunized animals were randomly assigned to four different groups. Animals were infected intraperitoneally with E. coli either on day 7 post immunization (early group; Fig 3.2.1.A) or on day 1 of clinical manifestation of EAE (late group; Fig 3.2.1. B). Control rats received an equal volume of saline. To elucidate whether infection could shift the time point of disease onset in MOG-EAE infection was performed during asymptomatic phase of the disease (day 7 post immunization). Number of animals used was 28 ( $n=15$, early infection; $n=13$, control) excluding the animals which died after $E$. coli infection or were sacrificed for ethical reasons.

To reflect the clinical situation in MS patients, animals were infected on day 1 of EAE (late group). Here we addressed the question whether the infection could aggravate the clinical symptoms of EAE. Number of animals used for this experiment 
was 29 ( $n=15$, late infection; $n=14$, control). Animals which either did not show clinical signs of EAE or died after $E$. coli infection were not included in the analysis of late infection group.

\section{A. Early infection group}

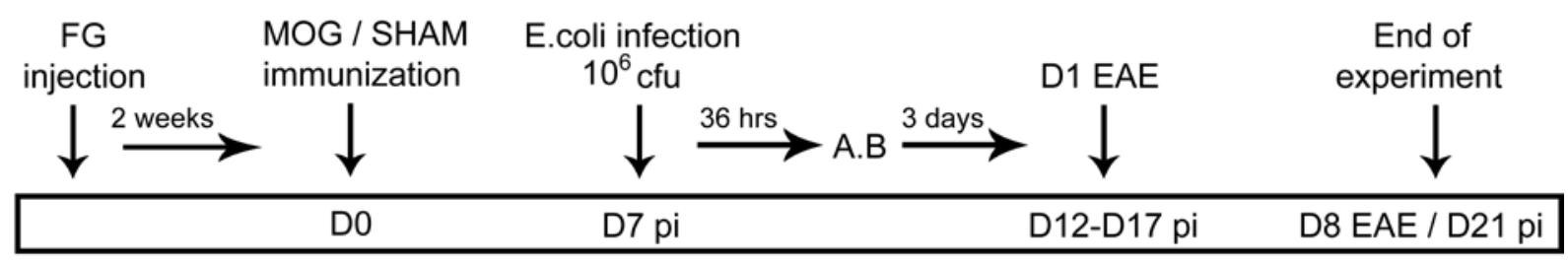

B. Late infection group

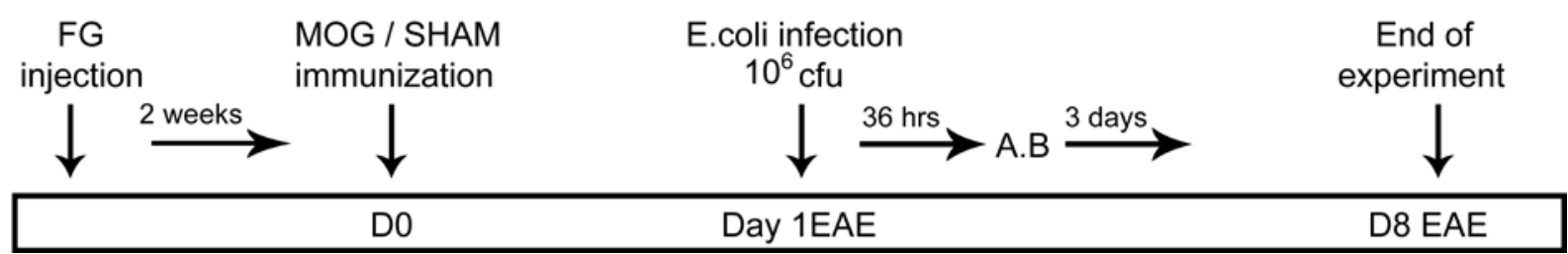

Fig 3.2.1: Experimental design. Fluorogold $(F G)$ injection was performed 2 weeks prior to MOG immunization. A; In early infection group, intraperitoneal injection of $E$. coli or saline was performed on day seven post immunization followed by antibiotic treatment for three consecutive days. Day12 - day17 was the usual time point of clinical onset of disease. Animals were followed until day 8 of EAE or day 21 post immunization in case of clinically healthy animals. $\mathbf{B}$; In late infection group intraperitoneal injection of $E$. coli or saline was performed on the day of clinical manifestation of the disease. Animals were followed until day 8 of EAE. At the end of experiment optic nerve was taken out for histopathological analysis, the retina was flatmounted for quantification of retinal ganglion cells.

To confirm the systemic infection, blood samples were collected from the tail vein under inhalation anaesthesia with isoflurane ( $n=8$ for each group) 24 hours after the infection in the late infection group. Plating of blood samples was performed on blood agar plates under sterile conditions. Animals were followed until day 8 after the clinical manifestation of the disease (EAE d8), or day 21 post immunization in case of absence of EAE symptoms. To ensure the survival of $E$. coli infected animals, antibiotic treatment was started 36 hours after the infection and was followed for three consecutive days at a difference of 12 hours in both the groups. 


\subsubsection{2: Experimental design for chronic infection model and timeline}

Previously Lucet and co-workers established an experimental model for chronic foreign body infection by inoculation of $10^{5}-10^{6} \mathrm{CFU}$ of $\mathrm{S}$. aureus into the implanted tissue cages of male Wistar rats (Lucet et al., 1990). However, due to variation in the strain of the rat (female BN rat), with the similar setup, $10^{9} \mathrm{CFU}$ of $S$. aureus was insufficient to induce a chronic infection.
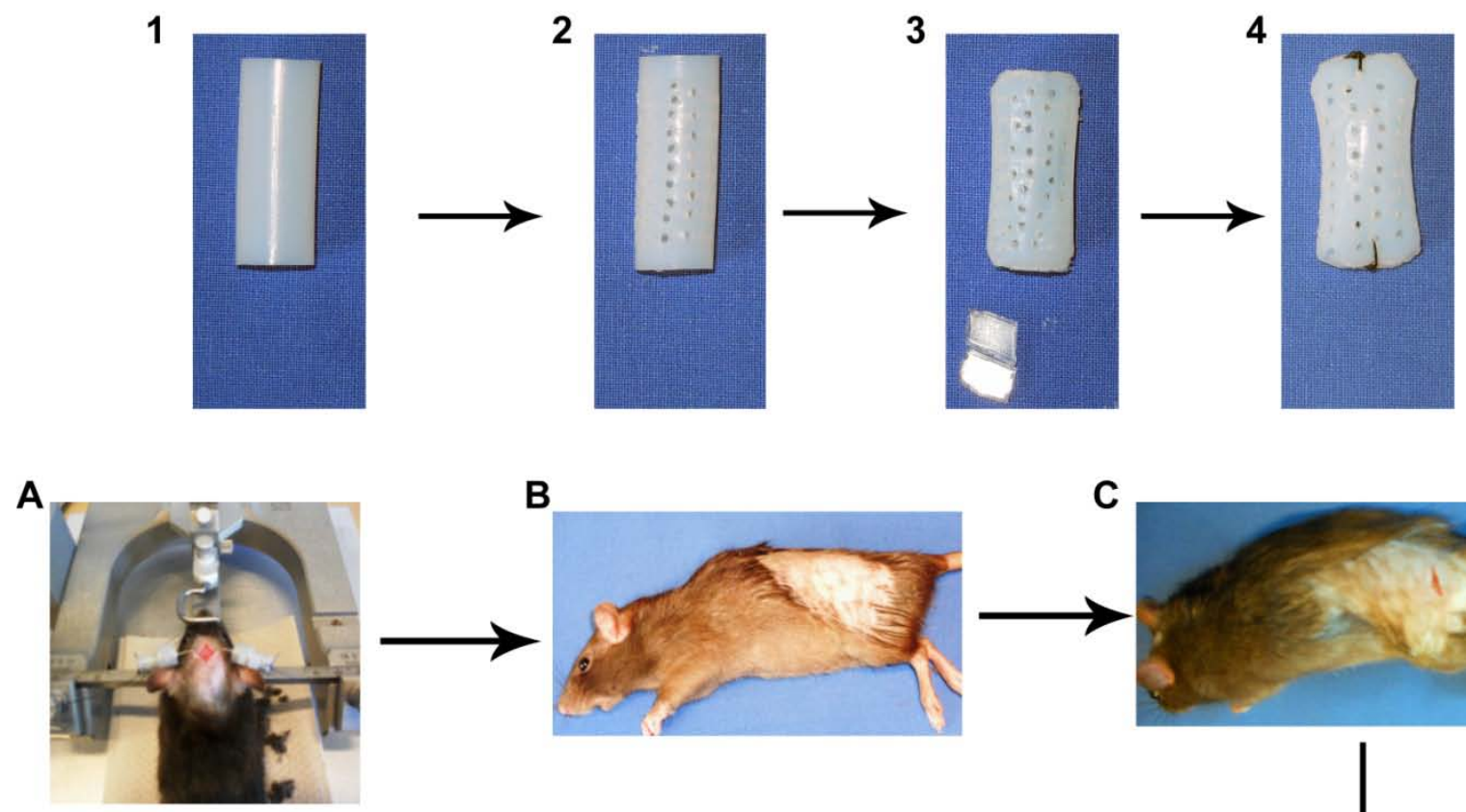

B
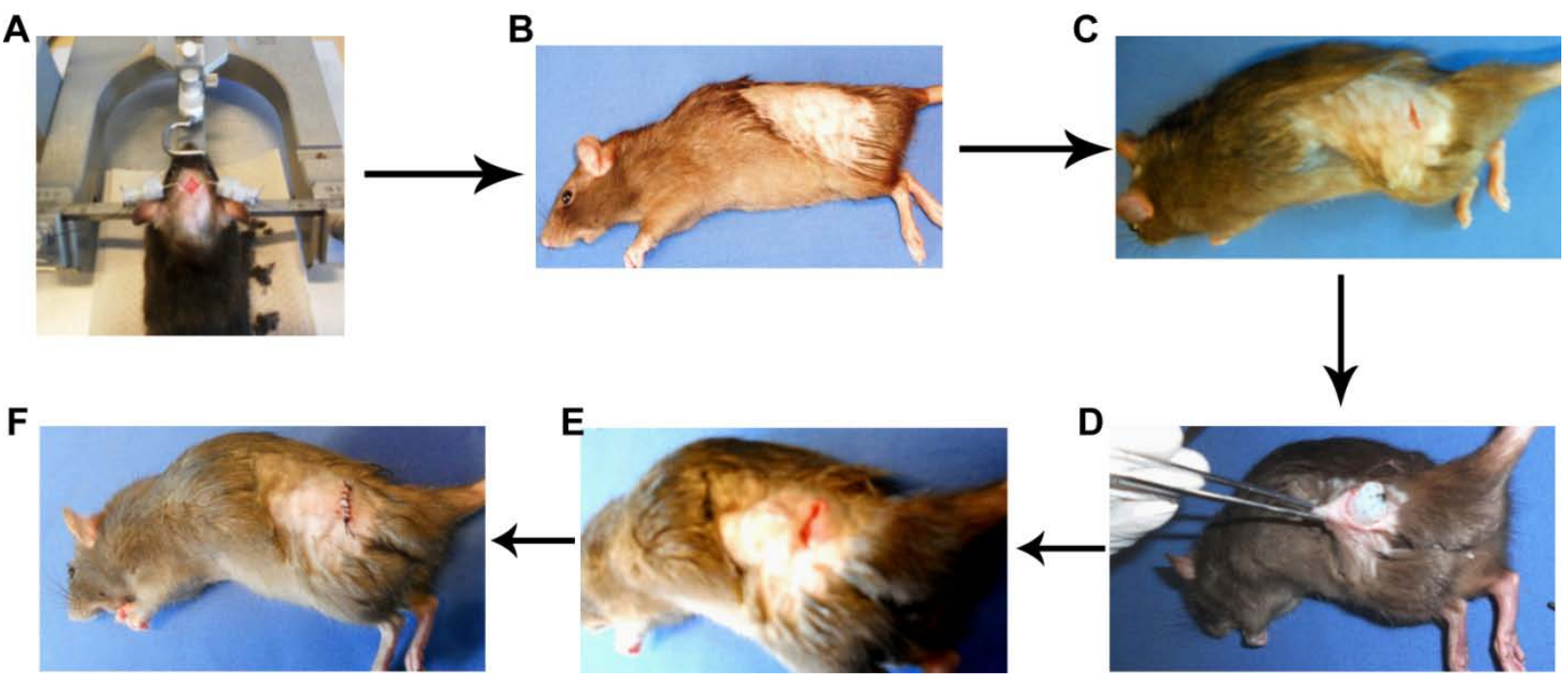

Fig 3.2.2: Experimental setup. Tissue cages were made by using a piece of Teflon plastic (1). Around hundreds of drills were made (2) and 2 PMMA cover slip was placed inside the tissue cage (3) stitched at both ends (4). Animals were anesthetised and fluorogold was injected stereotactically into the superior colliculus (A). Hair was removed (B) and an incision was made in the flank of the animals (C). One tissue cage per animal was placed in the incised region (D). Stitching was done $(\mathbf{F})$ and animals were left 3 weeks for complete recovery. 
Agar is an excellent substrate for the growth of the microorganism and indigestible for many micro-organisms and for animals. Thus to provide more surface area for the attachment of bacteria we injected $400 \mu \mathrm{l}$ of $2.3 \%$ semi-solid agar into the implanted tissue cage using a $27 \mathrm{G}$ needle 6 days prior to the infection (corresponding to 2 days prior to immunization). In such optimized condition animals showed a chronic infection even 3 weeks after inoculation of bacteria in the tissue cages of all the animals. Inoculum dosage was also optimized between inoculum concentrations of $10^{5}$ to $10^{9}$ CFU to establish the chronic infection in this animal model. However, in the absence of agar injection in to the tissue cage, $10^{9} \mathrm{CFU}$ were non-infective. Before the agar injection, tissue cage fluid (TCF) was obtained percutaneously from all animals and checked for the sterility by plating on blood agar plates. Animals were excluded from the experiment if they had contaminated TCF.

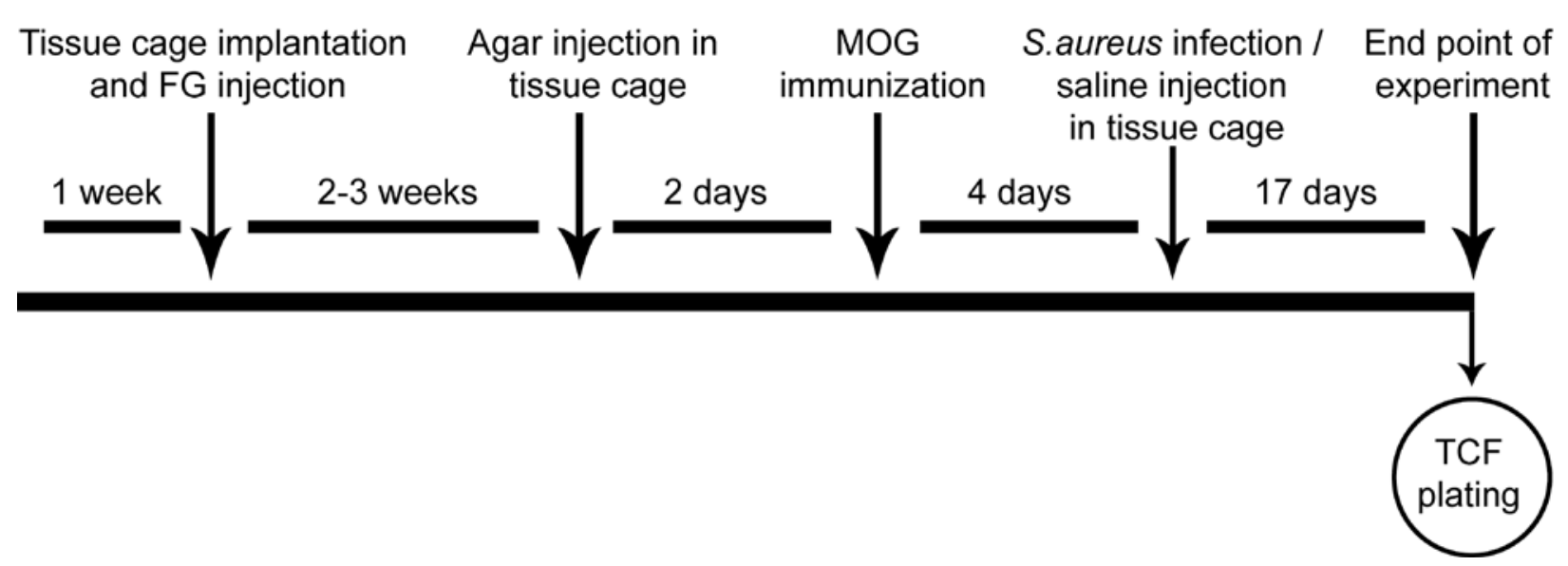

Fig 3.2.3: Experimental timeline. Tissue cage (TC) implantation and fluorogold $(F G)$ injection was done 2 to 3 weeks prior to myelin oligodendrocyte glycoprotein (MOG) immunization. Agar was injected into the TC 2 days before immunization. Injection of Staphylococcus aureus (S. aureus) or saline was performed on day 4 post immunization (pi). Animals were followed until day 8 of experimental autoimmune encephalomyelitis (EAE) or day 21 pi in case of clinically healthy animals. To monitor the infection, blood samples were taken throughout the whole experiment. Tissue was harvested from the optic nerve for histopathological analysis; retina was flat-mounted for quantification of retinal ganglion cells. Tissue cage fluid (TCF) was checked for the presence of bacteria. 
Two days after the agar injection in tissue cage, immunization was done and considered day zero of immunization. Infection was performed on day 4 post immunization (pi). This infection time point was chosen to elucidate whether infection modulates the course of EAE. In the first set of experiment $(n=30)$, one animal group was infected with a wild-type strain of S. aureus (ATCC 29213). The corresponding control group received saline. To prove our hypothesis of anti-inflammatory effect of extracellular adherence protein (Eap), animals $(n=26)$ were again randomized into the three different groups. One group was infected with the wild-type $S$. aureus strain (ATCC 25904). A second group was infected with $S$. aureus strain deficient for Eap (AH12), which is an isogenic mutant of the wild-type Newmen strain of S.aureus (ATCC 25904). The corresponding control group received saline. Animals were followed until day 8 after the clinical manifestation of the disease (EAEd8) or day 21 post immunization (pi) in case of clinically healthy animals. At the end of the experiment, TCF was obtained percutaneously from all (infected and noninfected) animals. TCF was checked for the presence of bacterial colonies by plating on blood agar plates.

\subsection{8: Induction and evaluation of EAE}

Rats were anesthetized by inhalation anaesthesia with isoflurane and injected intradermally at the base of the tail with a total volume of $200 \mu \mathrm{l}$ inoculum, containing $50 \mu \mathrm{g}$ recombinant rat $\mathrm{MOG}^{\mathrm{IgD}}$ (kindly provided by C. Stadelmann, Institute of Neuropathology, University Medicine Göttingen) in saline emulsified (1:1) with complete Freund's adjuvant containing $200 \mu \mathrm{g}$ heat-inactivated Mycobacterium tuberculosis. Rats were scored for clinical signs of EAE and were weighed daily. The signs were scored as follows: grade 0 , no symptoms; grade 0.5 , distal paresis of the tail; grade 1 , complete tail paralysis; grade 1.5, paresis of the tail and mild hind limb paresis; grade 2.0, unilateral severe hind limb paresis; grade 2.5, bilateral severe hind limb paresis; grade 3.0, complete bilateral hind limb paralysis; grade 3.5, complete bilateral hind limb paralysis and paresis of one front limb; grade 4, complete paralysis (tetraplegia), moribund state, or death. 


\subsection{9: Blood leukocyte counts}

To monitor the local application of $S$. aureus in the tissue cage induces the systemic inflammation, leukocyte counts were performed in peripheral blood every second day until day 8 after MOG immunization and then at an interval of 3 days until day 20 pi. Blood samples were taken sublingually after inhalation anaesthesia with isoflurane. A total of $10 \mu \mathrm{l}$ of blood sample was diluted in $190 \mu \mathrm{l}$ of $4 \%$ glacial acetic acid, and leukocyte counting was performed in a Neubauer haemocytometer.

\subsubsection{0: Lymphocytes count after S. aureus infection}

Flow cytometry for lymphocyte counting was performed in blood on day 4 post immunization (pi) prior to infection and 2 days after infection at the time when the leukocyte counts reached their peak. In brief, $100 \mu \mathrm{l}$ blood taken sublingually was collected in $250 \mu \mathrm{l}$ of $5 \mathrm{mM}$ EDTA prepared in isotonic PBS. Samples were washed once with PBS, and RBC lysis buffer (0.1 M ammonium chloride in PBS) was added in each sample. Samples were left at $4^{\circ} \mathrm{C}$ for 10 minutes and washed once with PBS. Samples were divided in two equal parts. In first set of staining a total of $100 \mu$ l of FACS buffer (PBS $+1 \% \mathrm{BSA}+0.1 \% \mathrm{NaN}_{3}$ ) containing florescent tagged anti rat-CD45RA (BioLegend Cat\# 202307) and anti rat-CD3 (BioLegend Cat\# 201408) antibodies along with the beads were added in each samples at a dilution of 1:200. Similarly in the second set of staining a total of $100 \mu$ of FACS buffer containing florescent tagged anti rat-CD3 (BioLegend Cat\# 201408), anti rat-CD8a-PE (BioLegend Cat\# 201705), anti rat-CD134 (OX-40) (BioLegend Cat\# 204503) and anti rat-CD4-AF647 (BioLegend Cat\# 201513) antibodies were added in each samples at the similar dilution of 1:200. Incubation was done for 45 minutes at $4^{\circ} \mathrm{C}$ in the dark. The cells were washed once with PBS and re-suspended in $200 \mu \mathrm{l}$ of FACS buffer prior to flow cytometric analysis. Analysis was performed by four-color flow cytometry using a FACSCalibur and FacScan software (BD; Goettingen; Germany).

\subsubsection{1: Cytokine multiplex assay}

Blood samples were collected at the end of the experiment by puncturing the heart with an 18G syringe before the perfusion of animals and kept on ice for 30 minutes. Serum 
was collected by centrifuging all the samples at 3000 RPM for 5 minutes at $4^{\circ} \mathrm{C}$. Aliquots were kept at $-80^{\circ} \mathrm{C}$ until further use. A Luminex assay kit (Bio-Plex Pro ${ }^{\mathrm{TM}}$ Rat Cytokine Th1/Th2 Assay \#171-K1002M), containing premixed coupled magnetic beads and detection antibodies for detecting 12 rat cytokines (IL-1 alpha, IL-1 beta, IL-2, IL-4, IL-5, IL-6, IL-10, IL-12, IL-13, TNF-alpha, INF-gamma, GM-CSF) was used for measurement of cytokine concentrations in serum samples collected at the end of the experiment. The total number of animals used in this cytokine assay was 25 ( $n=11$ infected with wild-type strain of S.aureus (ATCC 25904); $n=9$ infected with Eapdeficient of S.aureus (AH12); $n=5$ noninfected control). Quantification of cytokines was performed according to the manufacturer's instructions. Measurement was performed by using a Bioplex 200-system based on the Luminex xMAP-technology (Bio-Rad, Hercules, CA, USA). The signal detection occurred using the Bioplex 200-system and the Bioplex Manager Software version 6.0.

\subsubsection{2: Histopathology and immunohistochemistry}

Retinas were removed for counting of RGCs; paraformaldehyde (PFA)-fixed paraffinembedded optic nerves (ONs) were taken for histopathological evaluation. After perfusion of rats with 4\% PFA, ONs were post-fixed in 4\% PFA for $24 \mathrm{~h}$ and embedded in paraffin. All histopathological analysis was performed on paraffin embedded $\mathrm{ON}$ cross sections representing three different levels of an ON.

\subsubsection{1: Staining and quantification of demyelination}

Luxol Fast Blue staining was used to evaluate demyelination on $2 \mu \mathrm{m}$ thick optic nerve s following standard protocol was used for staining

> Sections were deparaffinzed in xylene (2 times for10 minutes), followed by rehydration in descending alcohol series (100\%-, 2 times for 3 minutes; 96\%-, alcohol) for 3 minute each.

$>$ Thereafter, the sections were incubated in LFB solution overnight at $60^{\circ} \mathrm{C}$

> Sections were serially dipped in $90 \%$ alcohol, $0.05 \%$ lithium carbonate solutions, and in $70 \%$ ethanol for 1 minute each. 
> Sections were incubated in $1 \%$ periodic solutions for 5 minutes and followed by a wash in running tap water for 5 minutes and further dipped in distilled water.

$>$ Then sections were incubated in Schiff's reagent for 30 minutes at $4^{\circ} \mathrm{C}$ followed by a washing in running tap water for 5 minutes.

$>$ Sections were counter stained with hematoxylin solution for 7 minutes and dipped in distilled water and $\mathrm{HCl}$-ethanol for few seconds.

$>$ Sections were washed in running tap water for 10 minutes and dipped in distilled water followed by dehydration in ascending ethanol series (50\%-, 70\%-, 90\%-, 96\%- ethanol briefly, 100\%- 2 times for 1 minute, xylene 2 times for 3 minutes).

$>$ Slides were mounted and cover slipped and used for quantification.

\subsubsection{1: Quantification of demyelination}

Photos of vertical sections were taken using an AxioCam MR Microscopy camera (Zeiss, Göttingen; Germany). The images were processed using Zeiss AxioVision 4.2 software to evaluate the demyelinated area as a percentage of the whole ON cross sections.

\subsubsection{2: Axonal count and data evaluation}

To illustrate the axonal counts in the optic nerve, the paraffin sections were stained by the Bielschowsky silver staining. An evaluation was performed on paraffin embedded $0.5 \mu \mathrm{m}$ thick sections of the optic nerve.

Following protocol was used for the staining and data evaluation:

> Sections were deparaffinized in xylene (2 times,10 minutes each), followed by rehydration in descending alcohol series (100\%, 2 times, 3 minutes; 96\%-, 90\%-, 70\%-, 50\%- alcohol) for 3 minute each.

$>$ Thereafter, the sections were incubated for 20 minutes in $20 \%$ silver nitrate solution.

$>$ Dipped in water for a short time. 
Ammonia was added in $20 \%$ silver nitrate solution drop wise until solution turns from bright to dark and turned bright again.

$>$ The optic nerve sections were then transferred back into the silver nitrate ammonium solution and incubated for 15 minutes in the dark.

$>6$ - 8 drops of a developer solution were added.

$>$ Few slides were incubated in silver ammonia solution and looked for dark brown coloration of optic nerve microscopically.

$>$ Slides were washed in distilled water and incubated in $2 \%$ sodium thiosulphate for 2 minutes.

> Slides were washed in running tap water and dehydrated in ascending ethanol series (50\%-, 70\%-, 90\%-, 96\%- ethanol briefly, 100\%- 2 times for 1 minute, xyline 2 times for 3 minute each), mounted and cover slipped.

\subsubsection{1: Quantification of axonal densities in optic nerve}

To quantify the axonal density, $0.5 \mu \mathrm{m}$ thick slices of ONs were stained by Bielschowsky silver impregnation staining to visualize axonal structures. Automated quantification of axonal densities was performed on high magnification pictures (1000x) applying a custom-programmed script in Cognition Network Language based on the Definiens Cognition Network Technology ${ }^{\circledR}$ platform. Briefly, the programmed script first discriminates between tissue and background (no tissue) by spectral difference detection. Subsequently, the area of detected tissue (region of interest, ROI) is calculated and "axonal structures" within this ROI are detected based on their dark black color. To discriminate between nucleoli of inflammatory cells and axons (which both structures are stained black in Bielschowsky silver impregnation) only black structures without a faint brown-stained circumference (nucleus) were further classified as axonal structures. In a next step, multiple axons in direct contact with each other were further split into the corresponding individual axons if the splitting improved the elliptic shape of an axon. Within each area ROI, the total number of axons was calculated. The density of axons in each ON measured in at least nine standardized microscopic fields of each ON. The mean axon density was calculated for each ON and expressed as axons $/ \mathrm{mm}^{2}$. However, in the experiment with acute infection model 
infected with E. coli group and respective control group, axonal density was measured semi-quantitatively by Bielschowsky silver impregnation staining on $2 \mu \mathrm{m}$ thick vertical section of ONs in three standardized microscopic field per. In brief axonal density was determined in vertical sections of the ON by point sampling using a 25-point Zeiss eye piece. The number of points crossing axons was measured as a fraction of the total number of points of the stereological grid.

\subsubsection{3: Immunohistochemistry}

Immunohistochemistry was performed in serial sections of paraffin-embedded ONs. ED1 macrophages/ activated microglia and CD3-positive cells were detected in cross sections using biotin-avidin detection. Spleen sections served as positive control for ED1 staining. The following standard protocol was followed for immunohistochemical analysis;

$>$ The paraffin embedded slides were deparaffinised by placing twice in xylol for 10 minute each.

$>$ Sections were rehydrated in descending ethanol concentration for 3 minute each (100\% 2 times, 96\%-, 90\%-, 70\%-, 50\%- ethanol) followed by placing them in water.

$>$ Antigen retrieval was performed by heating the slides into microwave in citrate buffer for 10 minutes at 800 watt and 5 minutes for 560 watt.

$>$ Slides were allowed to cool down for 20 minutes at room temperature.

$>$ Quenching of endogenous peroxidise was done by dipping the slides into 3\% $\mathrm{H}_{2} \mathrm{O}_{2}$ for 10 minutes at room temperature.

$>$ Slides were washed with PBS (3 times for 5 minutes).

$>$ Blocking was done for $1 \mathrm{~h}$ at room temperature with 10\% serum (ED1) or in $10 \%$ serum+ $2 \%$ BSA (CD3).

> Primary antibodies were applied in appropriate dilutions (1/500 for ED1 and $1 / 200$ for CD3).

$>$ Slides were incubated at $4^{0} \mathrm{C}$ (For ED1, 12-16 h; for CD3, 20-24 h). 
D Washing was done with PBS (3 times for 5 minutes) followed by incubation with secondary antibody $1 \mathrm{~h}$ at room temperature.

> Washing with PBS (3 times for 5 minutes) was done.

$>$ Sections were incubated with VECTASTAIN $® A B C$ Kit (Vector Burlingame; USA) for $1 \mathrm{~h}$ at RT followed by washing with PBS (3 times for 5 minutes).

$>$ Development was done by using DABI kit.

\subsubsection{1: Quantification of ED1 positive cells}

The evaluation of ED1 positive cells was performed using the following score: 0, no labelled cells; 1, a few ED1 positive cells (number countable) in at least one of the three cross sections of an ON; 2 infiltration of less than 10\% of the ON area with ED1 positive cells (number not countable) in at least one of the three cross sections of an ON; 3 , infiltration of $10-50 \%$ of the ON area with labelled ED1 positive cells in at least one of the three cross sections of an ON; 4 , infiltration of $80 \%$ of the ON area; 5 , infiltration of more than $80 \%$ of the ON area in at least one of the three cross sections of an ON.

\subsubsection{2: Quantification of T-cell infiltrates in the optic nerve}

CD3 staining for assessment of infiltration of CD3 positive cells across the BBB was performed on $0.5 \mu \mathrm{m}$ thick slices of paraffin-embedded ONs using anti-CD3 antibody. CD3 positive cells were detected by biotin-avidin detection. Spleen sections served as positive control for CD3 staining. To quantify T-cell infiltrates in the ON, we used tissue studio that is based on Cognition Network Language (Definiens Cognition Network Technology; Definiens Developer XD software, Munich; Germany) In brief, CD3 stained sections were scanned using the 3D Histech 250 Flash slide scanner at 200 fold magnification. The area of interest (optic nerve) was first detected from the background by the colour spectrum. After segmentation, nuclei and CD3-positive cytoplasm were discriminated. Within each area of interest, the total number of CD3-positive cells was calculated and expressed as cells $/ \mathrm{mm}^{2}$. 


\subsubsection{3: Quantification of RGC density}

Eyes were enucleated from the rat before perfusion and kept in PFA for 2 minutes and transferred to PBS. An incision was made by using a 23G syringe on the junction of Sclera and the cornea. Frontal part was removed along with the lens. Then vitreous humour present on the retinal surface was taken out carefully. Retinae were dissected, flat-mounted on glass slides, and examined by fluorescence microscopy (Zeiss, Axioplan 2) using a DAPI filter (315/395 nm). Retinal ganglion cell densities were determined by counting labelled cells in three areas $\left(62,500 \mu \mathrm{m}^{2}\right.$ each) per retinal quadrant at three different eccentricities of $1 / 6,3 / 6$, and $5 / 6$ of the retinal radius. Cell counts were performed following a blinded protocol.

\subsubsection{4: Statistical analysis}

Data are presented as means \pm SEM. Student's t-test was used to analyze differences between two animal groups. Multiple comparisons were statistically evaluated by oneway ANOVA using Prism 5 (GraphPad software). Mann-Whitney $U$ test was used for the analysis of the EAE score (SPSS, IBM). Data presented as means \pm S.E.M. of the daily score. A p-value $(P)$ of $<0.05$ was considered to be statistically significant and indicated as follows *, $P<0.05$; ${ }^{\star *}, P<0.01$; ${ }^{* \star}, P<0.001$. "n" indicate the number of animals. However, for RGCs counting $\mathrm{n}$ indicate the number of eyes used for counting. 


\section{RESULTS}

\section{1: Acute E. coli infection and EAE}

Evidences from experimental and clinical studies indicated that that the progression of multiple sclerosis (MS) is influenced by systemic infection caused by bacteria. In most of the previous experimental studies only bacterial components were used to mimic the situation of the bacterial infection that probably might not be the real clinical condition. Therefore to address the impact of a viable bacterial infection in MS, we infected an animal model of MS with $E$. coli, which causes the vast majority of urinary tract infections. Moreover, we infected the animals at two different time points to address the question that does the time course of infection have any impact on the onset of the clinical disease symptoms, when infection was performed in the preclinical phase of disease (day 7 post imunization) "early infection group" and on the severity and aggravation of clinical disease course, when the infection was performed on day 1 of clinical manifestation of the disease (day 1EAE) "late infection group".

\subsection{1: Intraperitoneal infection with $E$. coli induces systemic inflammation}

The common notion is that a bacterial infection induces changes termed as a sickness behavior, which reflects in animals in form of weight loss, rise in body temperature, increase in heartbeat counts, sepsis and sometimes as the death of the animal.

In the early infection group, the animals were infected with $E$. coli on day 7 after MOG-immunization, whereas late infection group reflects the condition when infection was induced on the day of clinical onset of disease (day 1EAE). In the early infection group (Fig; 4.1.1 A) the animals infected with $E$. coli $(n=15)$ showed significant weight loss after the infection compared to the control group $(n=13 ; P<0.01)$. This loss in weight of animals indicated a general sign of sickness behavior. These animals recovered their weight loss after antibiotic treatment. In contrast, in the late infection group (infected on day 1 EAE; Fig 4.1.1 B) the animals infected with $E$. coli $(n=15)$ did not show any significant difference in weight loss compared to the control group $(n=14$; $B ; P=0.69)$. 

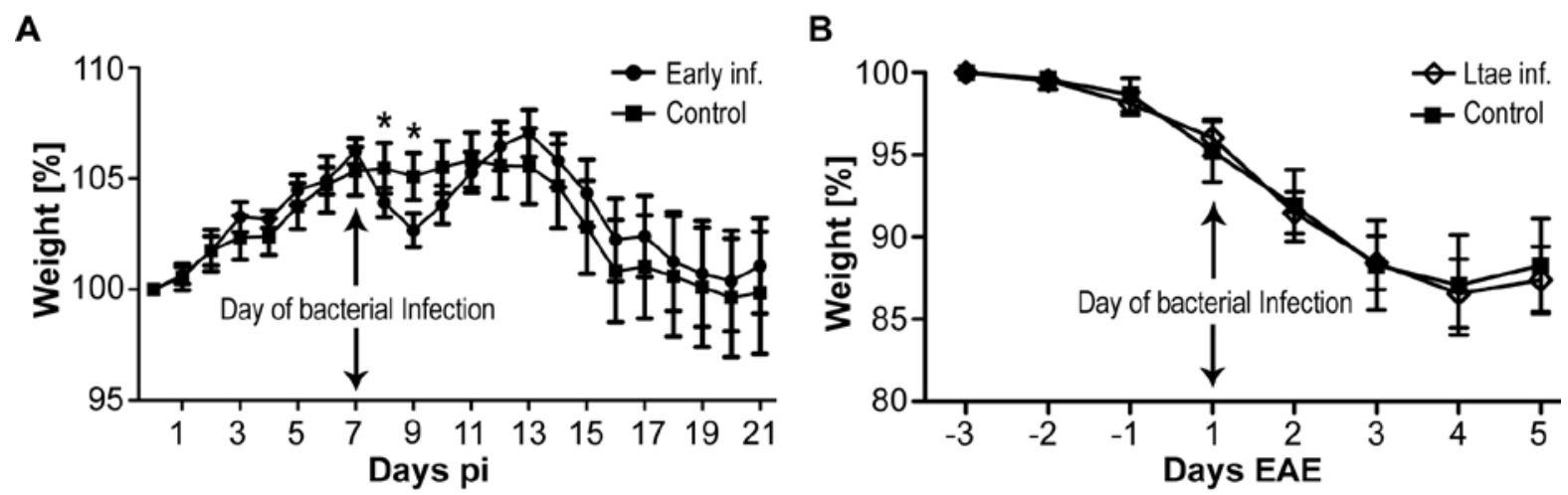

Fig 4.1.1: Weight loss: Animals infected with E. coli on day 7 post immunization $(A)$ showed a significant weight loss compared to the control group. No significant weight loss was observed in late infection group (B) compared to control.

The loss in the weight was probably due to the fact that during the clinical disease course of EAE both infected and control groups lost their weight rapidly. Therefore, to confirm the systemic infection in a late infection group of animals, 24 hours after the infection, blood samples were collected from the tail vein of animals infected with $E$. coli and from the respective control group ( $n=8$ in each group) under sterile conditions. Plating of blood samples was performed on blood agar plates, which showed a mean bacterial count $\left(\log _{10} 3.64 \pm 3.32 ; P=0.04\right)$ only in the animals infected with E. coli. No bacterial counts were detected in saline treated control animals. The bacterial detection limit was $100 \mathrm{CFU} / \mathrm{ml}$. We also observed a mortality rate around $20 \%$ due to $E$. coli infection in both early and late infection group. However, the data from these animals were not included in the analysis of the results.

\subsection{2: The influence of $E$. coli infection on clinical course of MOG-EAE}

The "early infection" group infected animals did not show any significant difference in the mean cumulative clinical score in comparison to the control animals $(0.9 \pm 0.35, n$ $=15$ vs. $1.038 \pm 0.39, \mathrm{n}=13 ; P=0.8 ;$ Fig 4.1.2 A). Also the onset of disease was similar in both the groups (13 days post immunization in the infection and 12 days post immunization in the control group). 
A

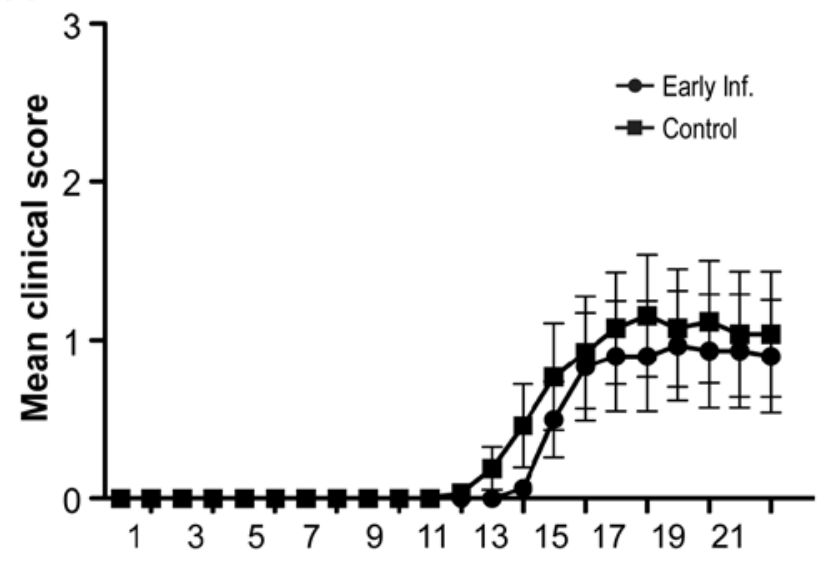

B

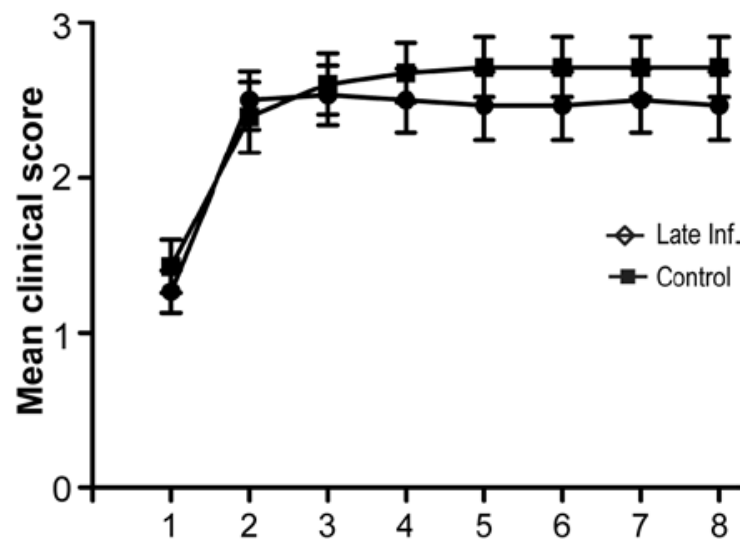

Fig 4.1.2: Mean clinical score. Infection with E. coli on day 7 post immunization (early infection group; A) had no impact on the time of clinical onset or severity of the disease in MOG-EAE. Infection with $E$. coli on day 1 EAE (late infection group, B) did not show any significant impact on the severity of the disease compared to the control group. Data are shown as means \pm S.E.M. of the daily score.

In the second set of experiments, infection with $E$. coli was induced on the day when the animals showed first clinical symptoms (late infection group). E. coli infection did not substantially modulate the course of EAE. Here, the mean cumulative score was not significantly differed between the group infected with $E$. coli and the respective control group ( $2.47 \pm 0.22 \mathrm{n}=15$ vs. $2.71 \pm 0.19 \mathrm{n}=14 ; P=0.51 ;$ Fig $4.1 .2 \mathrm{~B})$ at the end of the experiment.

\subsection{3: Infection with E. coli and neuropathological damage of the optic nerve}

Animals were sacrificed on either day 8 of clinical manifestation of disease or at day 21 after immunization in the absence of clinically manifest disease. Histopathological analysis was performed on paraffin embedded optic nerve s. We determined the extent of demyelination of the ONs by Luxol Fast Blue staining. In the early infection group we did not observe any significant difference in demyelination of $E$. coli infected animals (62.90 \pm 9.43\%; Fig 4.1.3 A, G; $n=15)$ compared to control group $(79.18 \pm 8.06 \% ; P=$ 0.20; Fig 4.1.3 B, $G ; n=13$ ). Analysis of inflammatory infiltrates by ED1 staining revealed no significant difference in the animals infected with $E$. coli on day 7 post immunization (2.84 \pm 0.39; Fig 4.1.3 C, H; $\mathrm{n}=15$ ) compared to the saline treated control 
animals (3.45 $\pm 0.3 ; P=0.50$; Fig 4.1.3 $\mathrm{D}, \mathrm{H} ; \mathrm{n}=13$ ). Bielschowsky silver impregnation was performed to access the axonal loss of the ON. At day 8 of MOG-EAE, we observed a loss of ( $66 \pm 7.64 \%$; Fig 4.1.3 E, l; $n=15$ ) in the animals infected with E. coli. In the control group, the axonal loss did not differ significantly $(82.19 \pm 5.69 \% ; P=0.1$; Fig 4.1.3 $F, I n=13)$.

A

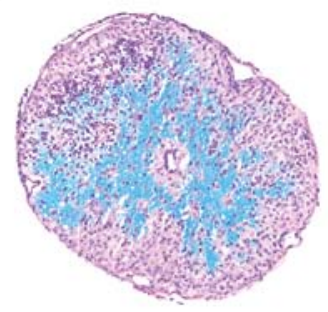

C

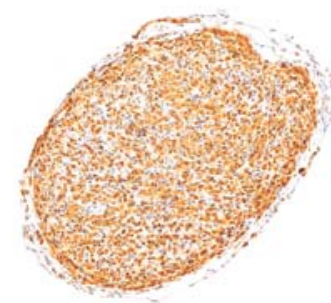

E

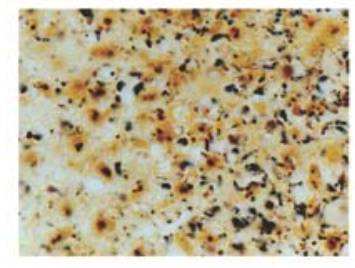

B

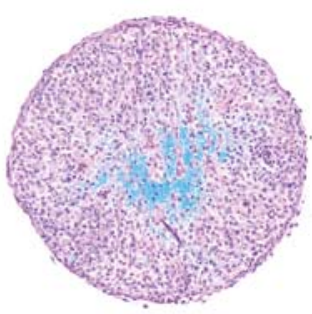

D

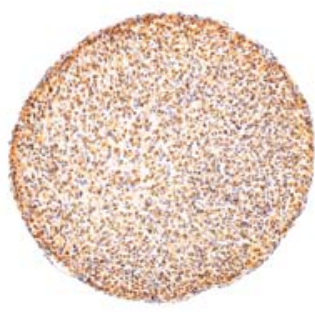

$\mathbf{F}$

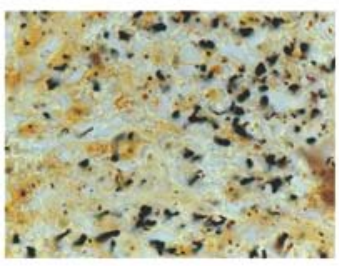

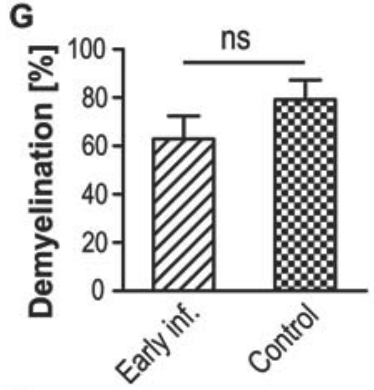

H
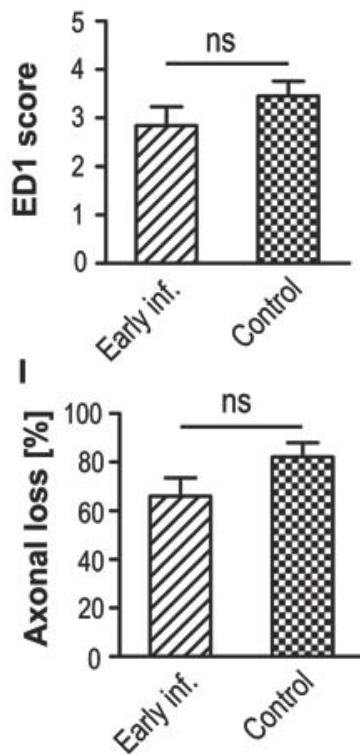

Fig 4.1.3: Optic nerve (ON) histopathology for early group: A and B: Representative Luxol Fast Blue-stained s of ONs of an animal infected with $E$. coli (A) and an animal from respective control group (B). Representative examples for number of infiltrating ED1 macrophages / activated microglia detected in the $\mathrm{ON}$ of an E. coli infected animal (C) and an animal from noninfected control group (D). E and F: Bielschowsky's silver impregnation of an ON revealed no differences in axonal densities of MOG immunized animal infected on day 7 post immunization (E) compared to noninfected control animal (F). G, H and I represent the quantitative data for demyelination, inflammatory infiltrates and for axonal counts in both E. coli infected and control group. 
In the experiment performed with "late infection" a similar scenario was observed. Here the extent of demyelination (77.06 $\pm 5.77 \%$; $n=15$; for infected group; $72.87 \pm 6.46 \%$; $\mathrm{n}=14$; for the control group; $P=0.63$; Fig 4.1.4, A) and number of inflammatory infiltrates (3.944 $\pm 0.24 ; n=15$; for infected group; $3.827 \pm 0.26 ; n=14$; for the control group; $P=0.74$; Fig 4.1.4, B) did not show any significant difference between the group infected with E. coli and the saline treated control group. Moreover, we did not find any effect of $E$. coli infection on axonal loss as quantified by Bielschowsky silver impregnation (71.84 $\pm 5.03 \%$; $n=15$; for infected group and $70.07 \pm 5.39 \%$; $n=14$; for the control group; $P=0.81 ;$ Fig 4.1.4, C).

A

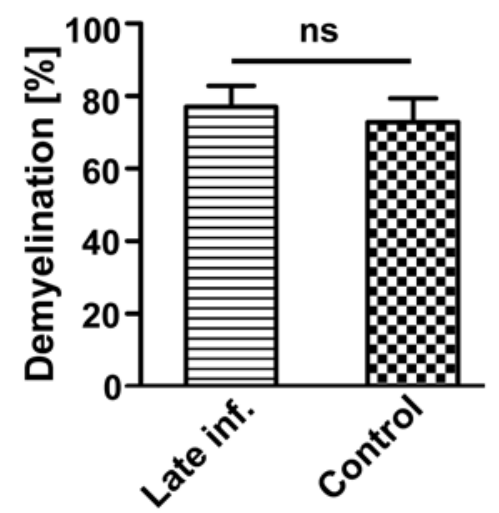

B

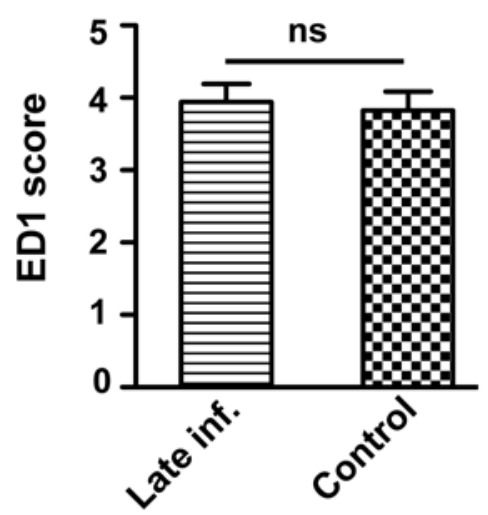

C

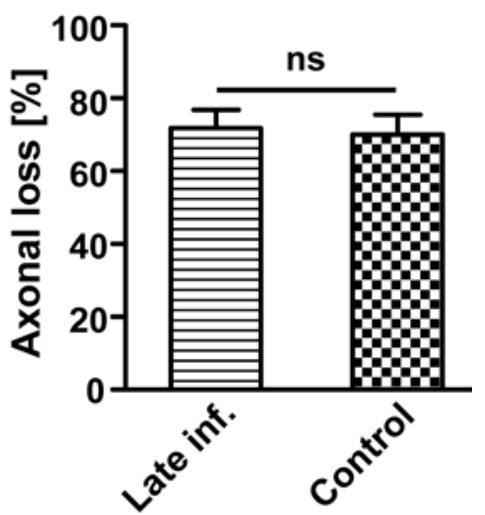

Fig 4.1.4: Optic nerve (ON) histopathology for late infection group: No significant difference was observed in quantitative data analysis for demyelination (A), inflammatory infiltrates (B) and for axonal counts (C) between the groups infected with $E$. coli and the respective control.

In summary these results from the histopathological analysis performed on optic nerve cross sections indicated that the $E$. coli infection did not have any influence on demyelination, infiltration of inflammatory cells or on axonal damage.

\subsection{4: Impact of $E$. coli infection on loss of RGCs}

It is presumed that bacterial infections lead to enhanced neurodegeneration. Therefore to evaluate the impact of infection on neuronal damage in MOG-EAE after $E$. coli infection, numbers of surviving RGCs were counted on fluorogold labeled retinal flat mount. In the early infection group (Fig 4.1.5 A; n=25 each) RGCs density did not reveal 
any significant difference between $E$. coli infected group $\left(1411 \pm 87.57 / \mathrm{mm}^{2}\right)$ compared to corresponding control group (1248 $\left.\pm 68.99 / \mathrm{mm}^{2} ; P=0.15\right)$.

A

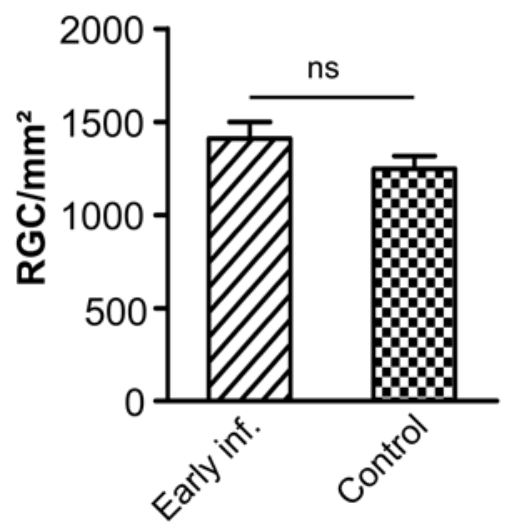

B

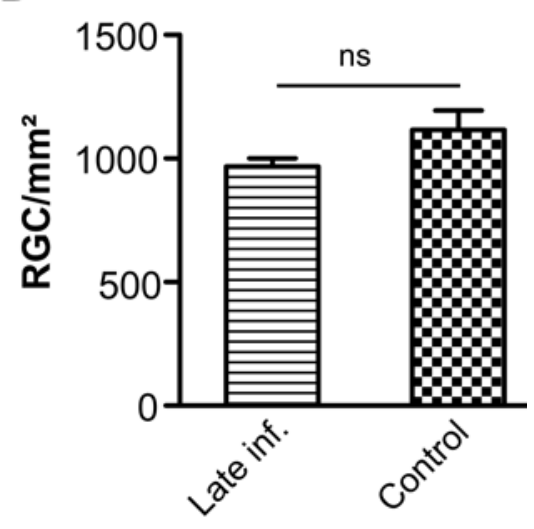

Fig 4.1.5: RGCs counting: No significant differences were observed in a number of surviving retinal ganglion cells (RGCs) of the animals infected with $E$. coli either in preclinical phase of disease (A) or in clinical phase of disease (B).

Furthermore, to know the impact of infection during the clinical course of the disease the RGCs counting performed in the late infection group of animals which (Fig 4.1.5 B; n=16 each group) did not reveal any significant difference between the group infected with $E$. coli $\left(967 \pm 33 / \mathrm{mm}^{2}\right)$ compared to the corresponding control group (1116 $\left.\pm 78 / \mathrm{mm}^{2}\right)$. However, the group infected with $E$. coli on day of onset of the disease showed a tendency towards more neuronal cell loss compared to the respective control group $(P=$ 0.08). 


\section{2: Chronic S. aureus infection and EAE}

Several acute infection models have been used to study the impact of infection on the course of EAE and mostly showed a deleterious effect on severity of disease. However, most of these studies also used an immediate antibiotic treatment to ensure the survival of animals after the infection. These situations mask the impact of the infection alone since the modulatory role of these antibiotics in the progression of EAE has been proven (Melzer et al., 2008; Mor and Cohen, 2013). Moreover, there is no direct evidence of $S$. aureus or any other chronic infection causes modulation of the EAE. In this study to ensure the chronic infection, to achieve this tissue cage was implanted under the skin and infected with $S$. aureus which leads to the chronic infectious condition in this animal model. No antibiotic treatment was performed during the course of the experiment.

\subsection{1: Local infection with S. aureus leads to persistent systemic inflammation}

The animals were infected with $S$. aureus on day 4 post immunization (pi) into the previously implanted tissue cage. After infection we did not observe any significant weight loss in the animal infected with S. aureus (Fig 4.2.1). However, animals from the control group lost their weight once they start showing clinical sign of EAE.

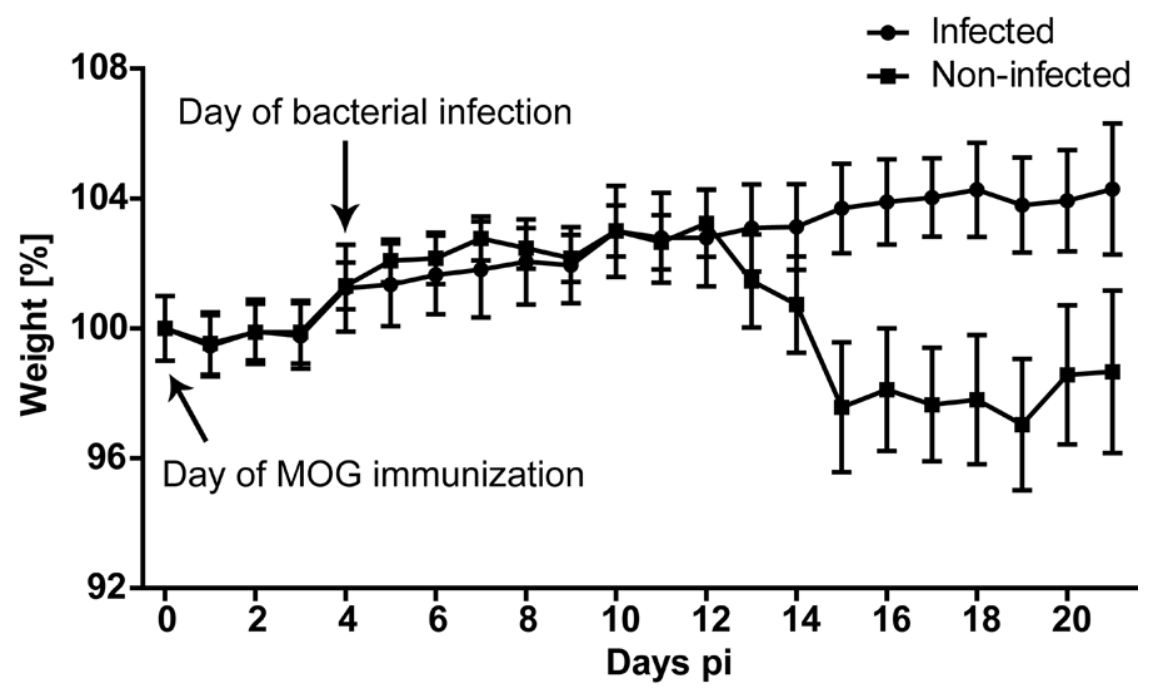

Fig 4.2.1: Weight loss: After infection no significant weight loss was observed in animals infected with S. aureus. 
Therefore to confirm the persistent local infection in the animals we collected TCF (tissue cage fluid) from all animals under sterile conditions at the end of the experiment and plated the TCF on the blood agar plates. TCF obtained from infected animals contained a high number of bacteria ( $S$. aureus), whereas no bacterial growth was observed in TCF from noninfected control animals. Due to the semi-solid consistency of TCF, which is probably due to the presence of agar, dilutions for a more exact determination of bacterial numbers were not possible. In this animal model the inoculation with $10^{7} \mathrm{CFU}$ was optimal to induce the chronic infection.

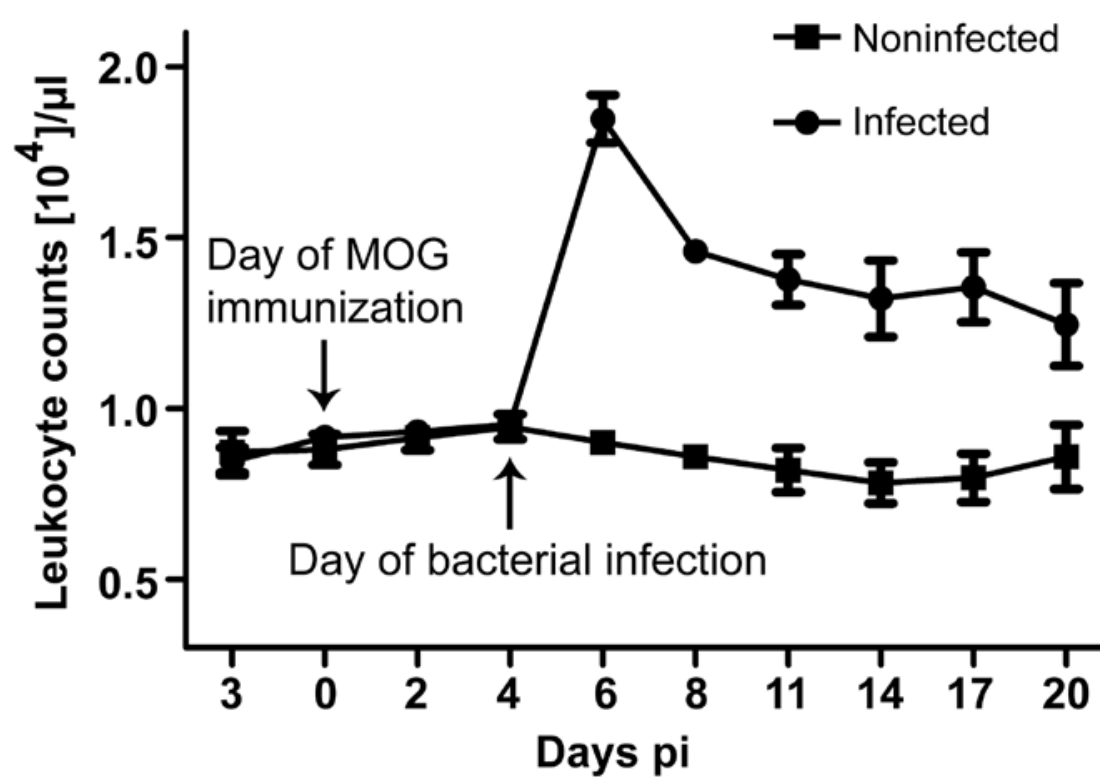

Fig 4.2.2: Blood leukocyte counts: Leukocyte counts were performed in peripheral blood of the animals infected with $S$. aureus wild type and noninfected myelin oligodendrocyte glycoprotein (MOG)-immunized control animals. The leukocyte counts were found to be significantly higher in the group infected with S. aureus.

During the course of the experiment we did not observe the presence of bacteria in the peripheral blood of animals. Therefore to monitor the local application of bacteria induced systemic inflammation, blood leukocyte counts were performed in peripheral blood samples until day 20 pi (corresponding to day 16 post infection). Here, we observed that the leukocyte counts in the $S$. aureus infected animals $(18475 \pm 685 / \mu \mathrm{l}$; $\mathrm{n}=12$; Fig 4.2.2) were significantly elevated two days after infection compared to noninfected control animals (9000 $\pm 305 / \mu \mathrm{l} ; \mathrm{n}=11 ; P<0.001$; Fig 4.2.2). Afterwards, 
leukocyte counts decreased in the $S$. aureus infected animals but, remained significantly elevated compared to the control animals until day 20 pi $(12457 \pm 1219 / \mu \mathrm{l}$ versus $8583 \pm 936 / \mu \mathrm{l}, P=0.03$; Fig 4.2.2). This increase in leukocyte counts in animals after $S$. aureus infection confirmed a strong chronic systemic inflammation.

\subsection{2: Infection with S. aureus enhanced lymphocyte counts in peripheral blood}

Flow cytometry was performed to detect the lymphocyte counts in peripheral blood on day 4 post immunization (pi) (directly prior to infection) and day 6 post immunization (corresponding to day 2 after the infection).

B-cell counts were significantly increased in the $S$. aureus infected group $(67 \pm$ 10.55\%; $P=0.002$; Fig 4.2.3 B, D; $\mathrm{E} ; \mathrm{n}=4$ ) on day 6 pi compared to day 4 pi. In contrast, no significant change in B-cell counts was observed in noninfected animals on day 6 pi compared to day 4 pi (Fig 4.2.3 A, C; E). T-cell counts increased significantly in both the groups on day 6 pi compared to day 4 pi, however, the increase was more prominent in infected than in noninfected animals (117 $\pm 19.38 \%$ (Fig 4.2.3 D, F; versus $45 \pm 8.32 \% ; P=0.013 ;$ Fig 4.2.3 C; $F ; n=4$ ).

Subsequently, T cell subpopulations were also counted and we observed a significant increase in Th- and Tc-cell count in the animals infected with $S$. aureus on day 6 pi compared to day 4 pi (Fig 4.2.3 G, H). However, the Th-cell count was also increased significantly in the control group (Fig 4.2.3 G) and showed an increasing trend in Tc-cell counts (Fig 4.2.3 H). Moreover, CD134 (Ox40) an activation marker for T-cells counts showed an increasing trend in both the infected and noninfected group (Fig 4.2.3 I) on day 6 pi corresponding to day 2 post infection. These results indicated that infection with $S$. aureus induces both B-cell and T-cell response in the periphery without any significant difference in the number of activated T-cell counts. 
A

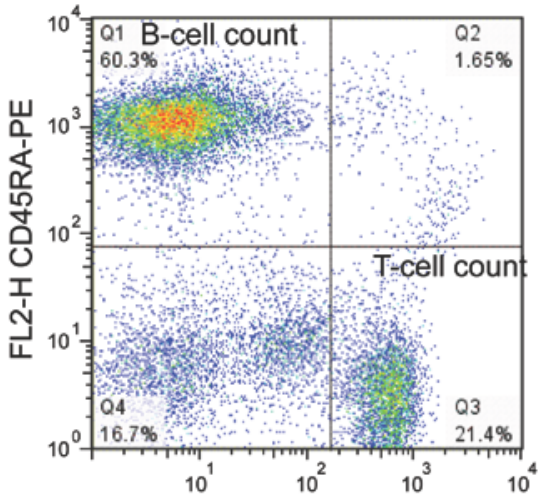

C

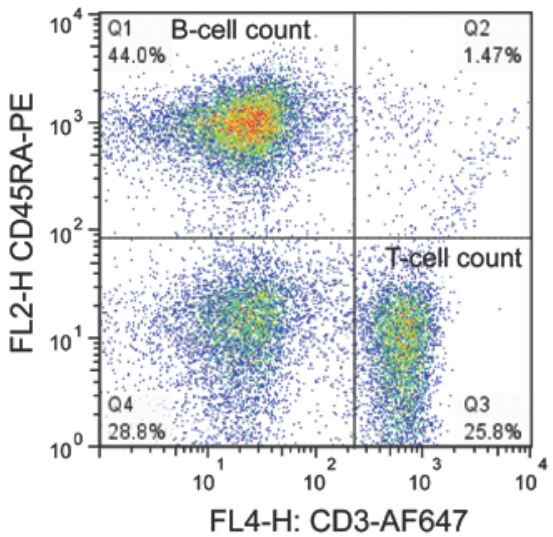

G

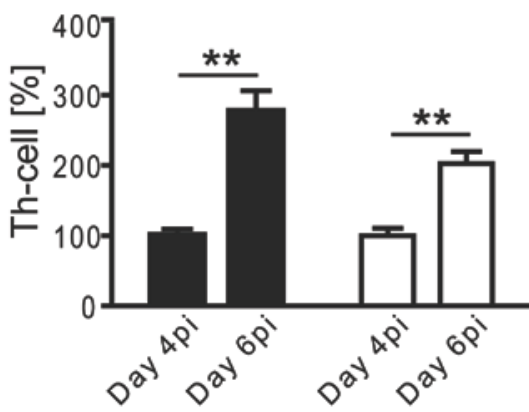

B

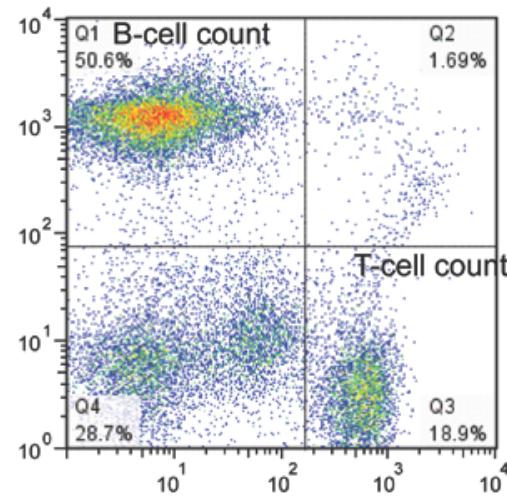

D

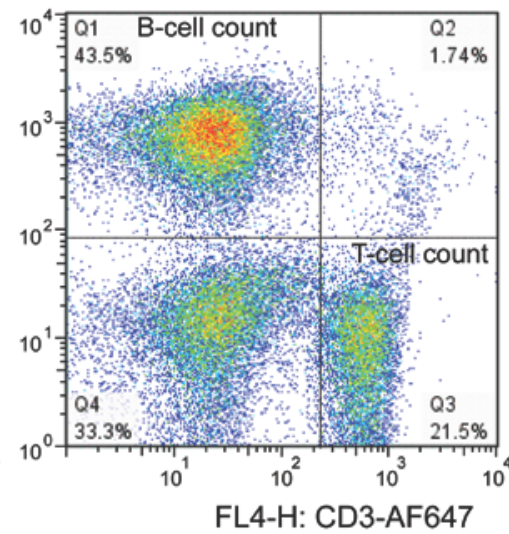

H

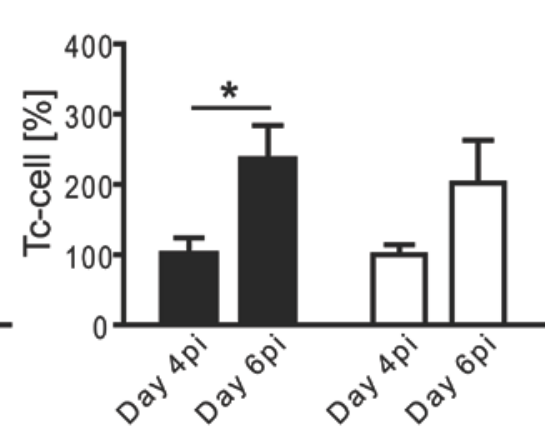

E
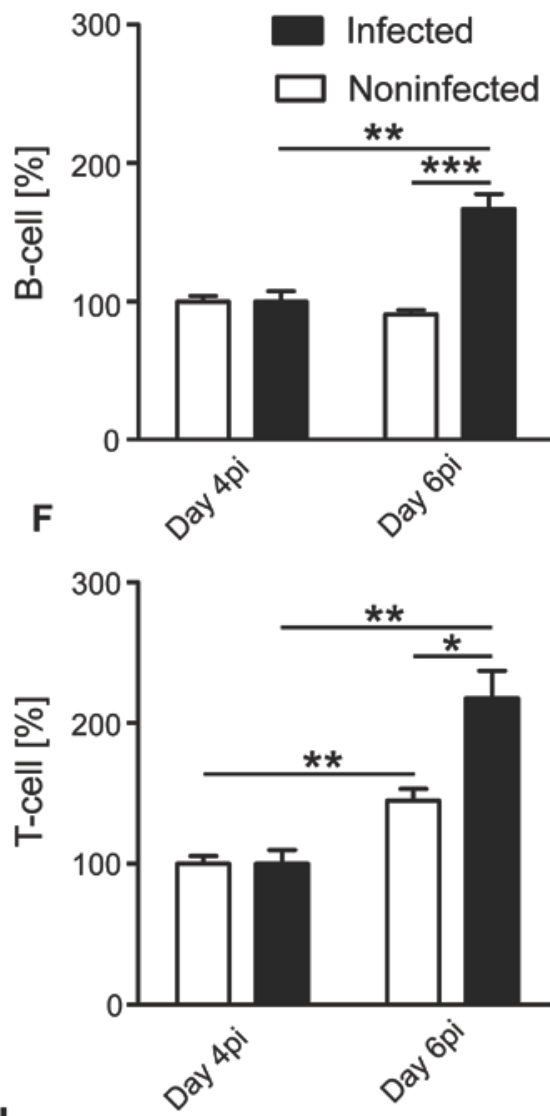

1

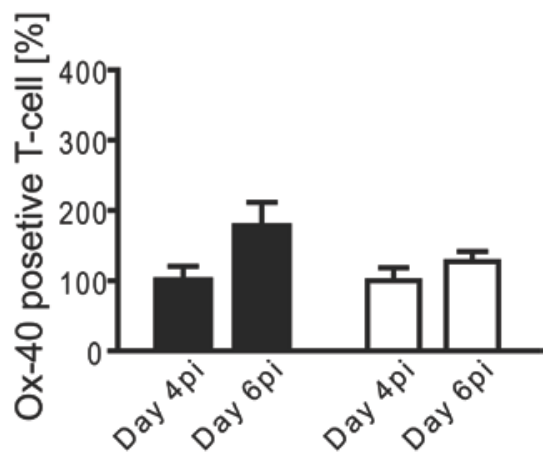

Fig 4.2.3: Flow cytometry: Flow cytometry was performed in peripheral blood to assess lymphocyte populations in animals infected with $S$. aureus (B, day 4 ; $\mathbf{D}$, day 6 ) and noninfected control group (A, day 4; C, day 6) on day 4 post immunization (prior to infection) and on day 6 post immunization (corresponding to day 2 post infection). $\mathbf{E}$ and $\mathbf{F}$ represent the quantitative data for B- and T-cell counts, which were substantially increased in infected animals two days after infection. G, $\mathbf{H}$ and I represent the quantitative data for Th-, Tc- and the number of activated T-cells on day 6 compared to day 4 post immunization. 


\subsection{3: Chronic S. aureus infection and disease activity in MOG-EAE}

There is a possibility that infection may interfere with the immunization process. Therefore to avoid interference with the initiation of the autoimmune response animals were infected with $S$. aureus on day 4 after immunization

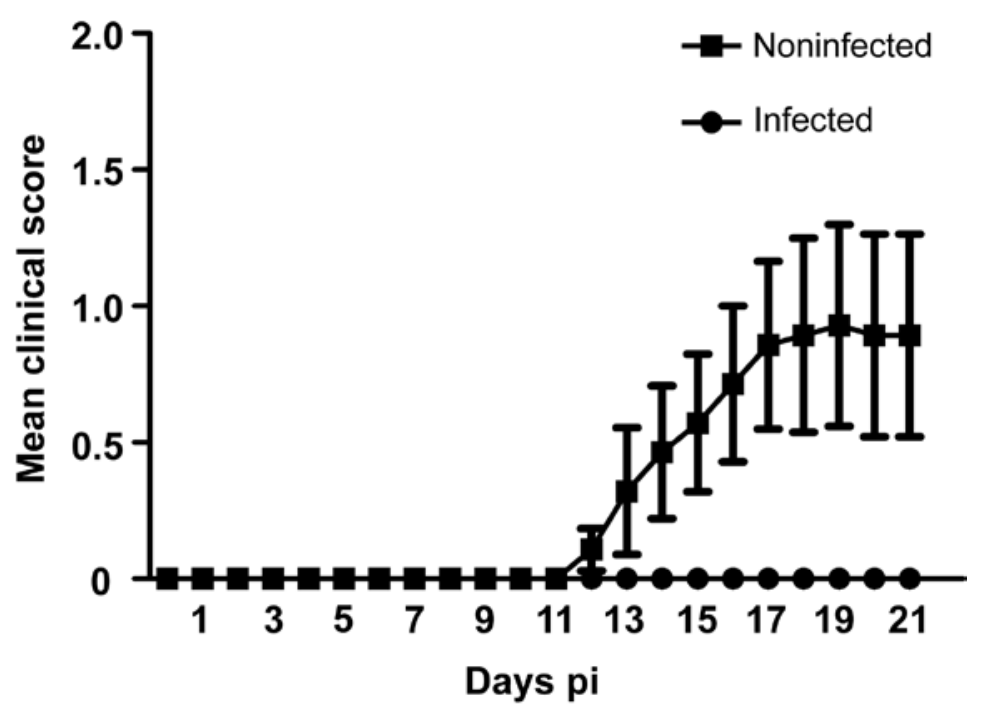

Fig 4.2.4: Clinical score: Infection with S. aureus on day 4 pi prevented disease onset in MOGEAE. Data presented as means \pm S.E.M. of the daily score.

Around day 13 after immunization, 6 of the 14 placebo-infected animals developed clinical symptoms while infection with $S$. aureus prevented the clinical manifestation of $E A E$ in all the infected animals. At the end of the experiment the mean cumulative score differed significantly between the group infected with $S$. aureus and the respective control group (no score vs $0.89 \pm 0.4 ; P=0.047$; Fig 4.2.4).

\subsection{4: Effect of $S$. aureus infection on neurodegeneration}

At day 8 of clinical disease or at day 21 after immunization in the absence of manifest disease, we determined the extent of demyelination of the optic nerves (ONs) by Luxol Fast Blue staining. Additionally, we performed immunohistochemistry for ED1 macrophages/microglia and $\mathrm{CD}^{+} \mathrm{T}$-lymphocytes to assess the extent of inflammatory infiltration. The chronic axonal damage was assessed by Bielschowsky's silver impregnation. For each staining, ON cross sections of three different levels were evaluated. 
A

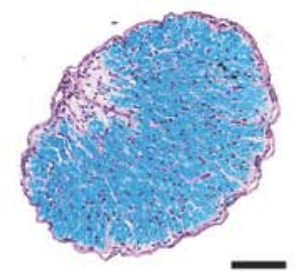

B

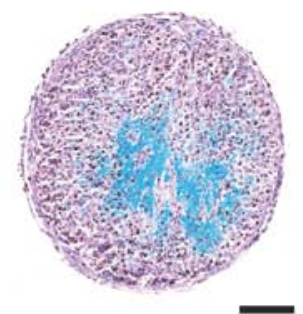

I

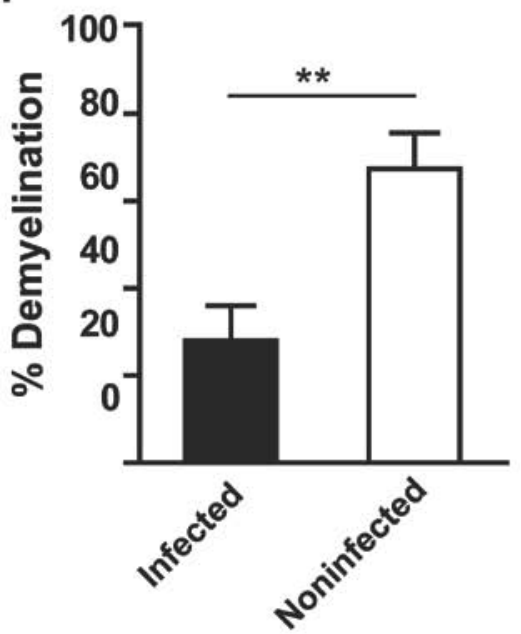

C

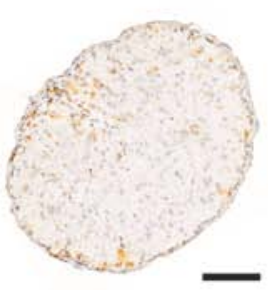

D

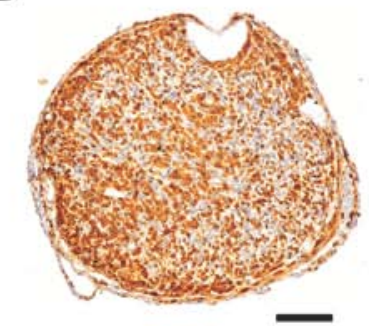

$\mathbf{J}$

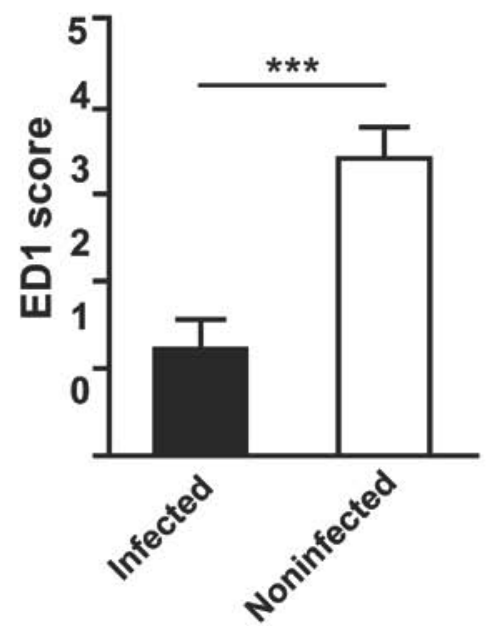

E

$\mathbf{F}$

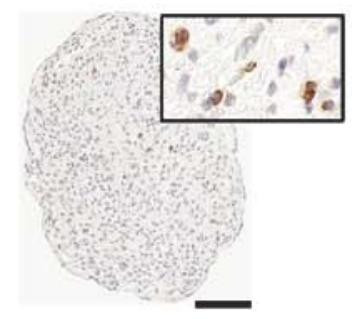

K

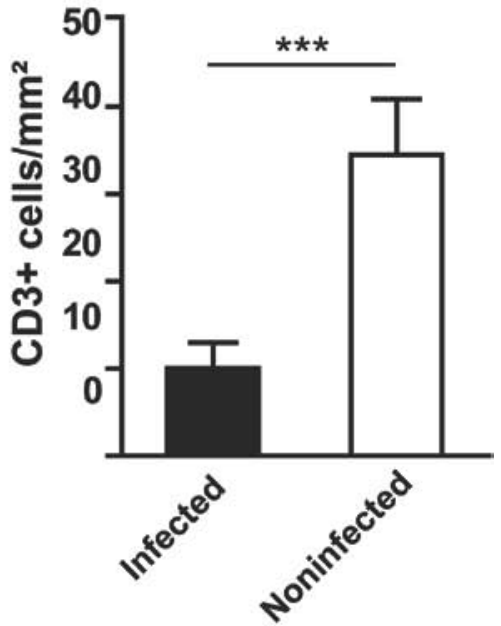

Fig 4.2.5: Optic nerve (ON) histopathology: $A$ and $B$ : Representative Luxol Fast Blue-stained cross sections of ONs of a wild-type $S$. aureus infected rat (A) showed only small areas of demyelination, with mainly intact myelin (blue) ; in contrast, a from a noninfected control animal (B) showed extensively demyelinated areas (purple). $\mathbf{C}$ and $\mathbf{D}$ : Representative examples of the substantially higher number of ED1 macrophages / activated microglia detected in the ON of a noninfected control animal (D), compared with an animal infected with wild-type S. aureus (C). E and F: representative images for CD3-positive T-Lymphocyte counts, which were noted higher in the optic nerve of the animals from the control group (F) whereas CD3-positive cells were hardly present in the optic nerve of the group infected with bacteria $(\mathbf{E}) . \mathbf{I}, \mathbf{J}$, and $\mathbf{K}$ represent the quantitative data for demyelination, ED1 score and CD3-positive cells respectively. Bar length = $100 \mu \mathrm{m}$. 
In the animals infected with the $S$. aureus wild-type strain we observed significantly less demyelination in ON cross sections (28.08 \pm 7.94\%; Fig 4.2.5 A, l; $n=16)$ compared to noninfected control animals (67.41 $\pm 8.17 \% ; P=0.0011$; Fig 4.2.5 $\mathrm{B}, \mathrm{l} ; \mathrm{n}=14)$. Analysis of inflammatory infiltrates with ED1 staining revealed a substantially lower infiltration by macrophages and activated microglia in the infected group (1.22 \pm 0.33 ; Fig 4.2.5 C, J; $\mathrm{n}=16)$ compared to the control group (3.41 $\pm 0.35 ; P<0.001$; Fig 4.2.5 D, J; $\mathrm{n}=14$ ). Quantification of $\mathrm{CD}^{+}$cells revealed that only the minority of infiltrating cells were $\mathrm{CD} 3^{+}$ T-lymphocytes. At day 21 after immunization, numbers of $\mathrm{CD}^{+}$cells were found to be significantly lower in the $S$. aureus infected group $\left(10.09 \pm 2.88 / \mathrm{mm}^{2}\right.$; Fig $4.2 .5 \mathrm{E}, \mathrm{K}$; $\mathrm{n}=16)$ in comparison to the noninfected control group $\left(34.46 \pm 6.39 / \mathrm{mm}^{2} ; P<0.001 ;\right.$ Fig 4.2.5 F, K; $n=14)$.
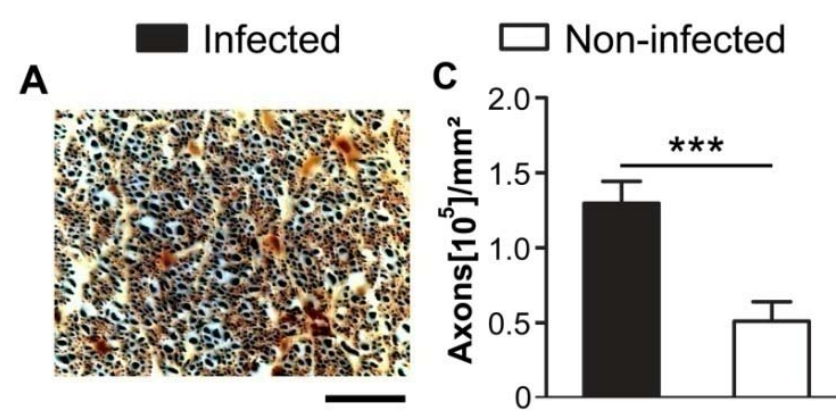

B

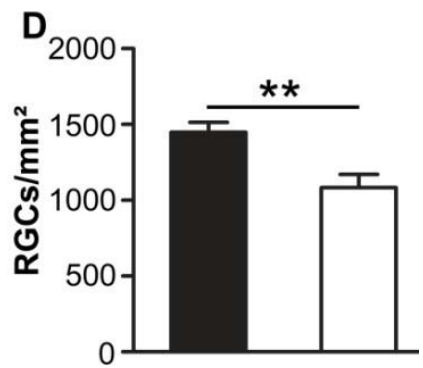

Fig 4.2.6: Axonal loss and RGCs counts: Representative Bielschowsky's silver impregnation of ON cross sections revealed a higher density of axons on EAE day 8 in wild-type infected animals (A) compared to noninfected control animals (B). C; shows the quantitative data for axonal loss between the group of animal infected with $S$. aureus and the noninfected group. Retrogradely labeled RGCs counting reveled a higher number of RGCs in the $S$. aureus infected group (D). Bar length $=20 \mu \mathrm{m}$.

Using Bielschowky's silver impregnation we observed a higher axonal density in $S$. aureus infected animals $\left(129700 \pm 14580 / \mathrm{mm}^{2}\right.$; Fig 4.2.6: $\left.A, C ; n=16\right)$ compared to 
noninfected control animals (51260 $\pm 12810 / \mathrm{mm}^{2} ; P<0.001$; Fig 4.2.6: $\left.\mathrm{B}, \mathrm{C} ; \mathrm{n}=14\right)$. To evaluate the impact of infection on neurodegeneration, numbers of surviving RGCs, the neurons that form the axons of the ON, were counted on day 8 of MOG-EAE. Previously, we have shown that in healthy $B N$ rats sham-immunized with complete Freund's adjuvant (CFA), $2730 \pm 145 \mathrm{RGCs} / \mathrm{mm}^{2}$ were detectable (mean $\pm \mathrm{SEM} ; \mathrm{n}=8$ ) (Meyer et al., 2001). In our present study, the mean RGC density in noninfected control animals at day 8 of MOG-EAE dropped to $1085 \pm 85.7 / \mathrm{mm}^{2}$ (Fig 4.2.6: $\mathrm{D}, \mathrm{n}=14$ ). In contrast, the RGCs density of infected animals was significantly higher (1449 \pm 64.60/mm², $P=0.002$; Fig 4.2.6: $\mathrm{D}, \mathrm{n}=20$ ).

The results from clinical studies indicated a protective effect of $S$. aureus infection on course of disease and at the same time $S$. aureus infection also improved neuropathological damaged induced by MOG-immunization by reducing antiinflammatory activity in the CNS. 


\section{3: Effect of Eap-deficient strain of S. aureus on EAE}

These results were surprising because, despite of a strong systemic inflammation induced by locally applied chronic $S$. aureus infection the animal did not show any clinical sign of EAE and showed reduction in number of inflammatory infiltrate in the optic nerve.

It is well known that $S$. aureus secrete various factors which could exert an antiinflammatory effect during the infection. An extracellular adhesion protein secreted by this bacterium has anti-inflammatory property which has been found to be present in $97.9 \%$ of clinical isolates (Hussian et al., 2001). Furthermore, anti-inflammatory effect of Eap secreted by $S$. aureus was demonstrated in chronic infections as well as in autoimmune disease models (Chavakis et al., 2002; Lee et al., 2002; Wang et al., 2010). Therefore, we hypothesize that this protein to be responsible for the protective effect of $S$. aureus infection in EAE. To prove our hypothesis a second set of experiments was performed by including a group of animals infected with $S$. aureus strain deficient for Eap (AH12), which was an isogenic mutant of wild-type Newmen strain of S. aureus (ATCC 25904).

\subsubsection{Infection with S. aureus strain deficient for Eap and systemic inflammation}

In this experiment leukocyte counts were significantly elevated two days after infection in the animals infected with a wild-type strain $(17236 \pm 742 / \mu \mathrm{l} ; \mathrm{n}=11 ; P<0.001)$ and in the animals infected with an Eap-deficient strain $(18575 \pm 585 / \mu \mathrm{l} ; \mathrm{n}=10 ; P<0.001)$ in contrast to the control group $(9940 \pm 527 / \mu \mathrm{l} ; n=5)$. Moreover, TCF plating revealed the presence and absence of bacteria in tissue cage fluid of both the infected and control group respectively. Although the exact determination of bacterial count was not possible due to the semi-solid consistency of TCF obtained from both the infected groups. However, we did not observe any significant difference between the leukocyte counts from both the infection groups at any time point during the whole course of the experiment. These results indicated that the level of infection in both the bacterial infected groups was similar and that should not be a region for any discrepancies in the results obtained from the group of animals infected with either wild-type S.aureus 
(ATCC 25904) or the animals infected with strain of the $S$. aureus deficient for Eap $(\mathrm{AH} 12)$.

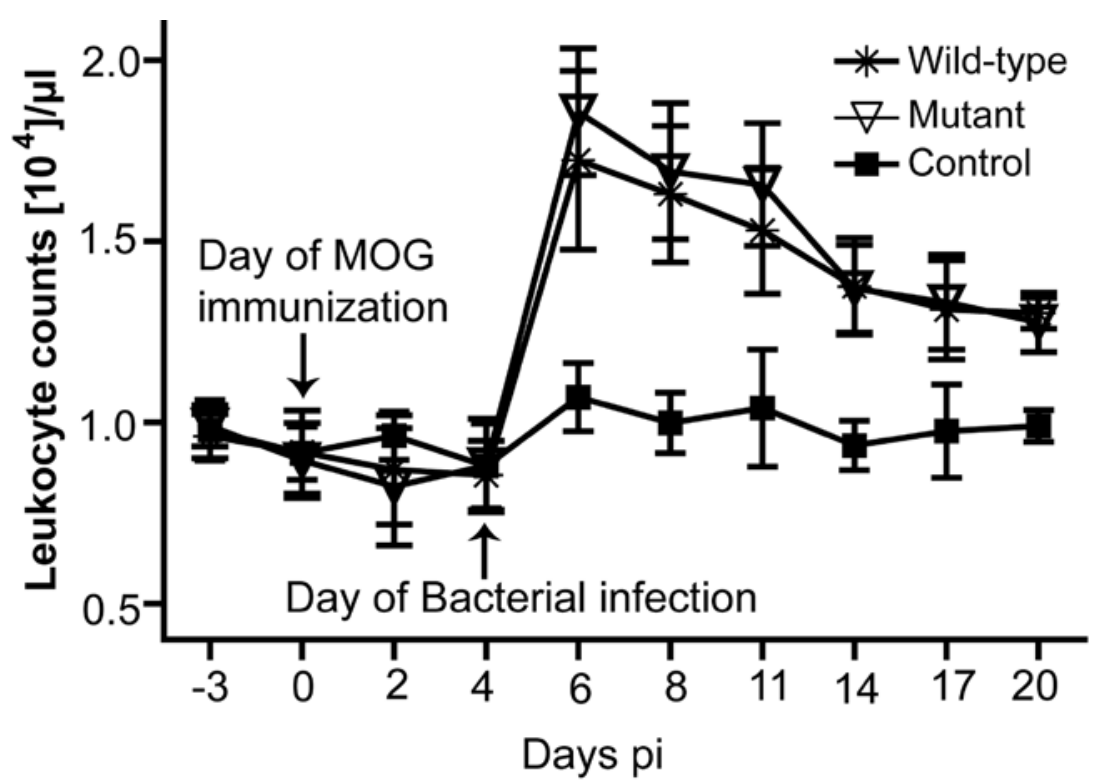

Fig 4.3.1: Blood leukocyte counts: - Leukocyte counts performed in peripheral blood of animals infected with, Eap-deficient bacterium (Mutant), S. aureus wild-type bacterium (Wildtype), and in saline injected myelin oligodendrocyte glycoprotein- (MOG) immunized animals (Control). The leukocyte counts were found to be significantly higher in both the infected group 2 days after the infection. Data are presented as means \pm SEM of all animals from each group.

\subsection{2: Infection with $S$. aureus and cytokines release in the systemic circulation}

In spite of Eap exhibit anti-inflammatory activity during infections and do not resemble the structural homology to superantigens (Haggar et. al., 2010), the binding of Eap to ICAM-1 on the surface of peripheral blood mononucleocytes triggers the release of proinflammatory cytokines like TNF- $\alpha$ and IL-6 (Scriba et.al., 2008). However, determinations of cytokines from in vivo is rather difficult, but in this experiment concentration of 12 cytokines were measured simultaneously by using the Bioplex 200system in the serum samples collected at the end of the experiment. These results indicated that animals infected with the wild-type strain of $S$. aureus showed a significantly higher level of IFN-gamma, IL-6 (Fig 4.3.2). The quantitative levels of the cytokines measured in the serum samples are indicated in table 4.3.1. 


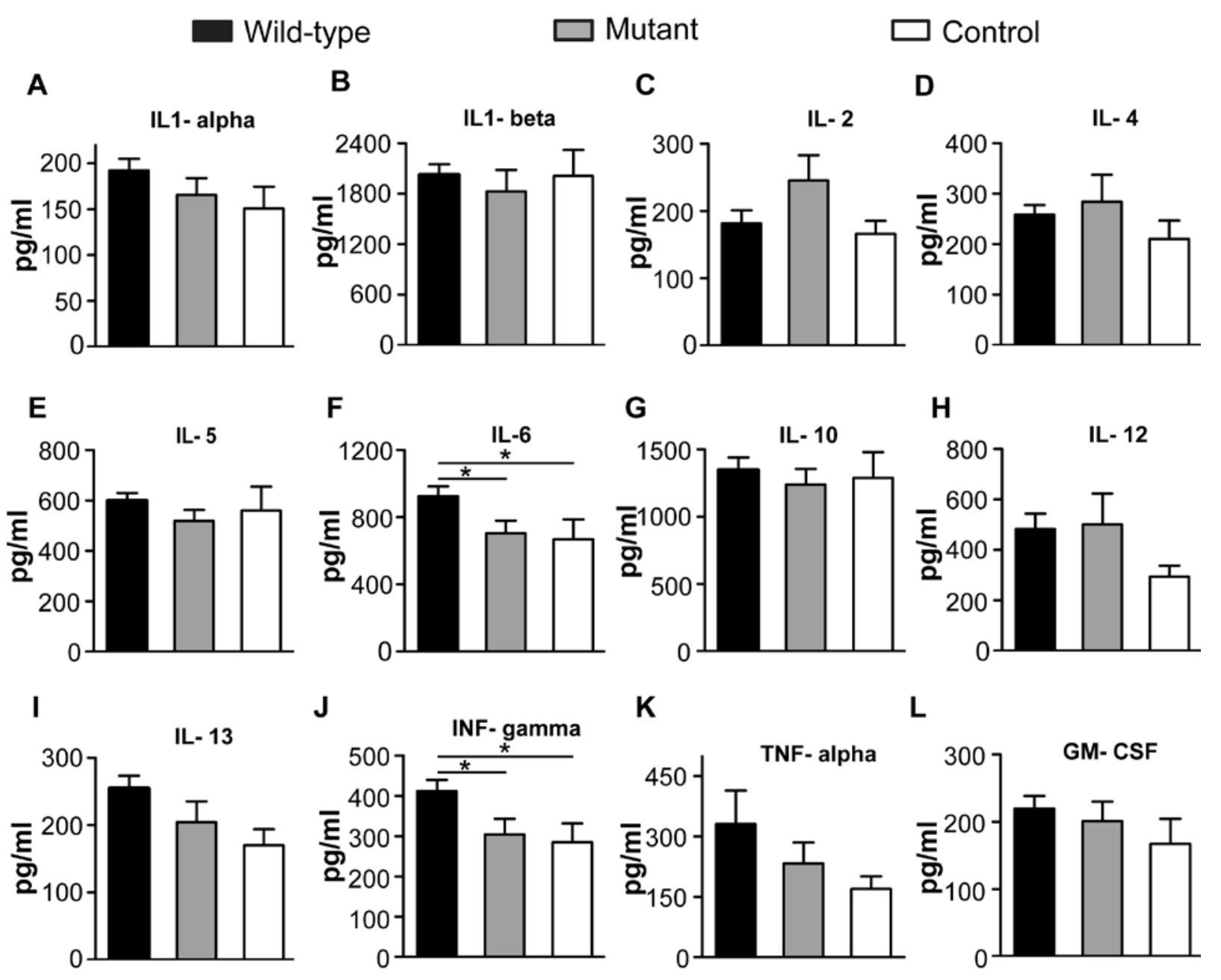

Fig 4.3.2: Luminex assays. Luminex detection assays indicated a significant increase of IL-6 (F) and IFN-gamma (J) and in the blood of animals infected with S. aureus wild-type strain.

More detailed, levels of IFN-gamma (Fig 4.3.2 J) was significantly increased in animals infected with the wild-type bacteria $(411.4 \pm 28.37 \mathrm{pg} / \mathrm{ml})$ compared to animals infected with the Eap-deficient strain $(303.9 \pm 39.08 \mathrm{pg} / \mathrm{ml} ; P=0.035)$ and compared to noninfected control animals (284.9 $\pm 46.49 \mathrm{pg} / \mathrm{ml} ; P=0.029)$. Serum levels of IL-6 (Fig 4.3.2 F) were also significantly elevated in the group infected with the wild-type $S$. aureus bacterium $(926.6 \pm 57.27 \mathrm{pg} / \mathrm{ml})$ compared to the group infected with the Eapdeficient bacterium $(704.4 \pm 75.48 \mathrm{pg} / \mathrm{ml} ; P=0.028)$ and the control group (668.7 \pm $119.1 \mathrm{pg} / \mathrm{ml} ; P=0.042$ ). A tendency towards higher serum levels of TNF- $\alpha$ (Fig 4.3.2 K) and IL-13, an anti-inflammatory cytokine (Fig 4.3.2 I) was observed in the animals 
infected with wild-type S. aureus bacteria compared to the group infected with Eapdeficient $S$. aureus and the noninfected control group.

\begin{tabular}{|l|c|c|c|}
\hline Cytokines & \multicolumn{1}{c}{$\begin{array}{c}\text { Wild-type group } \\
\mathbf{( p g / m )}\end{array}$} & $\begin{array}{c}\text { Eap-deficient group } \\
\mathbf{( p g / m l )}\end{array}$ & $\begin{array}{c}\text { Control } \\
\mathbf{( p g} / \mathbf{m l})\end{array}$ \\
\hline IL1 -alpha & $192.3 \pm 12.70$ & $165.5 \pm 18.54$ & $150.7 \pm 23.82$ \\
\hline IL-1 beta & $2025 \pm 125.9$ & $1827 \pm 256.3$ & $2012 \pm 308.1$ \\
\hline IL-2 & $181.7 \pm 19.44$ & $245.7 \pm 37.52$ & $166.1 \pm 19.57$ \\
\hline IL-4 & $258.6 \pm 18.98$ & $284.1 \pm 53.28$ & $210.6 \pm 36.45$ \\
\hline IL-5 & $599.9 \pm 29.66$ & $519.9 \pm 42.49$ & $560.6 \pm 94.28$ \\
\hline IL-6 & $926.6 \pm 57.27$ & $704.4 \pm 75.48$ & $668.7 \pm 119.1$ \\
\hline IL-10 & $1348 \pm 92.67$ & $1239 \pm 117.0$ & $1290 \pm 191.8$ \\
\hline IL-12 & $483.3 \pm 60.40$ & $500.9 \pm 122.7$ & $293.4 \pm 43.98$ \\
\hline IL-13 & $254.8 \pm 18.78$ & $204.4 \pm 31.00$ & $170.2 \pm 23.76$ \\
\hline TNF- alpha & $330.7 \pm 82.96$ & $233.2 \pm 51.44$ & $169.9 \pm 30.89$ \\
\hline IFN- gamma & $411.4 \pm 28.37$ & $303.9 \pm 39.08$ & $284.9 \pm 46.49$ \\
\hline GM- CSF & $219.4 \pm 19.10$ & $201.2 \pm 28.80$ & $167.1 \pm 37.49$ \\
\hline
\end{tabular}

Table 4.3.1: Quantitative data for cytokine analysis: Table represents the quantity of cytokine concentration in the serum sample collected at the end of the experiment.

Although the results were not significant but the group of animals infected with Eapdeficient bacteria also showed a tendency towards higher of proinflammatory cytokines such as IL2 and IL12. Therefore these data showed that the pro- and anti-inflammatory 
activity in the periphery is ongoing together, indicating that the variation in the cytokines profile could have influenced on disease severity.

\subsection{3: S. aureus infection and its impact on clinical manifestation of the disease}

Concerning the clinical manifestation of the disease, no clinical signs of EAE were observed in the group infected with S. aureus wild-type as well as in the group infected with the Eap-deficient strain. In contrast, noninfected control animals were severely affected with an EAE score of $2.4 \pm 0.36(n=5 ; P<0.001)$ at the end of the experiment. Although no clinical signs of EAE were observed in the group infected with $S$. aureus or in the group infected with the Eap-deficient strain of $S$. aureus, animals of the group infected with Eap-deficient $S$. aureus displayed higher level of histopathological abnormalities (see below).

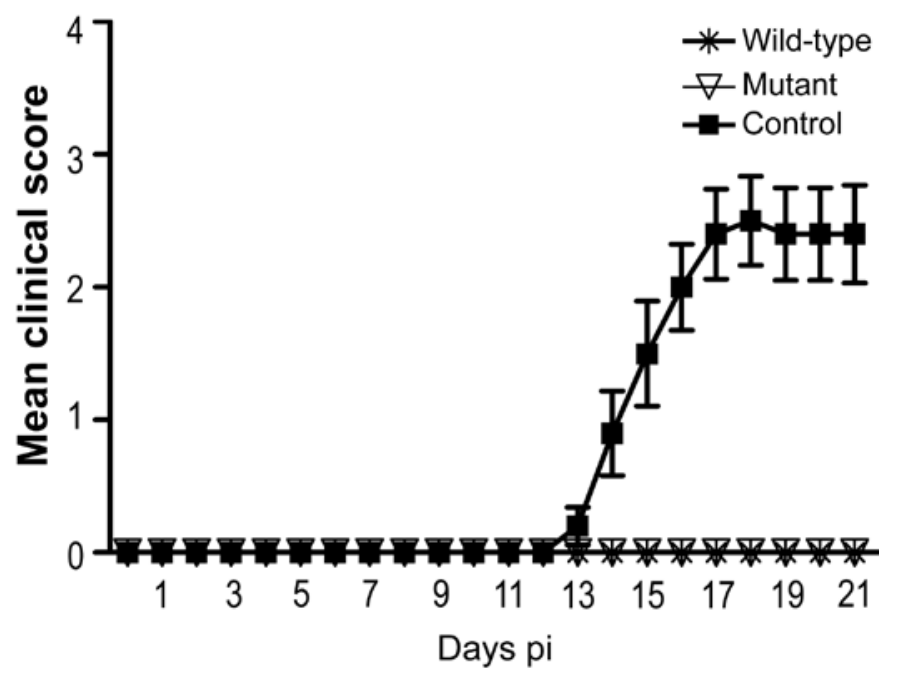

Fig 4.3.3: Clinical manifestation of disease: Clinical manifestation of disease was abolished completely in animals infected with $S$. aureus bacteria.

\subsection{4: Neuropathological damage and RGCs survival after Infection with Eap- deficient strain of $S$. aureus}

Histopathilogical analysis performed on the optic nerve s revealed that the protective effect of wild-type $S$. aureus infection on optic neuritis is reduced in the group infected with respective Eap-deficient strain. 
In more detailed, we observed significantly less demyelination (Fig 4.3.4 A) in wild-type $S$. aureus infected animals (22.77 $\pm 8.46 \%$; $n=11)$ in comparison to both the animals groups infected either with $S$. aureus deficient for Eap $(53.53 \pm 10.56 \% ; P=$ $0.027 ; n=10)$ or the control animals $(100 \pm 0 \% ; P<0.001 ; n=4)$. Accordingly, the extent of inflammation accessed by ED1 staining (Fig 4.3.4 B) was significantly lower in the group infected with wild-type $S$. aureus bacteria $(1.24 \pm 0.39 ; n=11)$ in comparison to the group infected with Eap-deficient bacteria $(2.73 \pm 0.40 ; P=0.012 ; n=10)$ and the noninfected control group (4.66 $\pm 0.15 ; P<0.001 ; \mathrm{n}=4)$.
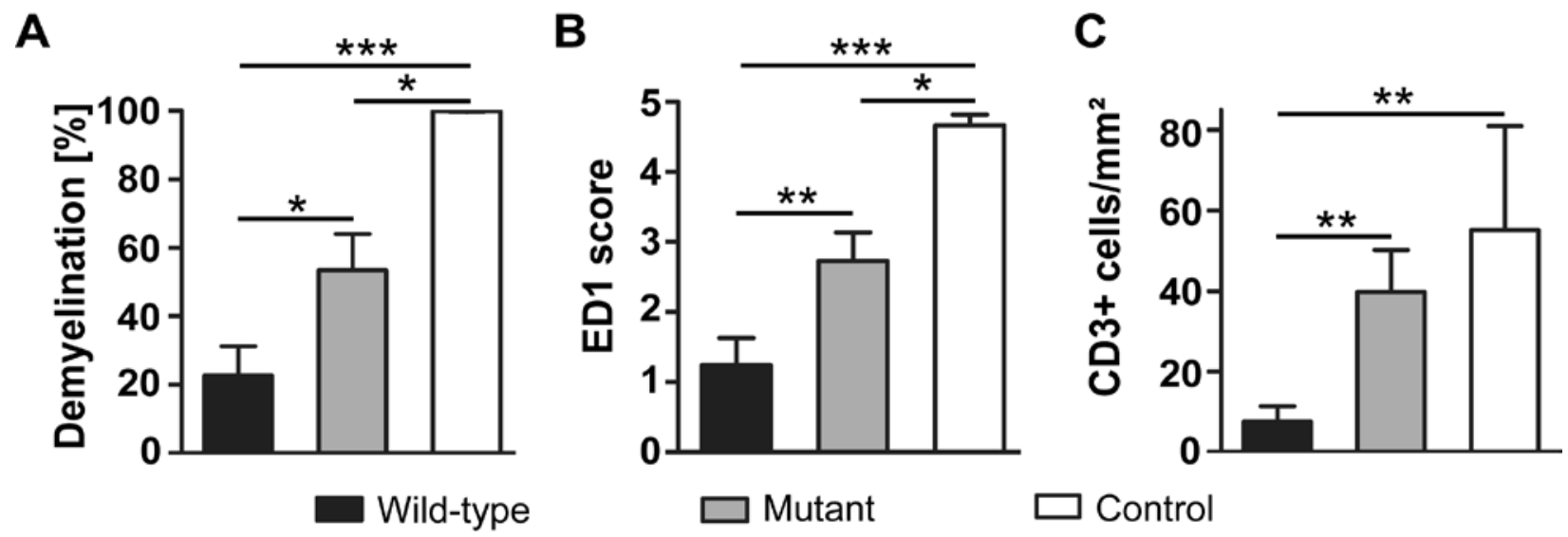

Fig 4.3.4: Optic nerve (ON) histopathology after infection with wild type $S$. aureus along with the strain deficient for Eap: The positive effect of $S$. aureus on optic neuritis was reverted in the Eap-deficient strain. A, B and $\mathbf{C}$ represents the quantitative data for extent of demyelination, $\mathrm{ED} 1$ score and $\mathrm{CD}^{+}$cells respectively.

$\mathrm{CD}^{+}{ }^{+}$T-Lymphocyte counts revealed significantly lower numbers of $\mathrm{CD}^{+}$cells (Fig 4.3.4 C) in animals infected with wild-type $S$. aureus $\left(7.6 \pm 3.75 / \mathrm{mm}^{2} ; \mathrm{n}=11\right)$ in comparison to the animals infected with Eap-deficient bacteria (39.81 $\pm 10.37 / \mathrm{mm}^{2} ; P=0.004 ; \mathrm{n}=10$ ) and the control group $\left(55.2 \pm 25.8 / \mathrm{mm}^{2} ; P=0.007 ; \mathrm{n}=4\right)$. No significant difference was observed in $\mathrm{CD}^{+}$cell counts between the animals infected with Eap-deficient $S$. aureus bacteria and the noninfected control group $(P=0.5)$.

Bielschowsky's silver impregnation was performed to assess the axonal pathology of the optic nerve (Fig 4.3.5 A) and revealed that the animals infected with wild-type bacteria showed a higher number of axonal counts $\left(173500 \pm 18220 / \mathrm{mm}^{2}\right.$; 
$\mathrm{n}=11$ ) in comparison to the group infected with Eap-deficient bacteria (73953 \pm $\left.14500 / \mathrm{mm}^{2} ; P<0.001 ; \mathrm{n}=10\right)$ and noninfected control animals $\left(16670 \pm 1938 / \mathrm{mm}^{2} ; P<\right.$ $0.001 ; n=4)$.
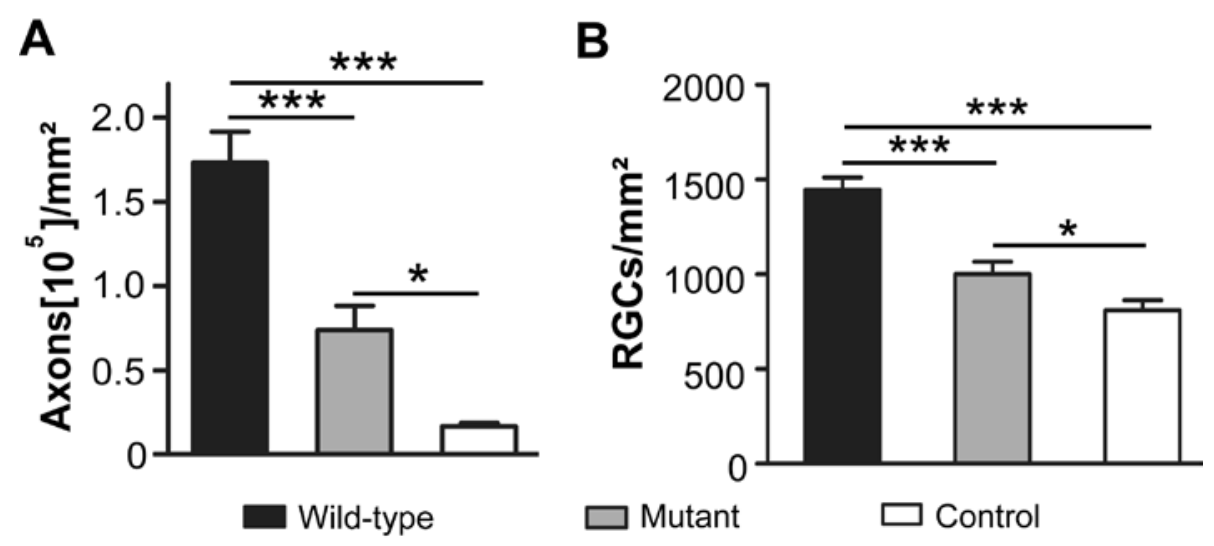

Fig 4.3.5: Axonal loss and RGCs count after infection with wild type $S$. aureus along with the strain deficient for Eap (mutant): Bielschowsky's silver impregnation (A) of ON cross sections revealed a significantly higher density of axons on EAE day 8 in wild-type infected animals. Retrogradely labeled RGCs counting (B) showed a higher number of RGCs in the group of animals infected with wild-type $S$. aureus bacterium.

The RGCs counts were performed on retinal flat mount which revealed a higher RGC density in the animal group infected with the wild-type strain $\left(1447 \pm 66 / \mathrm{mm}^{2}\right)$ compared to animals infected with the Eap-deficient strain $\left(1001 \pm 65 / \mathrm{mm}^{2} ; P<0.001\right.$; Fig 4.3.5) and the noninfected control group $\left(810 \pm 52 / \mathrm{mm}^{2}, P<0.001\right.$; Fig 4.3.5).

Although the infection with Eap-deficient $S$. aureus bacterium has a protective effect on the clinical course of disease but these results also indicated that the infection with wild-type bacterium is more protective in terms of optic nerve injury and RGCs counts compared to the animals infected with bacterium deficient for Eap or the control animals. 


\section{DISCUSSION}

There is an extensive clinical literature describing the effect of infections on clinical course of MS, mostly on exacerbation of the disease course, but in some instances reducing relapses especially in case of parasitic infection (Correale and Farez, 2011; Correale and Farez, 2013; Fleming and Cook, 2006). In the present work we investigated the extent to which an acute infection with $E$. coli and chronic infection with $S$. aureus influences the course and severity of MOG induced EAE in female Brown Norway rats.

\section{1: Infection and MS}

There is a growing body of evidence that the progression of MS is influenced by systemic infection caused by bacteria or viruses. Moreover, components associated with certain bacteria and viruses have been shown to be a potential modifier in the progression of autoimmune diseases (Hamada et al., 1989; Nogai et al., 2005; Soos et al., 1995; Xie et al., 2006). However, only few studies have investigated the direct impact of infection with viable bacteria in the animal models of MS, which reflect more likely a clinical situation. The results of MRI on the influence of peripheral infection and the number of contrast-enhancing MS lesions as a marker of disease activity are inconsistent (Edwards et al., 1998; Kalkers et al., 2002). Moreover, reliable markers for neurodegeneration in MS patients do not exist. For this reason, much of the knowledge about the interaction of systemic infection and autoimmunity in MS is derived from studies in EAE models. Previous studies in EAE models mainly focused on analyzing the clinical course of the disease. Furthermore, in most experiments solely bacterial compounds were used to induce systemic inflammation (e.g. LPS, enterotoxins) (Moreno et al., 2011; Schiffenbauer et al., 1993; Soos et al., 1995) instead of whole viable bacteria, therefore these studies showed the limited value concerning the reflection of the clinical situation in MS-patients. 


\subsection{1: Acute infection with E. coli and EAE}

Clinical as well as experimental data indicated that the preclinical phase prior to the disease manifestation to be a period with the highest susceptibility to infections (Herrmann et al., 2006). However, during the disease course of MS, infections with common bacteria are also observed, which could have the potential to modulate the disease severity. Therefore, to elucidate the impact of infections in our study, two different infection time points were selected. In the first group, animals were infected at day 7 post immunization corresponding to the preclinical phase of the disease. Furthermore, to reflect the clinical situation a second group of animals was infected on day of clinical onset of disease. To increase the survival of animals after the infection, they were treated with ceftriaxone, a beta lactam antibiotic used for the cure of bacterial infection. The effect of beta lactam antibiotic in an animal model of MS remains controversial. In the study of Nico Melzer and coworkers, it was shown that the ceftriaxone treatment ameliorated the clinical course of MOG-induced EAE in mice by modulating cellular antigen-presentation and impairment of antigen-specific T-cell migration into the CNS (Melzer et al., 2008). In contrast to this finding Felix Mor and colleagues showed that adoptive transfer of BP10 cell line incubated with ceftriaxone shown more severe EAE symptoms (Mor and Cohen, 2013). Moreover, they also showed that cefuroxime, another beta lactam antibiotic enhanced adjuvant-induced arthritis and experimental autoimmune encephalomyelitis (EAE) in both active and passive forms. However, to rule out the side effect of ceftriaxone in our study all the animals from infected and noninfected group was treated with ceftriaxone during the experimental phase of disease.

Viral and bacterial infections can aggravate the disease symptoms in MS patients (Buljevac et al., 2002). Moreover, in the previous experimental studies in EAE it has been shown that the, bacteria like Chlamydia pneumoniae and Streptococcus pneumoniae could exacerbate the disease symptoms (Du et al., 2002; Herrmann et al., 2006). Here, in our study, in spite of a systemic infection with $E$. coli in the preclinical phase of disease induced weight loss in EAE animals considering as a sign of general sickness behavior, it has no influence neither on the time point of onset of clinical EAE 
symptoms nor on the severity of the disease. Moreover, to reflect the clinical situation, we infected the animals on the clinical onset of EAE. Despite the presence of bacteria in the systemic circulation of $E$. coli infected animals next days after clinical onset of disease it did not influence the disease severity. Further, histopathological analysis performed on the optic nerves of animals did not reveal any significant increase in inflammatory infiltrates in the animals infected with $E$. coli either on day 7 post immunization or on day 1 EAE compared the respective control. Further, in this study, we did not observe any significant difference in other histopathological correlates like demyelination and axonal counts on the optic nerves in the group infected with $E$. coli compared to the control.

Results obtained from the acute infection with $E$. coli in this animal model, did not reflect the previous findings, those which were performed with the bacterial components and induce a robust, but short lasting systemic inflammation. It has been shown that $E$. coli infection increases the permeability of the BBB during sepsis, but unable to cross the BBB (Tsao et al., 2001). Moreover, it has also been suggested that infection of CNS is necessary for worsening of EAE (Du et al., 2002). These experimental data could possibly explain the discrepancies in our finding compared to the previous experimental studies. However, other possible reasons for such discrepancies in our result might be due to balance between Th1/Th2 cytokine profile, molecular mimicry and the role of Toll like receptors (TLRs). These finding also suggested that the kind of micro-organism also has influence on exacerbation of EAE relapse and their histopathological changes.

\subsection{2: Chronic infection model}

Foreign body infections are characterized by the presence of adherent bacteria on the surface of the synthetic prosthetic materials for a very long time and these bacteria supposed to escape host dependent phagocytic mechanisms. S. aureus is a common pathogen in both hospital- and community-associated infections (Grady and Cullen, 2003) and cause serious complications in prostheses by formation of biofilm and developing tolerance towards an antibiotic treatment (Murillo et al., 2006). S. aureus contains or secretes around 200 products, which may interfere with immune- 
inflammatory reaction and can confer virulence of the host. Moreover, the involvement of some of the bacterial components, isolated from $S$. aureus, in the modulation of EAE was demonstrated in previous experimental studies (Schiffenbauer et al., 1993; Soos et al., 1995; Xie et al., 2006). Therefore, these observations indicated, S. aureus to be the ideal microorganism to study the effect of chronic infection in vivo. Lucet and co-workers established an experimental model of chronic foreign body infection by inoculating $10^{5}$ $10^{6} \mathrm{CFU}$ of $\mathrm{S}$. aureus in the implanted tissue cages containing two PMMA cover slips in Wister rat (Lucet et al., 1990).

However, in our study tissue cages implant with PMMA cover slips was unable to induce the chronic infection even with a high inoculum dosage of $10^{9} \mathrm{CFU}$ of $\mathrm{S}$. aureus in $\mathrm{BN}$ rat. To overcome this problem semi-solid agar, an excellent medium for the growth of bacteria, was injected directly into the implanted tissue cages of the animals prior to infection. That further leads to a persistent chronic infection in this animal model. The advantage of this chronic infection model is that, it induces mild infection and even without antibiotic treatment, it does not lead to death of the animal. A major advantage of agar injection into the implant was that, it reduces the inoculum dosage of $S$. aureus to induce the chronic infection, which allow us to stabilize the implant in the body of the animals for longer time duration.

\subsection{3: Chronic infection with $S$. aureus, induces systemic inflammation}

Infection with bacteria induces the sign of sickness behavior, which reflects in the form of weight loss (Perry, 2010). Interestingly, animals infected with S. aureus did not show any weight loss after the infection. Moreover, during the course of the experiment we did not observe the presence of bacteria in the peripheral blood of animals, when plated on the blood agar plate. However, at the end of the experiments, TCF obtained from the animals infected with $S$. aureus showed a high number of bacterial counts (S. aureus) on the blood agar plate, whereas no bacterial growth was observed in TCF obtained from noninfected control animals. The data obtained from TCF, obtained at the end of the experiments, plated on the blood agar plate confirmed the persistent chronic infection in the tissue cage of the animals. Moreover, leukocyte counts in the peripheral 
blood from the $S$. aureus infected animals showed a significant increase in the blood leukocyte counts, 2 days after the local application of the bacteria in the tissue cage. Further, flow-cytometric analysis of the peripheral blood circulation also showed a significant increase in the population of B- and T-cell count in the animals infected with S. aureus. Furthermore, the flow cytometric analysis also indicated a more pronounced increase in the T-cell subpopulation 2 days after the infection, compared to the control. These results confirm that a chronic infection with $S$. aureus leads to a very strong systemic inflammatory response in the peripheral blood circulation of the animals.

It has been shown that after the infection, Infectious agents can expand the pool of host's immune cells, which also includes auto-reactive T-cells in EAE and thus can contribute to the disease progression (Du et al., 2002; Nogai et al., 2005; Visser et al., 2005). Thus we hypothesized that due to increase in leukocyte counts and a substantial increase of B-, T- and subpopulation of T-cell in peripheral blood after the infection, it will lead to more severe damage to the brain tissue and possibly could exacerbate the symptoms of EAE. Unexpectedly, the infection with $S$. aureus prevented the development of clinical EAE. Moreover, it significantly decreased the extent of inflammatory infiltrates and demyelination of the optic nerve. However, these results can be explained on the basis of hygiene hypothesis which states that infections protect rather than induce/accelerate autoimmune diseases like MS (Bach, 2002). The hygiene hypothesis proved to be protective in several clinical and experimental studies (Correale and Farez, 2011). Moreover, hygiene hypothesis also state that due to hyper-activation of auto-reactive cells with further activation leads to cell death and may reduce the number of aggressive cell number. Moreover, the competitive mechanisms between the infectious agent and the body own immune cells for the antigen binding over MHC molecules and antigen processing by phagocytic cells could also play an important role in this protective function. In the previous finding it has been shown that the induction of superantigens produced by different infectious agents can exacerbate the EAE (Schiffenbauer et al., 1993). However, these findings could have a different prospective as well. It is well known that after the antigen presentation on MHC grove it leads to the activation of specific T-cell population bearing particular T-cell-receptor in variable regions of the beta chain. However, the presence of superantigen induces polyclonal T- 
cell activation (Fraser and Proft, 2008). Hence, in my opinion of superantigen might cause a reduction of activated T-cell number expressing a particular TCR by modulation in the variable region of beta chain (Correale and Farez, 2011).

\subsection{4: Chronic infection with S. aureus and cytokine release}

It is well known fact that infection induces cytokine release in the periphery, which is crucial for fighting against infections. However, sometimes they can become dysregulated during inflammation, trauma, and sepsis (Dinarello, 2000). In multiple sclerosis how the regulations of different cytokine takes place are not clearly known. Experimental studies indicated that in EAE, the release of pro-inflammatory cytokines worsen the disease, whereas anti-inflammatory cytokines reduce inflammation and promote healing. Moreover, it has been shown that the pro-inflammatory cytokines like IL-1, IL-6, IL-12, IL-17, IL-23, TNF- $\alpha$, and IFN-gamma seems to increase the severity of the disease in EAE (Komiyama et al., 2006; Langrish et al., 2005; Samoilova et al., 1998). In contrast to this several experimental studies indicated the protective role of IFN-gamma and TNF-a in EAE (Furlan et al., 2001; Willenborg et al., 1996; Zhang et al., 2012).

In spite of anti-inflammatory activity of Eap during infection, in our study, we detected a significant increase in the level of the IL-6 and IFN-gamma levels at the end of the experiment in the group infected with $S$. aureus wild-type strains. However, such a pro-inflammatory cytokine pattern was not detected in animals infected with the Eapdeficient strain. Our findings correspond well to in vitro data showing increased secretion of IL- 6 and TNF- $\alpha$ in murine cells and in human blood samples after stimulation with Eap (Edwards et al., 2012; Scriba et al., 2008) and indicate proinflammatory properties of this protein in the systemic circulation. However, Scriba and coworkers also showed that this pro-inflammatory cytokine release is taking place through monocytes and macrophages but not by T-cell populations. Intriguingly, in spite

of ongoing inflammation in the periphery the infection with $S$. aureus prevented the onset of clinical EAE symptoms and decreased the severity of autoimmune optic neuritis. According to our hypothesis secretion of Eap blocks the migration of 
inflammatory cells into the CNS, which in turns protects the integrity of the BBB. However, less infiltration of T- and B-cells in the animals infected with $S$. aureus restrict the innate release of pro-inflammatory cytokines from these cells into the CNS. When the BBB is intact, the majority of the blood borne cytokines enters into the CNS by the saturable transport system (Banks, 2005; Banks et al., 1991), which facilitates the selective movement these cytokines across the BBB. However, during EAE the alteration in the permeability of the BBB allows an unrestricted movement of these cytokines into the CNS, which probably not the case in the animals infected with $S$. aureus. This could be a possible reason why ongoing inflammation in the periphery has no deleterious effect on the course of this disease.

\subsection{5: The role of adhesion molecules in transendothelial migration}

The entry of leukocytes into the CNS is considered as an early event in MS. That includes the involvement of cytokines, chemokines and adhesion molecules expressed by both the activated endothelium and the immune cells, which further leads to the breakdown of the BBB and inflammation of the CNS. The relevance of VLA-4 - VCAM-1 for migration of lymphocytes across the BBB during EAE has been shown in several experimental studies (Archelos et al., 1993; Gordon et al., 1995; Yednock et al., 1992). Moreover, several genetics studies also indicated the relevance of adhesion molecules in the susceptibility of the disease (Damotte et al., 2014). The co-ordinated sequential events of inflammatory cells migration into the CNS, especially, involve the VLA-4 VCAM-1 and the LFA-1 - ICAM-1 adhesion systems (Engelhardt and Wolburg, 2004; Laschinger and Engelhardt, 2000; Lee and Imhof, 2008; Wittchen, 2009). However, there is increasing evidence that both systems may have a distinct contribution in the recruitment of myelin activated T-cells. Experimental studies also indicated the organ specific expression of such molecules on their endothelium during autoimmune inflammation. Moreover, the subtype of T-cells that are involved in the pathology may vary in patients (Bitsch and Bruck, 2002; Metz et al., 2014). It has been shown, that Th1- and Th17-cells filtration in the spinal cord is dependent on a VCAM-VLA-4 interaction (Rothhammer et al., 2011). In contrast to this finding Bharadwaj and coworkers showed that the vascular endothelial migration of Th1- and Th17-cells was 
mediated by ICAM instead of VCAM expression (Bharadwaj et al., 2013). Hence it can be speculated from a previous finding that different subtypes of activated T-cells will be recruited on various adhesion molecules and crosses the BBB.

Humanized monoclonal antibody natalizumab is in therapeutic use for MS. In a clinical study it was observed that natalizumab treatment reduced the MRI activity by $92 \%$, which showed the highest efficacy for any drugs available for treatment of MS (Miller et al., 2007; Polman et al., 2006). Natalizumab treatment targets the a4-integrin molecule, a component of the VLA-4 present of lymphocytes thus prevents the interaction with VCAM found on endothelial surfaces and subsequently blocks the migration of the lymphocytes to the CNS, which resulted in the reduction of the inflammation in the CNS and disease severity of the disease. However, some patients do not respond to the natalizumab treatment, especially in patients with progressive forms of MS. This clearly indicates the heterogeneity of mechanism involve in the disease pathogenesis of MS. Another concerning factor for natalizumab treatment is the incidence of PML (2.1/1000 patients) which increases with the length of treatment (Bloomgren et al., 2012; Clifford et al., 2010). Moreover, the patient becomes more susceptible if they were treated with an immunosuppressant before receiving natalizumab (Kappos et al., 2011). In contrast to the VLA-4 - VCAM-1 interaction several studies indicated the role of ICAM in pathogenesis of MS (Bullard et al., 2007; Damotte et al., 2014; Steiner et al., 2010). Furthermore, the expression of ICAM-1 has been shown to be up-regulated in MS. Eventually there is no current therapy targeting the LFA-1 - ICAM-1 adhesion pathway.

Staphylococcal enterotoxins (SES) are one member of a unique group of molecules called super-antigens which could induce a very strong immune response in the animals. Moreover, it has been also shown that super antigens may trigger EAE by stimulation of autoreactive T-cells from the variable region of beta chain (Schiffenbauer et al., 1993; Soos et al., 1995). In contrast to this, S. aureus also secrete several antiinflammatory and anti-adhesive factors that may interfere with every step of inflammatory cells recruitment of the host (Chavakis et al., 2007) and help them for an efficient colonization within the host (Chavakis et al., 2005; Foster and Hook, 1998). 
S. aureus secrete a group of anti-adhesive protein consist of 5 different structurally unrelated secreted adhesion molecule named as "secretable expanded repertoire adhesive molecules" (SERAM), and one of them is Eap (Chavakis et al., 2007). Eap, also called as Map, "MHC class II analogous protein", which has been shown to be present in $97 \%$ of clinical isolates (Harraghy et al., 2003). The Eap not only help bacteria to get adhere but also interferes with host defense mechanisms. It has been shown that Eap specifically interacts with endothelial ICAM-1 and inhibit the recruitment of neutrophil at the site of infection. Furthermore, Eap also interfere with T-cell migration by blocking the interaction between LFA-1 and ICAM-1 (Athanasopoulos et al., 2006; Chavakis et al., 2002; Haggar et al., 2004). Moreover, an anti-inflammatory effect of Eap in autoimmune diseases has been found in EAE and an animal model of psoriasis (Wang et al., 2010; Xie et al., 2006).

Despite of a significant increase in T-cells count in the animals infected with $S$. aureus it showed a significantly less infiltration of T-cells on the optic nerve of the animals. These findings strongly suggested the involvement of a factor which, probably involved in the trans-migration of myelin activated T-cells across the BBB. Based on these observations, we hypothesized that the anti-inflammatory effects of $S$. aureus infection in MOG-EAE are mediated through the secretion of Eap. We infected the animals with the $S$. aureus strain deficient for Eap. In our study, the infection with the Eap-deficient $S$. aureus strain led to increased inflammation, demyelination and axonal damage in the optic nerve compared to the animals infected with the wild-type $S$. aureus strain. However, histopathological analysis of the optic nerve revealed that the antiinflammatory property of $S$. aureus infection was not completely abolished by lacking Eap. Moreover, the infection with the Eap-deficient strain prevented the clinical manifestation of the disease, indicating that additional mechanisms are involved in the anti-inflammatory effects of $S$. aureus. Given the importance of ICAM-1 antagonism in different autoimmune diseases (Kaneider et al., 2006), Eap is considered to be the most potent factor for inhibition of T-cell migration into the CNS. This hypothesis is supported by our histopahological evaluations revealing a substantial decrease of lymphocyte count in inflammatory infiltrates of the optic nerve in wild-type S. aureus infected animals. 


\section{2: Infection and neurodegeneration}

Epidemiological data implicated both genetic and environmental factors in the etiology of MS. The association of systemic infection and risk of MS relapse has been proposed on the basis of epidemiological data (Handel et al., 2010) from several clinical studies (Correale et al., 2006; Sibley et al., 1985). However, whether the infection leads to the increased neurodegeneration, a major hallmark of disability in MS patients is not fully understood.

Microglials are the innate immune cells of the brain and plays a very important role in the maintenance of the microenvironment of the CNS. In the presence of external stimulus these cells can become activated which is crucial for host defense mechanism. However, over-activation of microglia may cause neurotoxicity (McGeer et al., 2005; Polazzi and Contestabile, 2002). Peripheral inflammation, which leads to the activation of Microglial cells is a common phenomenon of several neurodegenerative diseases like Parkinson's disease, Alzheimer's disease, Multiple sclerosis and Amyotrophic Lateral Sclerosis (Nguyen et al., 2002). It is now well established, that in chronic neurodegenerative diseases of the CNS, such as Parkinson and Alzheimer diseases the resident microglia are primed by prior pathology to respond more vigorously to inflammatory stimulation. In this context, the signals from systemic infection or inflammation lead to a switch of microglia/macrophages towards a pro-inflammatory phenotype (Cunningham, 2013; Perry et al., 2007) and this switch contributes to progression of neurodegeneration (Ferrari et al., 2006; Nguyen et al., 2004).

The majority of studies on studying the interaction of systemic infection and autoimmunity have been performed on EAE animals using a single challenge with lipopolysaccharide (LPS), which is present on the outer membrane of Gram-negative bacteria. Systemic insult with LPS leads to a switch in the macrophage/microglia phenotype in the rat EAE model. This switch was accompanied by prolonged proinflammatory response by releasing inducible nitric oxide synthase (iNOS) and expression of pro-inflammatory cytokines, which in turns lead to the more pronounced neuronal damage (Moreno et al., 2011). But whether these bacterial components (LPS, enterotoxins, or teichoic acid, etc.), which are commonly used to mimic the situation of 
bacterial infections does have the similar effect on neurodegeneration is not clearly known. Thus, the systemic inflammation induced by bacterial infections has to be addressed, particularly within the experimental models of MS.

Role of TLRs in infection and EAE have been studied which are expressed on several types of cells, including peripheral lymphocytes, microglia cells and neurons (Kawai and Akira, 2007). Extracellular leucine-rich repeat domains of these receptors have been shown to be involved in specific pathogen recognition (Akira et al., 2006). However, the cytoplasmic domain is involved in signaling pathways. Upon sensing ligands, recruitment of adaptor proteins (MyD88 except for TLR3) takes place which in turns initiate the signal transduction pathway and leading to activation of NF-KB and induced the production of pro-inflammatory cytokines like TNF- $\alpha$, IL-6, and I L-1b etc. It is also observed that MyD88 pathway plays a crucial role in CNS infection and optic nerve injury (Zheng et al., 2012). In the previous study it has been shown that Chlamydia pneumoniae infection exacerbates EAE in mice. Moreover, from the mechanistic point of view Herrmann and coworkers showed that Streptococcus pneumoniae, a gram negative bacterium causes respiratory tract infection, can aggravate EAE via TLR2 without affecting the level of pro-inflammatory cytokines and T-cell proliferation. However, in both of these studies there was no evidence for the neuronal cell loss. These animal studies indicate the role of systemic infection on CNS, but whether these infections lead to the more pronounced neurodegeneration is not clearly known. In this study, we address the impact of infection on neuronal damage after the infection in acute as well as in chronic Infectious conditions. In our study despite of presence of $E$. coli in the peripheral blood circulation $24 \mathrm{~h}$ after the infection we did not observe any significant difference in the loss of RGCs between the animals infected with $E$. coli and the control during clinical and preclinical phase of disease. However, the loss of RGCs showed a trend for more pronounced RGCs loss of in the animals infected on day 1 EAE compare to its control. Thus, acute $E$. coli infection could possibly have long-term consequences in regards to neuronal degeneration.

We know from previous study that neuro-inflammation caused by external stimulus, which leads to the activation of microglial cells is not always deleterious 
(Glezer et al., 2006; Simard et al., 2006). However, in our study with the chronic infection model we infected the animals with $S$. aureus during the preclinical phase of disease, which induces a strong inflammatory response in the periphery showed by increased leukocyte counts. Surprisingly, we observed an increased neuronal survival in the animals infected with $S$. aureus compared to its control. These results indicated that the importance of BBB, that probably does not allow the inflammatory cells to enter into the CNS and induce the inflammatory signal across it. Moreover, by introducing Eap mutant strain in this study, we observed a higher neuronal survival in animals infected with wild-type bacterium compared to the animals infected with bacterium deficient for Eap. In our previous EAE studies with the same animal model, we observed that strong anti-inflammatory treatment is not always sufficient to prevent neurodegeneration (Diem et al., 2003; Maier et al., 2006; Rau et al., 2011). Even though we see a more neuronal survival in the animals infected with wild-type bacterium compared to the animals infected with bacterium deficient for Eap we cannot rule out a direct neuroprotective effect of Eap. Further studies are needed to clarify this issue. Nevertheless, by suppression of LFA-1 and ICAM-1 mediated transendothelial migration of immune cells into the CNS with consecutive neuroprotection, Eap has potential to serve as a new therapeutic for the treatment of MS.

\section{3: A clear distinction between the animal model of MS is important}

In the previous experimental studies it has been shown that the systemic inflammation induced by bacterial components lead to exacerbation of the disease course in EAE (Moreno et al., 2011; Schiffenbauer et al., 1993; Soos et al., 1995). Although in our studies we found that the inflammatory stimulus induce by a viable bacterium was more pronounced, it does not seem to have the similar effect as found in the previous finding performed by the use of solely bacterial components. Moreover, data from the acute infection model induced by $E$. coli also differed from the results obtained from the chronic infection model induced by the $S$. aureus. Nevertheless, the time point of infection as shown by Herrmann et al could also play determining role in the disease pathogenesis. From the previous and our current finding we conclude that the robust, but short lasting defined inflammatory stimulation induced by the bacterial components 
like LPS or enterotoxins felt adequately to reproduce the full spectrum of inflammatory changes a real systemic infection (Remick and Ward, 2005). Therefore, this is important to draw a clear distinction between these models of systemic inflammation to study various autoimmune diseases like MS. 


\section{6: CONCLUSIONS}

To address the question how does the bacterial infections (acute and chronic) influence the severity of disease in an experimental model of MS, we investigated the extent to which an acute infection with $E$. coli and chronic infection with $S$. aureus influences the disease course of MOG induced EAE in female Brown Norway rats. Moreover, we also investigated the effect of the acute and chronic bacterial infection on neurodegeneration.

In the current study, viable $E$. coli infection in female $\mathrm{BN}$ rats did not influence the clinical course of disease in EAE. Moreover, we did not observe any significant histopathological changes, performed in the optic nerve $s$ as well as on the survival of RGCs, in the group infected with E. coli compared to the control. Findings from these studies indicated that the systemic insult with bacterial components (Lipopolysaccharides, superantigens) do not reflect the true inflammatory conditions induced by a viable bacterial infection. Therefore this is important to draw a clear distinction between these two models of systemic inflammation.

In contrast to the acute infection, chronic infection induced by inoculating $S$. aureus into the agar of the implanted tissue cage showed a chronic systemic inflammatory response with increased in T- and B-cell counts as well as systemic production of pro-inflammatory cytokines. Unexpectedly, the chronic infection with $S$. aureus prevented the development of clinical EAE and markedly decreased the extent of inflammatory infiltrates and demyelination of the optic nerve. Moreover, using a $S$. aureus strain deficient for the extracellular adherence protein we showed the beneficial effect of a chronic bacterial infection on disease modulating effect in EAE is due to the secretion of this protein. Furthermore, infection with $S$. aureus also increased the number of surviving RGCs and axonal counts that seems to be related to the antiinflammatory properties of Eap. Further studies are needed to clarify the direct neuroprotective effect of Eap. 


\section{7: REFERENCES}

Akira, S., S. Uematsu, and O. Takeuchi. 2006. Pathogen recognition and innate immunity. Cell 124:783-801.

Alonso, A., and M.A. Hernan. 2008. Temporal trends in the incidence of multiple sclerosis: a systematic review. Neurology 71:129-135.

Andersen, O., P.E. Lygner, and et al. 1993. Viral infections trigger multiple sclerosis relapses: a prospective seroepidemiological study. J Neurol 240:417-422.

Andersson, P.B., E. Waubant, and et al. 1999. Multiple sclerosis that is progressive from the time of onset: clinical characteristics and progression of disability. Arch Neurol 56:11381142.

Archelos, J.J., S. Jung, and et al. 1993. Inhibition of experimental autoimmune encephalomyelitis by an antibody to the intercellular adhesion molecule ICAM-1. Ann Neurol 34:145-154.

Ascherio, A., and K.L. Munger. 2007. Environmental risk factors for multiple sclerosis. Part I: the role of infection. Ann Neurol 61:288-299.

Athanasopoulos, A.N., M. Economopoulou, and et al. 2006. The extracellular adherence protein (Eap) of Staphylococcus aureus inhibits wound healing by interfering with host defense and repair mechanisms. Blood 107:2720-2727.

Bach, J.F. 2002. The effect of infections on susceptibility to autoimmune and allergic diseases. N Engl J Med 347:911-920.

Bach, J.F. 2005. Infections and autoimmune diseases. J Autoimmun 25 Suppl:74-80.

Banks, W.A. 2005. Blood-brain barrier transport of cytokines: a mechanism for neuropathology. Curr Pharm Des 11:973-984.

Banks, W.A., L. Ortiz, and et al. 1991. Human interleukin (IL) 1 alpha, murine IL-1 alpha and murine IL-1 beta are transported from blood to brain in the mouse by a shared saturable mechanism. J Pharmacol Exp Ther 259:988-996.

Barcellos, L.F., S. Sawcer, and et al. 2006. Heterogeneity at the HLA-DRB1 locus and risk for multiple sclerosis. Hum Mol Genet 15:2813-2824.

Baxter, A.G. 2007. The origin and application of experimental autoimmune encephalomyelitis. Nat Rev Immunol 7:904-912.

Ben-Nun, A., I. Mendel, and et al. 1995. A 12-kDa protein of Mycobacterium tuberculosis protects mice against experimental autoimmune encephalomyelitis. Protection in the 
absence of shared T cell epitopes with encephalitogenic proteins. J Immunol 154:29392948.

Ben-Nun, A., and S. Yossefi. 1992. Staphylococcal enterotoxin B as a potent suppressant of $T$ Iymphocytes: trace levels suppress T lymphocyte proliferative responses. Eur J Immunol 22:1495-1503.

Bharadwaj, A.S., L.P. Schewitz-Bowers, and et al. 2013. Intercellular adhesion molecule 1 mediates migration of Th1 and Th17 cells across human retinal vascular endothelium. Invest Ophthalmol Vis Sci 54:6917-6925.

Bitsch, A., and W. Bruck. 2002. Differentiation of multiple sclerosis subtypes: implications for treatment. CNS Drugs 16:405-418.

Bloomgren, G., S. Richman, and et al. 2012. Risk of natalizumab-associated progressive multifocal leukoencephalopathy. N Engl J Med 366:1870-1880.

Bray, P.F., K.W. Culp, and et al. 1992. Demyelinating disease after neurologically complicated primary Epstein-Barr virus infection. Neurology 42:278-282.

Buljevac, D., H.Z. Flach, and et al. 2002. Prospective study on the relationship between infections and multiple sclerosis exacerbations. Brain 125:952-960.

Bullard, D.C., X. Hu, and et al. 2002. Prospective study on the relationship between infections and multiple sclerosis exacerbations. 2007. Intercellular adhesion molecule-1 expression is required on multiple cell types for the development of experimental autoimmune encephalomyelitis. J Immunol 178:851-857.

Cannella, B., A.H. Cross, and C.S. Raine. 1990. Upregulation and coexpression of adhesion molecules correlate with relapsing autoimmune demyelination in the central nervous system. J Exp Med 172:1521-1524.

Cepok, S., D. Zhou, Buljevac, D, and et al. 2002. Prospective study on the relationship between infections and multiple sclerosis exacerbations. 2005. Identification of Epstein-Barr virus proteins as putative targets of the immune response in multiple sclerosis. J Clin Invest 115:1352-1360.

Challoner, P.B., K.T. Smith, and et al. 1995. Plaque-associated expression of human herpesvirus 6 in multiple sclerosis. Proc Natl Acad Sci U S A 92:7440-7444.

Chataway, J., A. Mander, N. and et al. 2001. Multiple sclerosis in sibling pairs: an analysis of 250 families. J Neurol Neurosurg Psychiatry 71:757-761.

Chavakis, T., M. Hussain, and et al. 2002. Staphylococcus aureus extracellular adherence protein serves as anti-inflammatory factor by inhibiting the recruitment of host leukocytes. Nat Med 8:687-693. 
Chavakis, T., K.T. Preissner, and M. Herrmann. 2007. The anti-inflammatory activities of Staphylococcus aureus. Trends Immunol 28:408-418.

Chavakis, T., K. Wiechmann, and et al. 2005. Staphylococcus aureus interactions with the endothelium: the role of bacterial "secretable expanded repertoire adhesive molecules" (SERAM) in disturbing host defense systems. Thromb Haemost 94:278-285.

Christen, U., and M.G. von Herrath. 2005. Infections and autoimmunity--good or bad? J Immunol 174:7481-7486.

Clifford, D.B., A. De Luca, and et al. 2010. Natalizumab-associated progressive multifocal leukoencephalopathy in patients with multiple sclerosis: lessons from 28 cases. Lancet Neurol 9:438-446.

Compston, A. Lassmann, H, and McDonald, I. (1998). The story of multiple sclerosis. McAlpine‘s Multiple Sclerosis. 3rd ed. London: Churchill Livingstone.

Compston, A., and A. Coles. 2008. Multiple sclerosis. Lancet 372:1502-1517.

Correale, J., and M.F. Farez. 2011. The impact of environmental infections (parasites) on MS activity. Mult Scler 17:1162-1169.

Correale, J., and M.F. Farez. 2013. Parasite infections in multiple sclerosis modulate immune responses through a retinoic acid-dependent pathway. J Immunol 191:3827-3837.

Correale, J., M. Fiol, and W. Gilmore. 2006. The risk of relapses in multiple sclerosis during systemic infections. Neurology 67:652-659.

Cree, B.A. 2014. 2014 multiple sclerosis therapeutic update. Neurohospitalist 4:63-65.

Cunningham, C. 2013. Microglia and neurodegeneration: the role of systemic inflammation. Glia 61:71-90.

Damotte, V., L. Guillot-Noel, and et al. 2014. A gene pathway analysis highlights the role of cellular adhesion molecules in multiple sclerosis susceptibility. Genes Immun 15:126132.

Dhib-Jalbut, S., and S. Marks. 2010. Interferon-beta mechanisms of action in multiple sclerosis. Neurology 74 Suppl 1:S17-24.

Diem, R., M. Hobom, and et al. 2003. Methylprednisolone increases neuronal apoptosis during autoimmune CNS inflammation by inhibition of an endogenous neuroprotective pathway. J Neurosci 23:6993-7000.

Dinarello, C.A. 2000. Proinflammatory cytokines. Chest 118:503-508.

Du, C., S.Y. Yao, and et al. 2002. Chlamydia pneumoniae infection of the central nervous system worsens experimental allergic encephalitis. J Exp Med 196:1639-1644. 
Dyment, D.A., A.D. Sadovnick, and G.C. Ebers. 1997. Genetics of multiple sclerosis. Hum Mol Genet 6:1693-1698.

Ebers, G.C., D.E. Bulman, and et al. 1986. A population-based study of multiple sclerosis in twins. N Engl J Med 315:1638-1642.

Edwards, A.M., M.G. Bowden, and et al. 2012. Staphylococcus aureus extracellular adherence protein triggers TNFalpha release, promoting attachment to endothelial cells via protein A. PLoS One 7:e43046.

Edwards, S., M. Zvartau, and et al. 1998. Clinical relapses and disease activity on magnetic resonance imaging associated with viral upper respiratory tract infections in multiple sclerosis. J Neurol Neurosurg Psychiatry 64:736-741.

Engelhardt, B., and H. Wolburg. 2004. Mini-review: Transendothelial migration of leukocytes: through the front door or around the side of the house? Eur J Immunol 34:2955-2963.

Fleming, J.O., and T.D. Cook. 2006. Multiple sclerosis and the hygiene hypothesis. Neurology 67:2085-2086.

Ferrari, C.C., M.C. Pott Godoy, and et al. 2006. Progressive neurodegeneration and motor disabilities induced by chronic expression of IL-1beta in the substantia nigra. Neurobiol Dis 24:183-193.

Fitzner, D., and M. Simons. 2010. Chronic progressive multiple sclerosis - pathogenesis of neurodegeneration and therapeutic strategies. Curr Neuropharmacol 8:305-315.

Fleming, J.O., and T.D. Cook. 2006. Multiple sclerosis and the hygiene hypothesis. Neurology 67:2085-2086.

Foster, T.J., and M. Hook. 1998. Surface protein adhesins of Staphylococcus aureus. Trends Microbiol 6:484-488.

Fraser, J.D., and T. Proft. 2008. The bacterial superantigen and superantigen-like proteins. Immunol Rev 225:226-243.

Frohman, E.M., M.K. Racke, and C.S. Raine. 2006. Multiple sclerosis--the plaque and its pathogenesis. N Engl J Med 354:942-955.

Furlan, R., E. Brambilla, and et al. 2001. Intrathecal delivery of IFN-gamma protects C57BL/6 mice from chronic-progressive experimental autoimmune encephalomyelitis by increasing apoptosis of central nervous system-infiltrating lymphocytes. J Immunol 167:1821-1829.

Gale, C.R., and C.N. Martyn. 1995. Migrant studies in multiple sclerosis. Prog Neurobiol 47:425448. 
Gardinier, M.V., P. Amiguet, and et al. 1992. Myelin/oligodendrocyte glycoprotein is a unique member of the immunoglobulin superfamily. J Neurosci Res 33:177-187.

Ge, Y. (2006). Multiple sclerosis: the role of MR imaging. American Journal of Neuroradiology, 27(6), 1165-1176.

Genc, K., D.L. Dona, and A.T. Reder. 1997. Increased CD80(+) B cells in active multiple sclerosis and reversal by interferon beta-1b therapy. J Clin Invest 99:2664-2671.

Glezer, I., A. Lapointe, and S. Rivest. 2006. Innate immunity triggers oligodendrocyte progenitor reactivity and confines damages to brain injuries. FASEB J 20:750-752.

Gold, R., C. Linington, and H. Lassmann. 2006. Understanding pathogenesis and therapy of multiple sclerosis via animal models: 70 years of merits and culprits in experimental autoimmune encephalomyelitis research. Brain 129:1953-1971.

Gold, R., J.S. Wolinsky, and et al. 2010. Evolving expectations around early management of multiple sclerosis. Ther Adv Neurol Disord 3:351-367.

Gordon, E.J., K.J. Myers, and et al. 1995. Both anti-CD11a (LFA-1) and anti-CD11b (MAC-1) therapy delay the onset and diminish the severity of experimental autoimmune encephalomyelitis. J Neuroimmunol 62:153-160.

Goverman, J., A. Woods, and et al. 1993. Transgenic mice that express a myelin basic proteinspecific T cell receptor develop spontaneous autoimmunity. Cell 72:551-560.

Grady, M., and J.J. Cullen. 2003. Preventing postoperative Staphylococcus infections: an update. Surg Technol Int 11:57-60.

Haggar, A., C. Ehrnfelt, and et al. 2004. The extracellular adherence protein from Staphylococcus aureus inhibits neutrophil binding to endothelial cells. Infect Immun 72:6164-6167.

Haggar, A., J.I. Flock, and A. Norrby-Teglund. 2010. Extracellular adherence protein (Eap) from Staphylococcus aureus does not function as a superantigen. Clin Microbiol Infect 16:1155-1158.

Hallal-Longo, D.E., S.R. Mirandola, and et al. 2007. Diminished myelin-specific T cell activation associated with increase in CTLA4 and Fas molecules in multiple sclerosis patients treated with IFN-beta. J Interferon Cytokine Res 27:865-873.

Hamada, T., B.F. Driscoll, and et al. 1989. LPS augments adoptive transfer of experimental allergic encephalomyelitis in the Lewis rat. Autoimmunity 2:275-284.

Handel, A.E., G. Giovannoni, and et al. 2010. Environmental factors and their timing in adultonset multiple sclerosis. Nat Rev Neurol 6:156-166. 
Harraghy, N., M. Hussain, and et al. 2003. The adhesive and immunomodulating properties of the multifunctional Staphylococcus aureus protein Eap. Microbiology 149:2701-2707.

Herrmann, I., M. Kellert, and et al. 2006. Streptococcus pneumoniae Infection aggravates experimental autoimmune encephalomyelitis via Toll-like receptor 2. Infect Immun 74:4841-4848.

Hirotani, M., M. Niino, and H. Sasaki. 2010. The role of B cells in multiple sclerosis: implications for B-cell-targeted therapy. Curr Med Chem 17:3215-3222.

Hobom, M., M.K. Storch, and et al. 2004. Mechanisms and time course of neuronal degeneration in experimental autoimmune encephalomyelitis. Brain Pathol 14:148-157.

Hussain, M., Becker, K, and et al. (2001). Analogs of Eap protein are conserved and prevalent in clinical Staphylococcus aureus Isolates. Clin. Diagn. Lab. Immunol. 8, 1271-1276.

Jacobs, L.D., D.L. Cookfair, and et al. 1996. Intramuscular interferon beta-1a for disease progression in relapsing multiple sclerosis. The Multiple Sclerosis Collaborative Research Group (MSCRG). Ann Neurol 39:285-294.

Jacobs, L.D., D.L. Cookfair, and et al. 1995. A phase III trial of intramuscular recombinant interferon beta as treatment for exacerbating-remitting multiple sclerosis: design and conduct of study and baseline characteristics of patients. Multiple Sclerosis Collaborative Research Group (MSCRG). Mult Scler 1:118-135.

Janeway, C.A., Jr., J. Yagi, and et al. 1989. T-cell responses to Mls and to bacterial proteins that mimic its behavior. Immunol Rev 107:61-88.

Jaquiery, E., S. Jilek, and et al. 2010. Intrathecal immune responses to EBV in early MS. Eur J Immunol 40:878-887.

Jiang, H., R. Milo, P. and et al. 1995. Interferon beta-1b reduces interferon gamma-induced antigen-presenting capacity of human glial and B cells. J Neuroimmunol 61:17-25.

Johnson, K.P., B.R. Brooks, and et al. 1995. Copolymer 1 reduces relapse rate and improves disability in relapsing-remitting multiple sclerosis: results of a phase III multicenter, double-blind placebo-controlled trial. The Copolymer 1 Multiple Sclerosis Study Group. Neurology 45:1268-1276.

Kalkers, N.F., H. Vrenken, and et al. 2002. Brain atrophy in multiple sclerosis: impact of lesions and of damage of whole brain tissue. Mult Scler 8:410-414.

Kaneider, N.C., A.J. Leger, and A. Kuliopulos. 2006. Therapeutic targeting of molecules involved in leukocyte-endothelial cell interactions. FEBS J 273:4416-4424.

Kappos, L., D. Bates, and et al. 2011. Natalizumab treatment for multiple sclerosis: updated recommendations for patient selection and monitoring. Lancet Neurol 10:745-758. 
Kappos, L., E.W. Radue, and et al. 2010. A placebo-controlled trial of oral fingolimod in relapsing multiple sclerosis. $N$ Engl J Med 362:387-401.

Kawai, T., and S. Akira. 2007. Signaling to NF-kappaB by Toll-like receptors. Trends Mol Med 13:460-469.

Kim, J.S., K.S. Lee, and et al. 2000. Detection of human herpesvirus 6 variant $A$ in peripheral blood mononuclear cells from multiple sclerosis patients. Eur Neurol 43:170-173.

Komiyama, Y., S. Nakae, and et al. 2006. IL-17 plays an important role in the development of experimental autoimmune encephalomyelitis. J Immunol 177:566-573.

Kornek, B., and H. Lassmann. 1999. Axonal pathology in multiple sclerosis. A historical note. Brain Pathol 9:651-656.

Koutsis, G., M.E. Evangelopoulos, and et al. 2010. The onset of multiple sclerosis in Greece: a single-center study of 1,034 consecutive patients. Eur Neurol 63:350-356.

Kriesel, J.D., A. White, and et al. 2004. Multiple sclerosis attacks are associated with picornavirus infections. Mult Scler 10:145-148.

Kroepfl, J.F., L.R. Viise, and et al. 1996. Investigation of myelin/oligodendrocyte glycoprotein membrane topology. J Neurochem 67:2219-2222.

Kuhlmann, T., G. Lingfeld, and et al. 2002. Acute axonal damage in multiple sclerosis is most extensive in early disease stages and decreases over time. Brain 125:2202-2212.

Kurtzke, J.F. 2005. Epidemiology and etiology of multiple sclerosis. Phys Med Rehabil Clin $N$ Am 16:327-349.

Kutzelnigg, A., and H. Lassmann. 2005. Cortical lesions and brain atrophy in MS. J Neurol Sci 233:55-59.

La Flamme, A.C., K. Ruddenklau, and B.T. Backstrom. 2003. Schistosomiasis decreases central nervous system inflammation and alters the progression of experimental autoimmune encephalomyelitis. Infect Immun 71:4996-5004.

Langford, M.P., G.J. Stanton, and H.M. Johnson. 1978. Biological effects of staphylococcal enterotoxin A on human peripheral lymphocytes. Infect Immun 22:62-68.

Langrish, C.L., Y. Chen, and et al. 2005. IL-23 drives a pathogenic T cell population that induces autoimmune inflammation. J Exp Med 201:233-240.

Laschinger, M., and B. Engelhardt. 2000. Interaction of alpha4-integrin with VCAM-1 is involved in adhesion of encephalitogenic $\mathrm{T}$ cell blasts to brain endothelium but not in their transendothelial migration in vitro. J Neuroimmunol 102:32-43.

Lee, B.P., and B.A. Imhof. 2008. Lymphocyte transmigration in the brain: a new way of thinking. Nat Immunol 9:117-118. 
Lee, L.Y., Y.J. Miyamoto, B.W. McIntyre, M. Hook, K.W. McCrea, D. McDevitt, and E.L. Brown. 2002. The Staphylococcus aureus Map protein is an immunomodulator that interferes with T cell-mediated responses. J Clin Invest 110:1461-1471.

Leibowitz, U., and M. Alter. 1970. Clinical factors associated with increased disability in multiple sclerosis. Acta Neurol Scand 46:53-70.

Leibowitz, U., A. Antonovsky, and et al. 1966. Epidemiological study of multiple sclerosis in Israel. II. Multiple sclerosis and level of sanitation. J Neurol Neurosurg Psychiatry 29:6068.

Lucet, J.C., M. Herrmann, P. and et al. 1990. Treatment of experimental foreign body infection caused by methicillin-resistant Staphylococcus aureus. Antimicrob Agents Chemother 34:2312-2317.

Maier, K., A.V. Kuhnert, N. and et al. Diem. 2006. Effects of glatiramer acetate and interferonbeta on neurodegeneration in a model of multiple sclerosis: a comparative study. Am J Pathol 169:1353-1364.

Mancuso, R., S. Delbue, and et al. 2007. Increased prevalence of varicella zoster virus DNA in cerebrospinal fluid from patients with multiple sclerosis. J Med Virol 79:192-199.

Markovic-Plese, S., C. Pinilla, and R. Martin. 2004. The initiation of the autoimmune response in multiple sclerosis. Clin Neurol Neurosurg 106:218-222.

McElroy, J.P., and J.R. Oksenberg. 2011. Multiple sclerosis genetics 2010. Neurol Clin 29:219231.

McFarland, H.F., and R. Martin. 2007. Multiple sclerosis: a complicated picture of autoimmunity. Nat Immunol 8:913-919.

McGeer, E.G., A. Klegeris, and P.L. McGeer. 2005. Inflammation, the complement system and the diseases of aging. Neurobiol Aging 26 Suppl 1:94-97.

McLeod, J.G., S.R. Hammond, and J.F. Kurtzke. 2012. Migration and multiple sclerosis in United Kingdom and Ireland immigrants to Australia: a reassessment. II. Characteristics of early (pre-1947) compared to later migrants. J Neurol 259:684-693.

Melzer, N., S.G. Meuth, and et al. 2008. A beta-lactam antibiotic dampens excitotoxic inflammatory CNS damage in a mouse model of multiple sclerosis. PLoS One 3:e3149.

Metz, I., S.D. Weigand, and et al. 2014. Pathologic heterogeneity persists in early active multiple sclerosis lesions. Ann Neurol 75:728-738.

Meyer, R., R. Weissert, and et al. 2001. Acute neuronal apoptosis in a rat model of multiple sclerosis. J Neurosci 21:6214-6220. 
Miller, D.H., S.R. Hammond, and et al. 1990. Multiple sclerosis in Australia and New Zealand: are the determinants genetic or environmental? J Neurol Neurosurg Psychiatry 53:903905.

Miller, D.H., and S.M. Leary. 2007. Primary-progressive multiple sclerosis. Lancet Neurol 6:903912.

Miller, D.H., D. Soon, and et al. 2007. MRI outcomes in a placebo-controlled trial of natalizumab in relapsing MS. Neurology 68:1390-1401.

Mor, F., and I.R. Cohen. 2013. Beta-lactam antibiotics modulate T-cell functions and gene expression via covalent binding to cellular albumin. Proc Natl Acad Sci U S A 110:29812986.

Moreno, B., J.P. Jukes, and et al. 2011. Systemic inflammation induces axon injury during brain inflammation. Ann Neurol 70:932-942.

MS atlas., 2013, http://www.atlasofms.org/

Munoz-Culla, M., H. Irizar, and D. Otaegui. 2013. The genetics of multiple sclerosis: review of current and emerging candidates. App/ Clin Genet 6:63-73.

Murillo, O., A. Domenech, A. and et al. 2006. Efficacy of high doses of levofloxacin in experimental foreign-body infection by methicillin-susceptible Staphylococcus aureus. Antimicrob Agents Chemother 50:4011-4017.

Murphy, C.A., R.M. Hoek, and et al. 2002. Interactions between hemopoietically derived TNF and central nervous system-resident glial chemokines underlie initiation of autoimmune inflammation in the brain. J Immunol 169:7054-7062.

National MS Society, http://www.nationalmssociety.org/

Nguyen, M.D., T. D'Aigle, and et al. 2004. Exacerbation of motor neuron disease by chronic stimulation of innate immunity in a mouse model of amyotrophic lateral sclerosis. $J$ Neurosci 24:1340-1349.

Nguyen, M.D., W.E. Mushynski, and J.P. Julien. 2002. Cycling at the interface between neurodevelopment and neurodegeneration. Cell Death Differ 9:1294-1306.

Nogai, A., V. Siffrin, and et al. 2005. Lipopolysaccharide injection induces relapses of experimental autoimmune encephalomyelitis in nontransgenic mice via bystander activation of autoreactive CD4+ cells. J Immunol 175:959-966.

Opsahl, M.L., and P.G. Kennedy. 2005. Early and late HHV-6 gene transcripts in multiple sclerosis lesions and normal appearing white matter. Brain 128:516-527.

Ordonez, G., B. Pineda, and et al. 2004. Brief presence of varicella-zoster vral DNA in mononuclear cells during relapses of multiple sclerosis. Arch Neurol 61:529-532. 
Orton, S.M., S.V. Ramagopalan, and et al. 2010. Effect of immigration on multiple sclerosis sex ratio in Canada: the Canadian Collaborative Study. J Neurol Neurosurg Psychiatry 81:31-36.

Panitch, H.S. 1994. Influence of infection on exacerbations of multiple sclerosis. Ann Neurol 36 Suppl:S25-28.

Patsopoulos, N.A., F. Esposito, and et al. 2011. Genome-wide meta-analysis identifies novel multiple sclerosis susceptibility loci. Ann Neurol 70:897-912.

Peavy, D.L., W.H. Adler, and R.T. Smith. 1970. The mitogenic effects of endotoxin and staphylococcal enterotoxin $B$ on mouse spleen cells and human peripheral lymphocytes. J Immunol 105:1453-1458.

Pender, M.P. 2011. The essential role of Epstein-Barr virus in the pathogenesis of multiple sclerosis. Neuroscientist 17:351-367.

Perry, V.H. 2010. Contribution of systemic inflammation to chronic neurodegeneration. Acta Neuropathol 120:277-286.

Perry, V.H., C. Cunningham, and C. Holmes. 2007. Systemic infections and inflammation affect chronic neurodegeneration. Nat Rev Immunol 7:161-167.

Polazzi, E., and A. Contestabile. 2002. Reciprocal interactions between microglia and neurons: from survival to neuropathology. Rev Neurosci 13:221-242.

Polman, C.H., P.W. O'Connor, and et al. 2006. A randomized, placebo-controlled trial of natalizumab for relapsing multiple sclerosis. N Engl J Med 354:899-910.

Polman, C.H., S.C. Reingold, and et al. 2011. Diagnostic criteria for multiple sclerosis: 2010 revisions to the McDonald criteria. Ann Neurol 69:292-302.

Popescu, B.F., and C.F. Lucchinetti. 2012. Pathology of demyelinating diseases. Annu Rev Pathol 7:185-217.

Poser, C.M., D.W. Paty, and et al. 1983. New diagnostic criteria for multiple sclerosis: guidelines for research protocols. Ann Neurol 13:227-231.

Rand, K.H., H. Houck, and et al. 2000. Epstein-Barr virus nuclear antigen-1 (EBNA-1) associated oligoclonal bands in patients with multiple sclerosis. J Neurol Sci 173:32-39.

Rau, C.R., K. Hein, and et al. 2011. Anti-inflammatory effects of FTY720 do not prevent neuronal cell loss in a rat model of optic neuritis. Am J Pathol 178:1770-1781.

Remick, D.G., and P.A. Ward. 2005. Evaluation of endotoxin models for the study of sepsis. Shock 24 Suppl 1:7-11.

Rezaali, S., A. Khalilnezhad, and et al. 2013. Epidemiology of multiple sclerosis in Qom: Demographic study in Iran. Iran J Neurol 12:136-143. 
Rosenberg, G.A. 2012. Neurological diseases in relation to the blood-brain barrier. J Cereb Blood Flow Metab 32:1139-1151.

Ross, J., K. Schumacher, and E. Mangete. 1965. The demonstration and identification of a factor of the nature of immune globulin $M$ and the antibody properties in the serum of patients with multiple sclerosis. Klin Wochenschr 43:1324-1331.

Rossi, B., S. Angiari, and et al. 2011. Vascular inflammation in central nervous system diseases: adhesion receptors controlling leukocyte-endothelial interactions. J Leukoc Biol 89:539-556.

Rothhammer, V., S. Heink, F. and et al. 2011. Th17 lymphocytes traffic to the central nervous system independently of alpha4 integrin expression during EAE. J Exp Med 208:24652476.

Rovaris, M., C. Confavreux, and et al. 2006. Secondary progressive multiple sclerosis: current knowledge and future challenges. Lancet Neurol 5:343-354.

Ruddle, N.H., C.M. Bergman, and et al. 1990. An antibody to lymphotoxin and tumor necrosis factor prevents transfer of experimental allergic encephalomyelitis. J Exp Med 172:11931200.

Runmarker, B., and O. Andersen. 1993. Prognostic factors in a multiple sclerosis incidence cohort with twenty-five years of follow-up. Brain 116 ( Pt 1):117-134.

Sadovnick, A.D. 1993. Familial recurrence risks and inheritance of multiple sclerosis. Curr Opin Neurol Neurosurg 6:189-194.

Sadovnick, A.D., I.M. Yee, and et al. 2009. Age of onset in concordant twins and other relative pairs with multiple sclerosis. Am J Epidemiol 170:289-296.

Samoilova, E.B., J.L. Horton, and et al. 1998. IL-6-deficient mice are resistant to experimental autoimmune encephalomyelitis: roles of IL-6 in the activation and differentiation of autoreactive T cells. J Immunol 161:6480-6486.

Sawcer, S., G. Hellenthal, and et al. 2011. Genetic risk and a primary role for cell-mediated immune mechanisms in multiple sclerosis. Nature 476:214-219.

Schiffenbauer, J., H.M. Johnson, and et al. 1993. Staphylococcal enterotoxins can reactivate experimental allergic encephalomyelitis. Proc Natl Acad Sci U S A 90:8543-8546.

Schneider, K.M. 2005. AANA Journal course: update for nurse anesthetists--an overview of multiple sclerosis and implications for anesthesia. AANA J 73:217-224.

Schrijver, I.A., M. van Meurs, and et al. 2001. Bacterial peptidoglycan and immune reactivity in the central nervous system in multiple sclerosis. Brain 124:1544-1554. 
Scriba, T.J., S. Sierro, and et al. 2008. The Staphyloccous aureus Eap protein activates expression of proinflammatory cytokines. Infect Immun 76:2164-2168.

Shapira, L., S. Ayalon, and T. Brenner. 2002. Effects of Porphyromonas gingivalis on the central nervous system: activation of glial cells and exacerbation of experimental autoimmune encephalomyelitis. J Periodontol 73:511-516.

Sibley, W.A., C.R. Bamford, and K. Clark. 1985. Clinical viral infections and multiple sclerosis. Lancet 1:1313-1315.

Sibley, W.A., and J.M. Foley. 1965. INFECTION AND IMMUNIZATION IN MULTIPLE SCLEROSIS. Ann N Y Acad Sci 122:457-466.

Simard, A.R., D. Soulet, and et al. 2006. Bone marrow-derived microglia play a critical role in restricting senile plaque formation in Alzheimer's disease. Neuron 49:489-502.

Simpson, S., Jr., L. Blizzard, and et al. 2011. Latitude is significantly associated with the prevalence of multiple sclerosis: a meta-analysis. J Neurol Neurosurg Psychiatry 82:1132-1141.

Soos, J.M., A.C. Hobeika, E.J. and et al. 1995. Accelerated induction of experimental allergic encephalomyelitis in PL/J mice by a non-V beta 8-specific superantigen. Proc Natl Acad Sci U S A 92:6082-6086.

Sospedra, M., Y. Zhao, and et al. 2005. Recognition of conserved amino acid motifs of common viruses and its role in autoimmunity. PLoS Pathog 1:e41.

Steiner, O., C. Coisne, and et al. 2010. Differential roles for endothelial ICAM-1, ICAM-2, and VCAM-1 in shear-resistant T cell arrest, polarization, and directed crawling on bloodbrain barrier endothelium. J Immunol 185:4846-4855.

Strachan, D.P. 1989. Hay fever, hygiene, and household size. BMJ 299:1259-1260.

Swanborg, R.H. 1995. Experimental autoimmune encephalomyelitis in rodents as a model for human demyelinating disease. Clin Immunol Immunopathol 77:4-13.

Tauber, S.C., R. Nau, and J. Gerber. 2007. Systemic infections in multiple sclerosis and experimental autoimmune encephalomyelitis. Arch Physiol Biochem 113:124-130.

Teleshova, N., W. Bao, and et al. 2000. Elevated CD40 ligand expressing blood T-cell levels in multiple sclerosis are reversed by interferon-beta treatment. Scand J Immunol 51:312320.

Trapp, B.D., J. Peterson, and et al. 1998. Axonal transection in the lesions of multiple sclerosis. N Engl J Med 338:278-285.

Trapp, B.D., R. Ransohoff, and R. Rudick. 1999. Axonal pathology in multiple sclerosis: relationship to neurologic disability. Curr Opin Neurol 12:295-302. 
Traugott, U., D.E. McFarlin, and C.S. Raine. 1986. Immunopathology of the lesion in chronic relapsing experimental autoimmune encephalomyelitis in the mouse. Cell Immunol 99:395-410.

Tsao, N., H.P. Hsu, and et al. 2001. Tumour necrosis factor-alpha causes an increase in bloodbrain barrier permeability during sepsis. J Med Microbiol 50:812-821.

van Oers, M.H., M. Van Glabbeke, and et al. 2010. Rituximab maintenance treatment of relapsed/resistant follicular non-Hodgkin's lymphoma: long-term outcome of the EORTC 20981 phase III randomized intergroup study. J Clin Oncol 28:2853-2858.

Visser, L., H. Jan de Heer, and et al. 2005. Proinflammatory bacterial peptidoglycan as a cofactor for the development of central nervous system autoimmune disease. $\mathrm{J}$ Immunol 174:808-816.

Wang, D., Z. Lu, and et al. 2009. Macrolide antibiotics aggravate experimental autoimmune encephalomyelitis and inhibit inducible nitric oxide synthase. Immunol Invest 38:602612.

Wang, H., J. von Rohrscheidt, and et al. 2010. Extracellular adherence protein of Staphylococcus aureus suppresses disease by inhibiting T-cell recruitment in a mouse model of psoriasis. J Invest Dermatol 130:743-754.

Warner, H.B., and R.I. Carp. 1981. Multiple sclerosis and Epstein-Barr virus. Lancet 2:1290.

Weinshenker, B.G. 1994. Natural history of multiple sclerosis. Ann Neurol 36 Suppl:S6-11.

Weinshenker, B.G., G.P. Rice, and et al. 1991. The natural history of multiple sclerosis: a geographically based study. 4. Applications to planning and interpretation of clinical therapeutic trials. Brain 114 ( Pt 2):1057-1067.

Weissert, R., E. Wallstrom, M.K. and et al. 1998. MHC haplotype-dependent regulation of MOGinduced EAE in rats. J Clin Invest 102:1265-1273.

Wekerle, H. 2008. Lessons from multiple sclerosis: models, concepts, observations. Ann Rheum Dis 67 Suppl 3:iii56-60.

Wekerle, H., K. Kojima, and et al. 1994. Animal models. Ann Neurol 36 Suppl:S47-53.

White, J., A. Herman, and et al. 1989. The $\vee$ beta-specific superantigen staphylococcal enterotoxin B: stimulation of mature $\mathrm{T}$ cells and clonal deletion in neonatal mice. Cell 56:27-35.

Willenborg, D.O., S. Fordham, and et al. 1996. IFN-gamma plays a critical down-regulatory role in the induction and effector phase of myelin oligodendrocyte glycoprotein-induced autoimmune encephalomyelitis. J Immunol 157:3223-3227. 
Wittchen, E.S. 2009. Endothelial signaling in paracellular and transcellular leukocyte transmigration. Front Biosci (Landmark Ed) 14:2522-2545.

Wolswijk, G. 1998. Chronic stage multiple sclerosis lesions contain a relatively quiescent population of oligodendrocyte precursor cells. J Neurosci 18:601-609.

Wucherpfennig, K.W., and J.L. Strominger. 1995. Molecular mimicry in T cell-mediated autoimmunity: viral peptides activate human $\mathrm{T}$ cell clones specific for myelin basic protein. Cell 80:695-705.

Xie, C., P. Alcaide, and et al. 2006. Suppression of experimental autoimmune encephalomyelitis by extracellular adherence protein of Staphylococcus aureus. J Exp Med 203:985-994.

Yednock, T.A., C. Cannon, and et al. 1992. Prevention of experimental autoimmune encephalomyelitis by antibodies against alpha 4 beta 1 integrin. Nature 356:63-66.

Zamvil, S.S., and L. Steinman. 1990. The T lymphocyte in experimental allergic encephalomyelitis. Annu Rev Immunol 8:579-621.

Zhang, H.L., M.Y. Hassan, and et al. 2012. Attenuated EAN in TNF-alpha deficient mice is associated with an altered balance of M1/M2 macrophages. PLoS One 7:e38157.

Zhang, J., S. Markovic-Plese, and et al. 1994. Increased frequency of interleukin 2-responsive T cells specific for myelin basic protein and proteolipid protein in peripheral blood and cerebrospinal fluid of patients with multiple sclerosis. J Exp Med 179:973-984.

Zheng, Z., R. Yuan, M. and et al. 2012. The toll-like receptor 4-mediated signaling pathway is activated following optic nerve injury in mice. Brain Res 1489:90-97.

Zhou, X., W. Hu, and X. Qin. 2008. The role of complement in the mechanism of action of rituximab for B-cell lymphoma: implications for therapy. Oncologist 13:954-966.

Zimmerli, W., F.A. Waldvogel, and et al. 1982. Pathogenesis of foreign body infection: description and characteristics of an animal model. J Infect Dis 146:487-497. 


\section{List of abbreviations}

${ }^{\circ} \mathrm{C}$

$\mu l$

APC

ATCC

BBB

BN

CD

CFU

CNS

CSF

Cy

DNA

E. coli

EAE

Eap

EBV

EDSS

EDTA

FACS

FG

FITC

FPRL-1

FTY

G

GA

HLA

HRP

$\mathrm{H}$
Degree Celsius

Microliter

Antigen-presenting cell

American Type Culture Collection

Blood brain barrier

Brown Norway

Cluster of differentiation

Colony forming units

Central nervous system

Cerebro spinal fluid

Cyanine

Deoxyribonucleic acid

Escherichia coli

Experimental autoimmune encephalomyelitis

Extracellular adherence protein

Epstein Barr virus

Expanded Disability Status Scale

Ethylenediaminetetraacetic acid

Fluorescence-activated cell sorting

Fluorogold

Fluorescein isothiocyanate

Formyl peptide receptor-like 1

Fingolimod

Gram

Glatiramer acetate

Human leukocyte antigen

Horseradish peroxidase

Hour 
ICAM-1

IFN

$\lg$

$\mathrm{IHC}$

IL

Ip

$\mathrm{Kg}$

L

LFA-1

LFB

LPS

MAG

MBP

$\mathrm{MHC}$

$\mathrm{Ml}$

$\mathrm{Mm}$

MOG

MOMP

MRI

MS

$\mathrm{MSCH}$

$\mathrm{Mt}$

$\mathrm{NaCl}$

ON

PBS

PE

PFA

Pi

PLP

PML

PMMA
Intercellular adhesion molecule-1

Interferon

Immunoglobulin

Immunohistochemistry

Interleukin

Intraperitoneal

Kilogram

Liter

Lymphocyte function-associated antigen-1

Luxol fast blue stain

Lipopolysaccharides

Myelin-associated glycoprotein

Myelin basic protein

Major histocompatibility complex

Milliliters

Millimeters

Myelin oligodendrocyte glycoprotein

Major outer membrane protein

Magnetic resonance imaging

Multiple sclerosis

Mouse spinal cord homogenate

Mycobacterium tuberculosis

Sodium chloride

Optic nerve

Phosphate buffered saline

Phycoerythrin

Paraformaldehyde

Post immunization

Proteolipid protein

Progressive Multifocal Leukoencephalopathy

Polymethylmethacrylate 


$\begin{array}{ll}\text { PMN } & \text { Polymorphonuclear leukocytes } \\ \text { PPMS } & \text { Primary progressive multiple sclerosis } \\ \text { PSGL-1 } & \text { P-selectin glycoprotein ligand-1 } \\ \text { RGC } & \text { Retinal ganglion cells } \\ \text { ROI } & \text { Regions of interest } \\ \text { RRMS } & \text { Relapsing-remitting multiple sclerosis } \\ \text { RT } & \text { Room temperature } \\ \text { S. aureus } & \text { Staphylococcus aureus } \\ \text { S1P } & \text { Sphingosine-1 phosphate } \\ \text { SERAM } & \text { Secretable expanded repertoire adhesive molecules } \\ \text { SPMS } & \text { Secondary progressive multiple sclerosis } \\ \text { TCF } & \text { Tissue cage fluid } \\ \text { TGF-b } & \text { Transforming growth factor beta } \\ \text { TLR } & \text { Toll-like receptors } \\ \text { TNF } & \text { Tumor necrosis factors } \\ \text { Vb } & \text { Variable region } \\ \text { VCAM-1 } & \text { Vascular cell adhesion molecule 1 } \\ \text { VLA-4 } & \text { Very late Intigen-4 } \\ & \end{array}$




\section{Acknowledgements}

This work was carried out at the department of Neurology, University Medicine Göttingen (UMG), Göttingen. Last few years, I spend in UMG Göttingen was incredible and I wish to convey my gratitude to a number of people who have assisted either directly or indirectly over the past four years in the completion of this thesis.

I would like to thank Prof. Mathias Bähr for giving me the opportunity to do my Ph.D. in his department. I would also like to thank him for his valuable suggestions, critical discussions and his supervision and it has been a great privilege to work and learn under his supervision.

No less, I would like to thank my co-supervisor Dr. Katharina Hein for excellent guidance throughout this project. Her encouragement, inspiration and emotional support were crucial during this thesis work.

I would like to thank Prof. Dr. Roland Nau (Institute of neuropathology, University Medicine Göttingen) for helping me in establishing the chronic infection model and his valuable and critical suggestions during this work. I also thank my doctoral committee members Prof. Dr. Mikael Simons and Prof. Dr. Klaus-Armin Nave for their useful suggestions and valuable feedback for my thesis.

I would also like to thank Else Kröner-Fresenius-Stiftung for funding this project and a partial support by Göttingen Graduate School for Neurosciences und Molecular Biosciences (DFG Grant GSC226/2) is also appreciated.

I would also like to thank my lab members Katharina Friebe, Sabine, Benedikt, Dorothea Birte and our lab technician Irina for maintaining a healthy working environment in the lab.

I wholeheartedly thank Satya, Somu, Saket, Akhil, Rikky, Manish, Madhav, Sadanand, Sanjeev, Vimal, Sai Aman and my child hood friend Bhaskar for a warm and really long withstanding friendship, which I will always cherish.

Last but not the least I am deeply grateful to my all the family members, especially my mother, and my love, Rashmi simply for being there and for patiently waiting for my calls.

Many thanks to all of you 


\section{Curriculum vitae}

\section{Personal details}

Email: - singh_prats31@yahoo.co.in

Address C/O:- Ravi Shankar Singh

At + Po: - Sarsi, Purnia, Bihar

Pin: - 854306

Date of Birth: - 26-10-1983

Nationality: - Indian

Gender: - Male

\section{Academic Qualifications}

Ph. D. Laboratory of Prof. Mathias Bähr, Department of Neurology, UMG, Goettingen, (January 2011-till date)

Master's (M.Sc.) in Biotechnology from School of Life Sciences, University of Hyderabad, India, (2008-2010)

Bachelors (B.Sc.) in biotechnology from Allahabad Agricultural Institute, Deemed University, Allahabad, India, (2004-2008)

\section{Poster presentations}

Prateek Kumar, Sven Wichert, Benedikt Kretzschmar, Mathias Bähr, Moritz Rossner, Katharina Hein, Gene expression analysis of retinal ganglion cells in experimental autoimmune optic neuritis. $10^{\text {th }}$ Göttingen, meeting of the German Neuroscience Society. March 13-16-2013, Göttingen, Germany.

Prateek Kumar, Benedikt Kretzschmar, Sabine Herold, Roland Nau, Doron Merkler, Sandra Schütze, Mathias Bähr, Katharina Hein, Impact of chronic Staphylococcus aureus infection on clinical course of disease and neurodegeneration in MOG-EAE. Microglia: Guardians of the Brain, EMBL Heidelberg, Germany 


\section{List of publications}

Kumar, P., B. Kretzschmar, S. Herold, R. Nau, M. Kreutzfeldt, S. Schütze, M. Bahr, and K. Hein. 2015. Beneficial effect of chronic Staphylococcus aureus infection in a model of multiple sclerosis is mediated through the secretion of extracellular adherence protein. $\mathrm{J}$ Neuroinflammation 12:22.

Sabine Herold ${ }^{\#}$, Prateek Kumar", Sven P. Wichert, Benedikt Kretzschmar' Mathias Bähr, Moritz J. Rossner, Katharina Hein Neurodegeneration in auto-immune optic neuritis is associated with altered APP cleavage in neurons and transcriptional upregulation of p53. Submitted (Under review)

Prateek Kumar, Catherine Friebe, Rieka Schallorn, Roland Nau, Sandra Schütze, Mathias Bähr, Katharina Hein, Acute Escherichia coli infection has no influence clinical course and the extent of neurodegeneration in animal model of multiple sclerosis. Submitted (Under review)

\# Equally contributed first authors 